Supporting Information (Part 1 of 2) for

\title{
Pentacene Oligomers and Polymers: Functionalization of Pentacene To Afford Mono-, Di-, Tri- and Polymeric Materials.
}

\author{
Dan Lehnherr and Rik R. Tykwinski* \\ Department of Chemistry, University of Alberta, Edmonton AB T6G 2G2, Canada
}

rik.tykwinski@ualberta.ca 


\section{Table of Contents For Supporting Information (Part 1 of 2)}

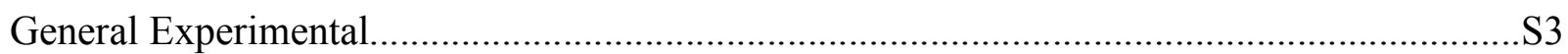

Synthetic Procedures and Spectral Characterization of Compounds 1-21 and S2................S4

Discussion of MALDI Mass Spectrometry Results and Structures of Polymers 8 and 9.........S25

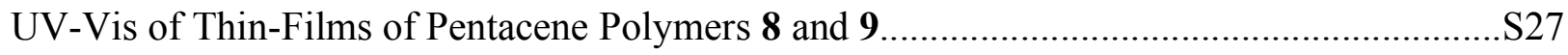

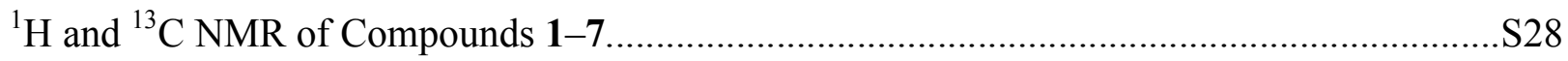




\section{General Experimental}

Reagents were purchased in reagent grade from commercial suppliers and used without further purification. 6,13-pentacenequinone ${ }^{1}$ was recrystallized from $N, N$-dimethylformamide and dried under vacuum. $\mathrm{Et}_{2} \mathrm{O}$ and THF were distilled from sodium/benzophenone ketyl; $\mathrm{CH}_{3} \mathrm{CN}$ and $\mathrm{CH}_{2} \mathrm{Cl}_{2}$ were distilled from $\mathrm{CaH}_{2}$. Anhydrous $\mathrm{MgSO}_{4}$ was used as the drying agent after aqueous work-up. Evaporation and concentration in vacuo was done at water-aspirator pressure. All reactions were performed in standard, dry glassware under an inert atmosphere of nitrogen. Column chromatography: silica gel-60 (230-400 mesh); size exclusion chromatography: BioRad Bio-Beads S-X2 (200-400 mesh). Thin Layer Chromatography (TLC): pre-coated plastic sheets covered with $0.20 \mathrm{~mm}$ silica gel with fluorescent indicator UV $254 \mathrm{~nm}$; visualization by UV light or $\mathrm{KMnO}_{4}$ stain. ${ }^{1} \mathrm{H}$ - and ${ }^{13} \mathrm{C}-\mathrm{NMR}$ spectra were collected at $27{ }^{\circ} \mathrm{C}$ in $\mathrm{CDCl}_{3}$; solvent peaks (7.24 for ${ }^{1} \mathrm{H}$ and 77.0 for $\left.{ }^{13} \mathrm{C}\right)$ as reference. Coupling constants are reported as observed $( \pm 0.5$ $\mathrm{Hz}$ ). For simplicity, the coupling constants of the aryl protons for para-substituted aryl groups have been reported as pseudo first-order (i.e., doublets), even though they are second-order (AA'BB') spin systems. UV-vis spectra acquired at rt using a Varian Cary 400 Scan Spectrometer; $\lambda_{\max }$ in $\mathrm{nm}\left(\varepsilon\right.$ in $\left.\mathrm{L} \cdot \mathrm{mol}^{-1} \cdot \mathrm{cm}^{-1}\right)$. Emission spectrum recorded using Photon Technology International (PTI) MP1 Fluorescence system. For mass spectral analyses, lowresolution data are provided in cases when $\mathrm{M}^{+}$is not the base peak; otherwise, only highresolution data are provided. Samples for ESI mass spectrometry were dissolved in a chlorinated solvent $\left(\mathrm{CHCl}_{3}, \mathrm{CH}_{2} \mathrm{Cl}_{2}\right.$ or $\left.\mathrm{ClCH}_{2} \mathrm{CH}_{2} \mathrm{Cl}_{2}\right)$ and made use of a 3:1 $\mathrm{MeOH}$ /toluene mixture as the carrier solvent. MALDI mass spectrometry used the matrix trans-2-[3-(4-tert-butylphenyl)-2methyl-2-propenylidene]malononitrile (DCTB).

Differential scanning calorimetries (DSC) were measured on a Perkin Elmer Pyris 1 DSC instrument. Thremogravometric analyses (TGA) were carried out on a Perkin Elmer Pyris 1 TGA instrument. All thermal analyses were carried out under a flow of nitrogen with a heating rate of $10^{\circ} \mathrm{C} / \mathrm{min}$ unless noted otherwise. Thermal decomposition temperature as measured by TGA (as sample weight loss) are reported as $\mathrm{T}_{\mathrm{d}}$ in which the temperature listed corresponds to the intersection of the tangent lines of the baseline and the edge of the peak corresponding to the first significant weight loss, typically $>5 \%$. Melting points from DSC are reported as a temperature range, except in cases when the sample decomposed, in which case the onset temperature of the decomposition exothermic peak are reported as well as the exothermic maxima corresponding to the decomposition. Experimental procedures for compounds synthesized are listed in numerical order.

${ }^{1}$ Reid, W.; Anthöfer, F. Angew. Chem. 1953, 65, 601. 


\section{Synthetic procedures and spectral characterization of compounds 1-21 and S2}

Synthesis of 1.

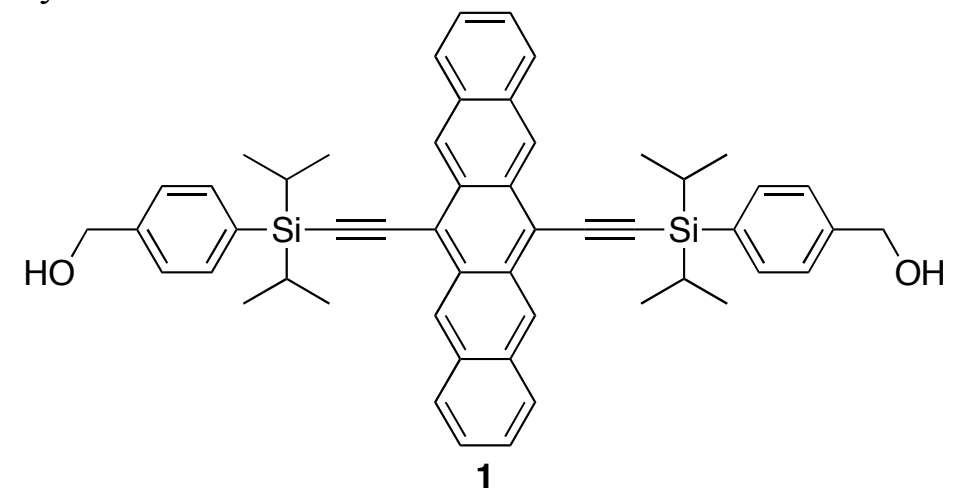

To a solution of $7(2.105 \mathrm{~g}, 2.114 \mathrm{mmol})$ in dry THF $(200 \mathrm{~mL})$ was added dropwise an aqueous solution of $\sim 1.17 \mathrm{M} \mathrm{HCl}(5.8 \mathrm{~mL}$; prepared by adding $10.0 \mathrm{~mL}$ of concentrated $\mathrm{HCl}(36 \%)$ to $90.0 \mathrm{~mL}$ of water). The solution was allowed to stir at $\mathrm{rt}$ for $6 \mathrm{~h}$ before adding $5 \%$ aq. $\mathrm{NaHCO}_{3}$ $(150 \mathrm{~mL})$ and $\mathrm{CH}_{2} \mathrm{Cl}_{2}(200 \mathrm{~mL})$. The mixture was separated and the organic phase was washed with $5 \%$ aq. $\mathrm{NaHCO}_{3}(150 \mathrm{~mL})$, satd. aq. $\mathrm{NaCl}(150 \mathrm{~mL})$, dried $\left(\mathrm{MgSO}_{4}\right)$, and the solvent removed in vacuo. Column chromatography (silica gel, 1:1 hexanes/ethyl acetate) afforded $\mathbf{1}$ $(1.594 \mathrm{~g}, 98 \%)$ as a deep blue solid. Mp $232-234^{\circ} \mathrm{C} . R_{\mathrm{f}}=0.33$ (1:1 hexanes/ethyl acetate). UVvis $\left(\mathrm{CH}_{2} \mathrm{Cl}_{2}\right) \lambda_{\max }(\varepsilon): 271$ (22 600), 309 (285 000), 330 (40 200), 440 (4 330), 551 (4 940), 594 (13 400), 645 (25 900) nm. Fluorescence $\left(\mathrm{CH}_{2} \mathrm{Cl}_{2}\right): \lambda_{\max }$ (emission) $=652 \mathrm{~nm}$ using $\lambda$ (excitation) $=309 \mathrm{~nm}$. IR $\left(\mathrm{CDCl}_{3}\right.$, cast) 3500-3100 (s, broad), $3046(\mathrm{w}), 2942(\mathrm{~s}), 2863(\mathrm{~s}), 2135(\mathrm{~m}) \mathrm{cm}^{-1}$. ${ }^{1} \mathrm{H}$ NMR $\left(500 \mathrm{MHz}, \mathrm{CDCl}_{3}\right): \delta 9.35(\mathrm{~s}, 4 \mathrm{H}), 7.99-7.94(\mathrm{~m}, 4 \mathrm{H}), 7.92(\mathrm{~d}, J=8.1 \mathrm{~Hz}, 4 \mathrm{H}), 7.47$ $(\mathrm{d}, J=8.1 \mathrm{~Hz}, 4 \mathrm{H}), 7.43-7.39(\mathrm{~m}, 4 \mathrm{H}), 4.75(\mathrm{~d}, J=4.8 \mathrm{~Hz}, 4 \mathrm{H}), 1.68(\mathrm{t}, J=5.4 \mathrm{~Hz}, 2 \mathrm{H}), 1.57$ (septet, $J=7.4 \mathrm{~Hz}, 4 \mathrm{H}), 1.39(\mathrm{~d}, J=7.3 \mathrm{~Hz}, 12 \mathrm{H}), 1.26(\mathrm{~d}, J=7.3 \mathrm{~Hz}, 12 \mathrm{H}) .{ }^{13} \mathrm{C}$ NMR $(125$ $\left.\mathrm{MHz}, \mathrm{CDCl}_{3}\right): \delta 142.2,135.6,132.6,132.4,130.7,128.7,126.4,126.3,126.2,118.3,105.8$, 105.5, 65.4, 18.2, 18.1, 12.1. ${ }^{13} \mathrm{C}$ NMR (APT, $125 \mathrm{MHz}, \mathrm{CDCl}_{3}$ ): $\delta 142.2(\mathrm{C}), 135.6(\mathrm{CH}), 132.6$ (C), $132.4(\mathrm{C}), 130.7(\mathrm{C}), 128.7(\mathrm{CH}), 126.4(\mathrm{CH}), 126.3(\mathrm{CH}), 126.2(\mathrm{CH}), 118.3(\mathrm{C}), 105.8$ (C), $105.5(\mathrm{C}), 65.4\left(\mathrm{CH}_{2}\right), 18.2\left(\mathrm{CH}_{3}\right), 18.1\left(\mathrm{CH}_{3}\right), 12.1(\mathrm{CH})$. ESI MS $\mathrm{m} / z 789.4\left([\mathrm{M}+\mathrm{Na}]^{+}\right.$, 100). ESI HRMS $m / z$ calcd. for $\mathrm{C}_{52} \mathrm{H}_{54} \mathrm{O}_{2} \mathrm{Si}_{2} \mathrm{Na}\left([\mathrm{M}+\mathrm{Na}]^{+}\right) 789.3555$, found 789.3551. Anal. calcd. for $\mathrm{C}_{52} \mathrm{H}_{54} \mathrm{O}_{2} \mathrm{Si}_{2}: \mathrm{C}, 81.41 ; \mathrm{H}, 7.09$. Found: $\mathrm{C}, 81.26 ; \mathrm{H}, 7.03$. X-Ray structure of $\mathrm{C}_{52} \mathrm{H}_{54} \mathrm{O}_{2} \mathrm{Si}_{2} \cdot 2 \mathrm{CH}_{3} \mathrm{OH}$. Crystals suitable for $\mathrm{X}$-ray were obtain by vapor diffusion of $\mathrm{MeOH}$ into solutions of $\mathbf{6}$ in THF which had been layered by $\mathrm{MeOH}$ and then left standing in the refridgerator at $4{ }^{\circ} \mathrm{C}$ for several days. TGA: $\mathrm{T}_{\mathrm{d}} \approx 365^{\circ} \mathrm{C}$. DSC: $\mathrm{mp}=239.4-241.1^{\circ} \mathrm{C}$; decomposition, $325^{\circ} \mathrm{C}$ (onset) and $375^{\circ} \mathrm{C}$ (peak). 
Synthesis of 2.

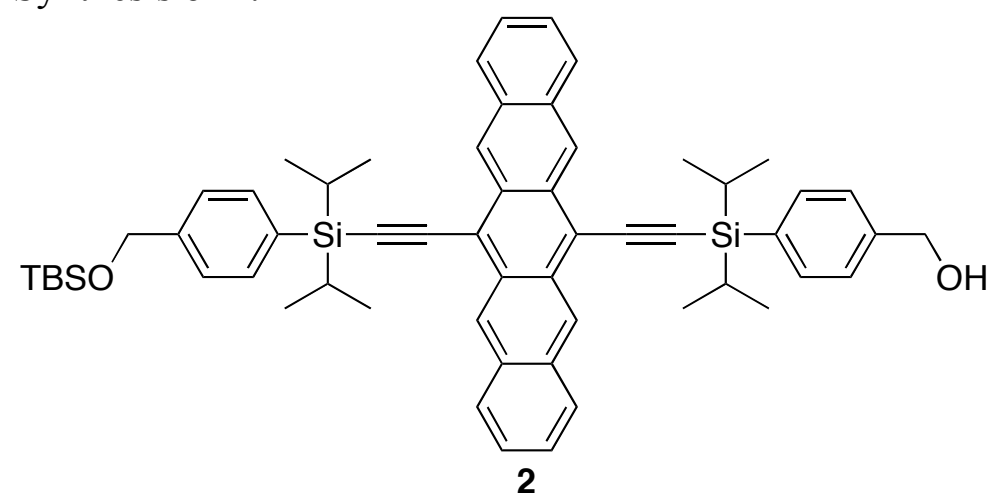

To a solution of $7(1.551 \mathrm{~g}, 1.558 \mathrm{mmol})$ in dry THF $(150 \mathrm{~mL})$ was added dropwise an aqueous solution of $\sim 1.17 \mathrm{M} \mathrm{HCl}$ (4.3 mL; prepared by adding $10.0 \mathrm{~mL}$ of concentrated $\mathrm{HCl}(36 \%)$ to $90.0 \mathrm{~mL}$ of water). The solution was allowed to stir for $45 \mathrm{~min}$ at $\mathrm{rt}$ before being diluted with satd. aq. $\mathrm{NaHCO}_{3}(150 \mathrm{~mL})$ and extracted with $\mathrm{CH}_{2} \mathrm{Cl}_{2}(150 \mathrm{~mL})$. The mixture was separated and the organic phase was washed with $5 \%$ aq. $\mathrm{NaHCO}_{3}(2 \times 150 \mathrm{~mL})$, satd. aq. $\mathrm{NaCl}(200 \mathrm{~mL})$, dried $\left(\mathrm{MgSO}_{4}\right)$, and the solvent removed in vacuo. Column chromatography (silica gel, 5:2 hexanes $\left./ \mathrm{CH}_{2} \mathrm{Cl}_{2}\right)$ afforded unreacted $7(0.552 \mathrm{~g}, 36 \%)$, elution with $\mathrm{CH}_{2} \mathrm{Cl}_{2}$ afforded $2(0.676 \mathrm{~g}$, $49 \%)$ as a deep blue solid, and finally elution with 1:1 ethyl acetate/hexanes afforded $1(0.182 \mathrm{~g}$, $15 \%) . R_{\mathrm{f}}=0.32\left(\mathrm{CH}_{2} \mathrm{Cl}_{2}\right)$. UV-vis $\left(\mathrm{CH}_{2} \mathrm{Cl}_{2}\right) \lambda_{\max }(\varepsilon): 271$ (24 400), 309 (279 000), 329 (40 200), 440 (4 240), 551 (4 860), 593 (13 400), 644 (25 800) nm. IR (CDCl 3 , cast) 3550-3200 (m, broad), 3049 (w), 2952 (s), 2863 (s), 2136 (m) cm ${ }^{-1} .{ }^{1} \mathrm{H}$ NMR (400 MHz, $\mathrm{CDCl}_{3}$ ): $\delta 9.40$ (s, 2H), 9.39 (s, 2H), 8.40-7.97 (m, 4H), $7.94(\mathrm{~d}, J=7.8 \mathrm{~Hz}, 2 \mathrm{H}), 7.92$ (d, $J=7.4 \mathrm{~Hz}, 2 \mathrm{H}), 7.51-7.41$ (m, $8 \mathrm{H}), 4.83(\mathrm{~s}, 2 \mathrm{H}), 4.76(\mathrm{~d}, J=5.93 \mathrm{~Hz}, 2 \mathrm{H}), 1.81(\mathrm{~m}, 1 \mathrm{H}), 1.63(\mathrm{~m}, 4 \mathrm{H}), 1.42(\mathrm{~d}, J=7.3 \mathrm{~Hz}$, $6 \mathrm{H}), 1.42(\mathrm{~d}, J=7.2 \mathrm{~Hz}, 6 \mathrm{H}), 1.29$ (d, $J=7.3 \mathrm{~Hz}, 6 \mathrm{H}), 1.28$ (d, $J=7.3 \mathrm{~Hz}, 6 \mathrm{H}), 0.98$ (s, 9H), $0.14(\mathrm{~s}, 6 \mathrm{H}) .{ }^{13} \mathrm{C}$ NMR $\left(100 \mathrm{MHz}, \mathrm{CDCl}_{3}\right): \delta 142.9,142.2,135.6,135.3,132.6,132.4,132.4$, 131.4, 130.7, 130.7, 128.7, 128.6, 126.4, 126.4, 126.3, 126.2, 126.2, 125.5, 118.4, 118.2, 105.8, $105.8,105.6,105.4,65.4,64.9,26.0,18.4,18.2,18.2,18.2,18.1,12.1,12.1,-5.3 .{ }^{13} \mathrm{C}$ NMR (APT, $100 \mathrm{MHz}, \mathrm{CDCl}_{3}$ ): $\delta 142.9(\mathrm{C}), 142.2(\mathrm{C}), 135.6(\mathrm{CH}), 135.3(\mathrm{CH}), 132.6(\mathrm{C}), 132.4(\mathrm{C})$, $132.4(\mathrm{C}), 131.4(\mathrm{C}), 130.7(\mathrm{C}), 130.7(\mathrm{C}), 128.7(\mathrm{CH}), 128.6(\mathrm{CH}), 126.4(\mathrm{CH}), 126.4(\mathrm{CH})$, $126.3(\mathrm{CH}), 126.2(\mathrm{CH}), 126.2(\mathrm{CH}), 125.5(\mathrm{CH}), 118.4(\mathrm{C}), 118.2(\mathrm{C}), 105.8(\mathrm{C}), 105.8(\mathrm{C})$, 105.6 (C), 105.4 (C), $65.4\left(\mathrm{CH}_{2}\right), 64.9\left(\mathrm{CH}_{2}\right), 26.0\left(\mathrm{CH}_{3}\right), 18.4(\mathrm{C}), 18.2\left(\mathrm{CH}_{3}\right), 18.2\left(\mathrm{CH}_{3}\right), 18.2$ $\left(\mathrm{CH}_{3}\right), 18.1\left(\mathrm{CH}_{3}\right), 12.1(\mathrm{CH}), 12.1(\mathrm{CH}),-5.3\left(\mathrm{CH}_{3}\right)$. ESI MS m/z $881.5\left([\mathrm{M}+\mathrm{H}]^{+}, 100\right), 880.5$ ([M $]^{+}$, 20). ESI HRMS $m / z$ calcd. for $\mathrm{C}_{58} \mathrm{H}_{69} \mathrm{O}_{2} \mathrm{Si}_{3}\left([\mathrm{M}+\mathrm{H}]^{+}\right) 881.4600$, found 881.4607. TGA (heating rate of $20^{\circ} \mathrm{C} / \mathrm{min}$ ): $\mathrm{T}_{\mathrm{d}} \approx 385^{\circ} \mathrm{C}$. DSC: decomposition, $360{ }^{\circ} \mathrm{C}$ (onset) and $380{ }^{\circ} \mathrm{C}$ (peak).

Synthesis of 4-bromobenzyl alcohol (S2).

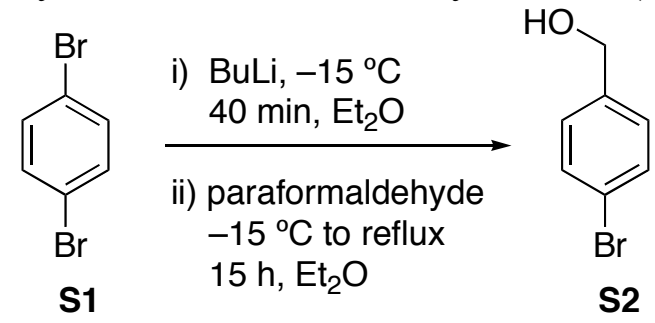

To a solution of 1,4-dibromobenzene (S1) $(58.98 \mathrm{~g}, 0.2500 \mathrm{~mol})$ in dry ether $(500 \mathrm{~mL})$ cooled to $-15^{\circ} \mathrm{C}$ was added slowly BuLi (2.5 M in hexanes, $\left.100 \mathrm{~mL}, 0.25 \mathrm{~mol}\right)$ over a period of $40 \mathrm{~min}$. 
To this solution was added paraformaldehyde $(8.26 \mathrm{~g}, 0.275 \mathrm{~mol})$ which been dried in a vacuum desiccator over $\mathrm{P}_{2} \mathrm{O}_{5}{ }^{2}$. This solution was cautiously allowed to warm up to rt over a period of $1 \mathrm{~h}$ (Cautionary Note: for large scale reactions ( $>5 \mathrm{~g}$ of S1), an acetone/dry-ice cooling bath was kept on hand, and used as necessary to keep the exothermic reaction from getting out of control) before refluxing the solution for $15 \mathrm{~h}$. The reaction was then poured into ice-water and extracted with $\mathrm{CH}_{2} \mathrm{Cl}_{2}$. The organic phase was washed with satd. aq. $\mathrm{NaCl}$, dried $\left(\mathrm{MgSO}_{4}\right)$, and the solvent removed in vacuo. The crude solid was purified by recrystallization from $\mathrm{CHCl}_{2}$ and hexanes to afford 4-bromobenzyl alcohol (S2) $(30.25 \mathrm{~g}, 65 \%)$ as a white crystalline solid. The ${ }^{1} \mathrm{H}$ - and ${ }^{13} \mathrm{C}$ NMR spectra were in agreement with commercially available samples.

Synthesis of 4-bromobenzyl tert-butyldimethylsilyl ether (3).

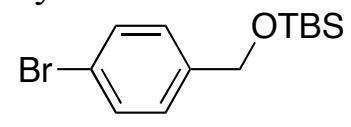

3

This procedure was modified from that reported by Sessler, J. L.; Wang, B.; Harriman, A. J. Am. Chem. Soc. 1995, 117, 707-714 by changing the solvent from anhydrous DMF to anhydrous $\mathrm{CH}_{3} \mathrm{CN}$. Additionally the crude product was purified via vacuum distillation (bp $135-140{ }^{\circ} \mathrm{C}, \sim 1$ $\mathrm{mmHg}$ ) instead of column chromatography to afford a clear colorless liquid in $90 \%$ yield. The ${ }^{1} \mathrm{H}$ - and ${ }^{13} \mathrm{C}$-NMR spectra were in agreement with literature data.

Synthesis of 4.

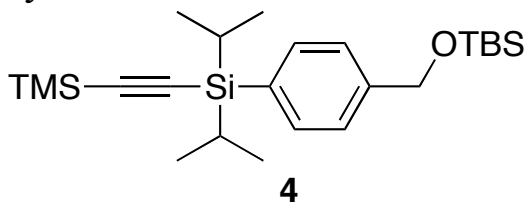

To a solution of $3(17.64 \mathrm{~g}, 58.55 \mathrm{mmol})$ in dry THF $(200 \mathrm{~mL})$ under $\mathrm{N}_{2}$ atmosphere at $-78{ }^{\circ} \mathrm{C}$ was added dropwise $\mathrm{BuLi}(2.5 \mathrm{M}$ in hexanes, $23.4 \mathrm{~mL}, 58.5 \mathrm{mmol})$. The solution was stirred for 30 min before being transferred via cannula to a solution of dichlorodiisopropylsilane ( $11.0 \mathrm{~g}$, $10.7 \mathrm{~mL}, 59.3 \mathrm{mmol})$ in dry THF $(70 \mathrm{~mL})$ at $-78^{\circ} \mathrm{C}$. The mixture was warmed to $\mathrm{rt}$ and stirred for $5.5 \mathrm{~h}$. In a second flask, to a solution of (trimethylsilyl)acetylene $(14.5 \mathrm{~mL}, 10.1 \mathrm{~g}, 102$ mmol) in dry THF $(150 \mathrm{~mL})$ under $\mathrm{N}_{2}$ atmosphere at $-78^{\circ} \mathrm{C}$ was added dropwise $\mathrm{BuLi}(2.5 \mathrm{M}$ in hexanes, $38.6 \mathrm{~mL}, 96.5 \mathrm{mmol}$ ). This solution was warmed to $0{ }^{\circ} \mathrm{C}$ and transferred via cannula into the first mixture which had been cooled to $-78^{\circ} \mathrm{C}$. The combined reaction was then allowed to warm to rt and stir for $16 \mathrm{~h}$. The reaction was cooled to $-15^{\circ} \mathrm{C}$ before quenching with satd. aq. $\mathrm{NH}_{4} \mathrm{Cl}$. The mixture was extracted with hexanes (2x), and the organic phase was washed with satd. aq. $\mathrm{NaCl}(2 \mathrm{x})$, dried $\left(\mathrm{MgSO}_{4}\right)$, and the solvent removed in vacuo. The crude oil was purified by vacuum distillation by collecting the distillates with bp $125-170{ }^{\circ} \mathrm{C}(\sim 1 \mathrm{mmHg})$ and afforded a mixture of $\mathbf{4}$ and $\mathbf{5}$ (65:35 by ${ }^{1} \mathrm{H}$ NMR). A mixture of $\mathbf{4}$ and $\mathbf{5}$ can be subjected to desilylation as described for $\mathbf{4}$ (see below). Note: Column chromatography (silica gel, 4:1 hexanes $\left./ \mathrm{CH}_{2} \mathrm{Cl}_{2}\right)$ can be used to obtain 4 pure as a clear colorless liquid. $\mathrm{Bp} 155-165{ }^{\circ} \mathrm{C}(\sim 1$ mmHg). $R_{\mathrm{f}}=0.71\left(7: 3\right.$ hexanes $\left./ \mathrm{CH}_{2} \mathrm{Cl}_{2}\right)$. IR $\left(\mathrm{CDCl}_{3}\right.$, cast): $3071(\mathrm{w}), 3015(\mathrm{w}), 2956(\mathrm{~s}), 2894$ (s), 2864 (s), $1251(\mathrm{~s}) \mathrm{cm}^{-1} .{ }^{1} \mathrm{H}$ NMR $\left(400 \mathrm{MHz}, \mathrm{CDCl}_{3}\right): \delta 7.56(\mathrm{~d}, J=7.6 \mathrm{~Hz}, 2 \mathrm{H}), 7.30(\mathrm{~d}, J=$ $7.6 \mathrm{~Hz}, 2 \mathrm{H}$ ), 4.74 (s, 1H), 1.19 (septet, $J=7.2 \mathrm{~Hz}, 2 \mathrm{H}$ ), 1.05 (d, $J=7.2 \mathrm{~Hz}, 6 \mathrm{H}$ ), 0.95 (d, $J=7.2$

${ }^{2}$ Amarego, W. L. F.; Chai, C. L. L. Purification of Laboratory Chemicals, $5^{\text {th }}$ ed.; Elsevier: New York, 2003; pp 245. 
$\mathrm{Hz}, 6 \mathrm{H}), 0.94$ (s, 9H), $0.21(\mathrm{~s}, 9 \mathrm{H}), 0.09$ (s, 6H). ${ }^{13} \mathrm{C} \mathrm{NMR}\left(100 \mathrm{MHz}, \mathrm{CDCl}_{3}\right): \delta 142.5,135.0$, 131.4, 125.2, 117.6, 108.4, 64.9, 26.0, 18.4, 17.8, 17.7, 11.5, 0.0, -5.3. ${ }^{13} \mathrm{C}$ NMR (APT, 100 $\left.\mathrm{MHz}, \mathrm{CDCl}_{3}\right): \delta 142.5(\mathrm{C}), 135.0(\mathrm{CH}), 131.4(\mathrm{C}), 125.2(\mathrm{CH}), 117.6(\mathrm{C}), 108.4(\mathrm{C}), 64.9\left(\mathrm{CH}_{2}\right)$, $26.0\left(\mathrm{CH}_{3}\right), 18.4(\mathrm{C}), 17.7\left(\mathrm{CH}_{3}\right), 17.7\left(\mathrm{CH}_{3}\right), 11.5(\mathrm{CH}), 0.0\left(\mathrm{CH}_{3}\right),-5.3\left(\mathrm{CH}_{3}\right)$. EIMS $m / z 431.2$ $\left([\mathrm{M}-\mathrm{H}]^{+}, 2\right), 389.2\left([\mathrm{M}-i-\mathrm{Pr}]^{+}, 73\right), 375.2\left([\mathrm{M}-t-\mathrm{Bu}]^{+}, 100\right)$. EI HRMS calcd. for $\mathrm{C}_{21} \mathrm{H}_{37} \mathrm{OSi}_{3}$ $\left([\mathrm{M}-i-\mathrm{Pr}]^{+}\right)$389.2152, found 389.2148. Anal. calcd. for $\mathrm{C}_{24} \mathrm{H}_{44} \mathrm{OSi}_{3}: \mathrm{C}, 66.59 ; \mathrm{H}, 10.25$. Found: C, 66.81; H, 10.39.

Synthesis of 5.

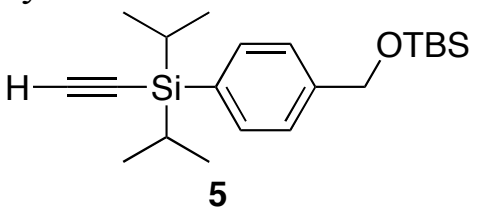

To a solution of $4(3.660 \mathrm{~g}, 8.456 \mathrm{~mol})$ in wet $\mathrm{MeOH}(160 \mathrm{~mL})$ was added $\mathrm{K}_{2} \mathrm{CO}_{3}(1.264 \mathrm{~g}$, $9.146 \mathrm{mmol})$ and water $(2 \mathrm{~mL})$. The mixture was stirred for $1 \mathrm{~h} \mathrm{at} \mathrm{rt}$, before adding water (100 $\mathrm{mL})$ and extracting the mixture with hexanes $(2 \mathrm{x})$. The organic phase was washed with satd. aq. $\mathrm{NaCl}(2 \mathrm{x})$, dried $\left(\mathrm{MgSO}_{4}\right)$, and the solvent removed in vacuo. No purification was required to obtain $5(2.847 \mathrm{~g}, 93 \%)$ as a clear colorless liquid. Bp $145-155^{\circ} \mathrm{C}(\sim 1 \mathrm{mmHg}) . R_{\mathrm{f}}=0.62(7: 3$ hexanes $/ \mathrm{CH}_{2} \mathrm{Cl}_{2}$ ). IR $\left(\mathrm{CH}_{2} \mathrm{Cl}_{2}\right.$, cast): 3293 (m), 3072 (w), 3016 (w), 2956 (s), 2930 (s), 2865 (s), $2035(\mathrm{~m}) \mathrm{cm}^{-1} .{ }^{1} \mathrm{H}$ NMR $\left(500 \mathrm{MHz}, \mathrm{CDCl}_{3}\right): \delta 7.58(\mathrm{~d}, J=8.0 \mathrm{~Hz}, 2 \mathrm{H}), 7.32(\mathrm{~d}, J=7.7 \mathrm{~Hz}, 2 \mathrm{H})$, $4.75(\mathrm{~s}, 1 \mathrm{H}), 2.50(\mathrm{~s}, 1 \mathrm{H}), 1.23$ (septet, $J=7.3 \mathrm{~Hz}, 2 \mathrm{H}), 1.07$ (d, $J=7.2,6 \mathrm{H}), 0.98$ (d, $J=7.3$, 6H), 0.94 (s, 9H), 0.09 (s, 6H). ${ }^{13} \mathrm{C}$ NMR (125 MHz, $\left.\mathrm{CDCl}_{3}\right): \delta$ 142.7, 135.0, 130.8, 125.3, 96.0, 84.9, 64.9, 26.0, 18.4, 17.7, 17.6, 11.5, -5.3. NMR (APT, $125 \mathrm{MHz}, \mathrm{CDCl}_{3}$ ): $\delta 142.7$ (C), 135.0 $(\mathrm{CH}), 130.8(\mathrm{C}), 125.3(\mathrm{CH}), 96.0(\mathrm{CH}),{ }^{3} 84.9(\mathrm{C}), 64.9\left(\mathrm{CH}_{2}\right), 26.0\left(\mathrm{CH}_{3}\right), 18.4(\mathrm{C}), 17.7\left(\mathrm{CH}_{3}\right)$, $17.6\left(\mathrm{CH}_{3}\right), 11.5(\mathrm{CH}),-5.3\left(\mathrm{CH}_{3}\right)$. EIMS $m / z 360.2\left(\mathrm{M}^{+}, 1\right), 303.2\left(\left[\mathrm{M}^{+}-t-\mathrm{Bu}\right]^{+}, 100\right)$. EI HRMS calcd. for $\mathrm{C}_{21} \mathrm{H}_{36} \mathrm{OSi}_{2}\left(\mathrm{M}^{+}\right)$360.2305, found 360.2298. Anal. calcd. for $\mathrm{C}_{21} \mathrm{H}_{36} \mathrm{OSi}_{2}$ : C, 69.93; H, 10.06. Found: C, 70.00; H, 10.04.

${ }^{3}$ This carbon signal appears on the wrong side of the APT spectrum (i.e., it is observed on the $\mathrm{C} / \mathrm{CH}_{2}$ side). It is, however, the terminal alkyne carbon, which has been confirmed by gHMQC and $\mathrm{gHMBC}$ experiments. 
Synthesis of trans- and cis-6.

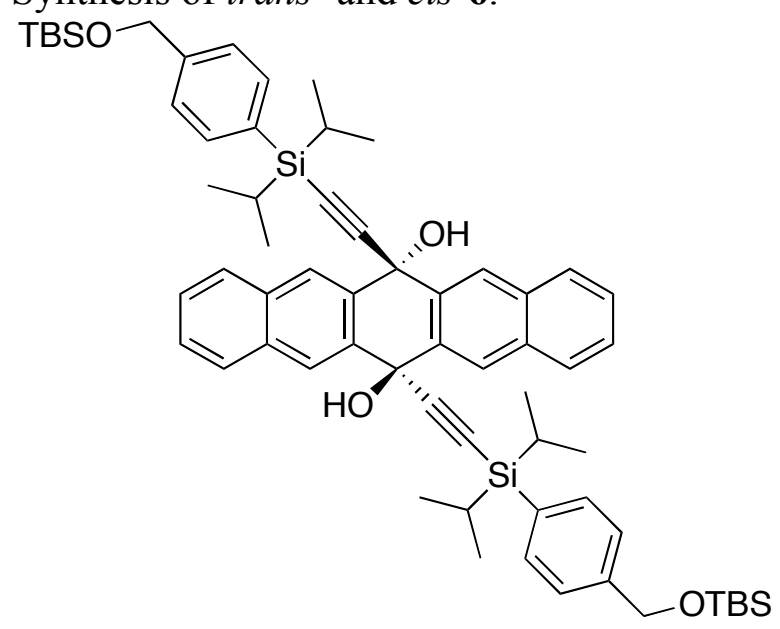

trans-6

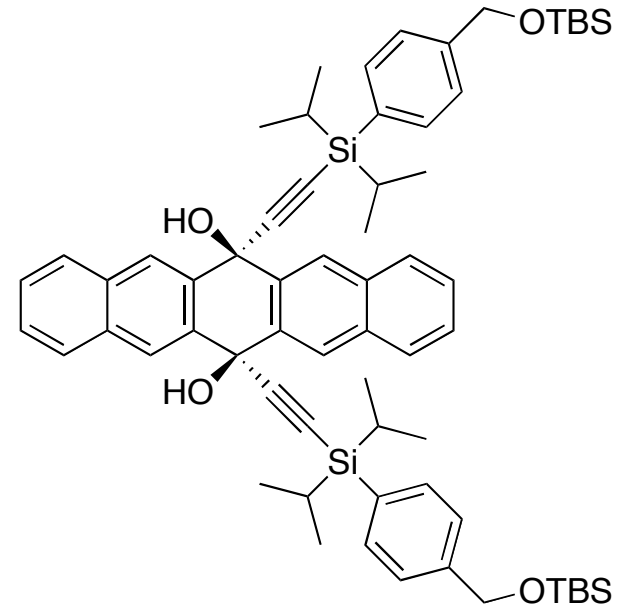

cis-6

To a solution of $5(12.70 \mathrm{~g}, 35.22 \mathrm{mmol})$ in THF $(40 \mathrm{~mL})$ cooled to $-78{ }^{\circ} \mathrm{C}$ was added dropwise BuLi (2.5 M in hexanes, $13.6 \mathrm{~mL}, 34.0 \mathrm{mmol})$. The solution was allowed to stir for $30 \mathrm{~min}$ before being transferred via cannula into a suspension of 6,13-pentacenequinone (1.780 g, 5.772 $\mathrm{mmol})$ in THF $(20 \mathrm{~mL})$ at $-15^{\circ} \mathrm{C}$. The reaction mixture was then allowed to warm up to $\mathrm{rt}$ and stir for an additional $3 \mathrm{~h}$. The reaction was quenched at $-78{ }^{\circ} \mathrm{C}$ with satd. aq. $\mathrm{NH}_{4} \mathrm{Cl}(15 \mathrm{~mL})$ and allowed to warm up to $\mathrm{rt}$. Upon reaching $\mathrm{rt}$, water $(100 \mathrm{~mL})$ was added followed by $\mathrm{CH}_{2} \mathrm{Cl}_{2}(150$ $\mathrm{mL})$. The mixture was separated and the organic phase was washed with satd. aq. $\mathrm{NaCl}(75 \mathrm{~mL})$, dried $\left(\mathrm{MgSO}_{4}\right)$, and the solvent removed in vacuo. Column chromatography (silica gel, 7:3 hexanes $/ \mathrm{CH}_{2} \mathrm{Cl}_{2}$ ) was used to recover excess $\mathbf{5}$, the column was then eluted with 2:1 $\mathrm{CH}_{2} \mathrm{Cl}_{2}$ /hexanes to afford trans-6 (5.206 g, 88\%) as a white foamy solid (light sensitive; 2 spots will form on a TLC if the spot is irradiated even for one second with a $254 \mathrm{~nm}$ TLC UV lamp before eluting the TLC, the second spot appears at $\left.\mathrm{R}_{\mathrm{f}}=0.10\left(\mathrm{CH}_{2} \mathrm{Cl}_{2}\right)\right)$. The column was then eluted with $\mathrm{CH}_{2} \mathrm{Cl}_{2}$ to afford crude cis-6 which upon following two additional column chromatographic separations (silica gel, $\mathrm{CH}_{2} \mathrm{Cl}_{2}$ and silica, 4:1 hexanes/ethyl acetate) gave cis-6 $(0.22 \mathrm{~g}, 4 \%)$ as a pale yellow oil.

Trans-6. $R_{\mathrm{f}}=0.80\left(\mathrm{CH}_{2} \mathrm{Cl}_{2}\right)$. IR $\left(\mathrm{CDCl}_{3}\right.$, cast $) 3600-3300(\mathrm{~m}$, broad $), 3056(\mathrm{w}), 3018(\mathrm{w}), 2951$ (s), 2891 (s), 2862 (s), 2171 (w), $1254(\mathrm{~m}) \mathrm{cm}^{-1}$. ${ }^{1} \mathrm{H}$ NMR (400 MHz, $\mathrm{CDCl}_{3}$ ): $\delta 8.80$ (s, 4H), $7.98-7.92(\mathrm{~m}, 4 \mathrm{H}), 7.62(\mathrm{~d}, J=8.0 \mathrm{~Hz}, 4 \mathrm{H}), 7.58-7.54(\mathrm{~m}, 4 \mathrm{H}), 7.31(\mathrm{~d}, J=8.1 \mathrm{~Hz}, 4 \mathrm{H}), 4.76$ (s, 4H), 3.48 (s, 2H), 1.32 (septet, $J=7.3 \mathrm{~Hz}, 4 \mathrm{H}), 1.14$ (d, $J=7.3 \mathrm{~Hz}, 12 \mathrm{H}), 1.05$ (d, $J=7.3 \mathrm{~Hz}$, $12 \mathrm{H}), 0.99(\mathrm{~s}, 18 \mathrm{H}), 0.14(\mathrm{~s}, 12 \mathrm{H}) .{ }^{13} \mathrm{C} \mathrm{NMR}\left(100 \mathrm{MHz}, \mathrm{CDCl}_{3}\right): \delta 142.7,136.2,135.0,133.3$, 130.9, 128.2, 126.9, 126.2, 125.3, 110.6, 88.1, 69.8, 64.8, 26.0, 18.4, 17.9, 17.8, 11.7, -5.3. (APT, $\left.100 \mathrm{MHz}, \mathrm{CDCl}_{3}\right)$ : $\delta 142.7(\mathrm{C}), 136.2(\mathrm{C}), 135.0(\mathrm{CH}), 133.2(\mathrm{C}), 130.8(\mathrm{C}), 128.2(\mathrm{CH})$, $126.9(\mathrm{CH}), 126.2(\mathrm{CH}), 125.3(\mathrm{CH}), 110.6(\mathrm{C}), 88.1(\mathrm{C}), 69.8(\mathrm{C}), 64.8\left(\mathrm{CH}_{2}\right), 26.0\left(\mathrm{CH}_{3}\right), 18.4$ (C), $17.9\left(\mathrm{CH}_{3}\right), 17.8\left(\mathrm{CH}_{3}\right), 11.7(\mathrm{CH}),-5.3\left(\mathrm{CH}_{3}\right)$. ESI MS $m / z 1051.5\left([\mathrm{M}+\mathrm{Na}]^{+}, 100\right)$, $1011.6\left([\mathrm{M}-\mathrm{OH}]^{+}, 23\right)$. ESI HRMS $m / z$ calcd. for $\mathrm{C}_{64} \mathrm{H}_{84} \mathrm{O}_{4} \mathrm{Si}_{4} \mathrm{Na}\left([\mathrm{M}+\mathrm{Na}]^{+}\right) 1051.5339$, found 1051.5337. Anal. calcd. for $\mathrm{C}_{64} \mathrm{H}_{84} \mathrm{O}_{4} \mathrm{Si}_{4}$ : C, 74.65; H, 8.22. Found: C, 74.26; H, 8.50.

\footnotetext{
${ }^{4}$ Tentative assignment of relative geometry of alcohol moieties is made on the basis of relative polarity of the two products on silica gel and the fact that trans-6 is centrosymmetric, see footnote 8 in the paper for details.
} 
Cis-6. $R_{\mathrm{f}}=0.35\left(\mathrm{CH}_{2} \mathrm{Cl}_{2}\right), 0.60$ (4:1 hexanes/ethyl acetate). IR $\left(\mathrm{CDCl}_{3}\right.$, cast $) 3504(\mathrm{w}), 3450-$ 3100 (w, broad), 3057 (w), 3018 (w), 2953 (s), 2863 (s), 2169 (w), $1256(\mathrm{~m}) \mathrm{cm}^{-1}$. ${ }^{1} \mathrm{H}$ NMR (500 $\left.\mathrm{MHz}, \mathrm{CDCl}_{3}\right): \delta 8.84(\mathrm{~s}, 4 \mathrm{H}), 7.88-7.83(\mathrm{~m}, 4 \mathrm{H}), 7.80(\mathrm{~d}, J=8.0 \mathrm{~Hz}, 4 \mathrm{H}), 7.56-7.51(\mathrm{~m}, 4 \mathrm{H})$, 7.39 (d, $J=7.5 \mathrm{~Hz}, 4 \mathrm{H}), 4.79$ (s, 4H), 4.47 (s, 2H), 1.48 (septet, $J=7.3 \mathrm{~Hz}, 4 \mathrm{H}), 1.32$ (d, $J=7.3$ $\mathrm{Hz}, 12 \mathrm{H}), 1.19$ (d, $J=7.3 \mathrm{~Hz}, 12 \mathrm{H}), 0.95(\mathrm{~s}, 18 \mathrm{H}), 0.11(\mathrm{~s}, 12 \mathrm{H}) .{ }^{13} \mathrm{C} \mathrm{NMR}\left(125 \mathrm{MHz}, \mathrm{CDCl}_{3}\right)$ : $\delta 143.0,135.7,135.2,133.1,130.9,128.3,127.5,127.2,125.5,106.8,91.0,72.4,64.9,26.0$, 18.4, 18.1, 18.0, 11.9, -5.3. NMR (APT, $\left.125 \mathrm{MHz}, \mathrm{CDCl}_{3}\right)$ : $\delta 143.0(\mathrm{C}), 135.7$ (C), $135.2(\mathrm{CH})$, $133.1(\mathrm{C}), 130.9(\mathrm{C}), 128.3(\mathrm{CH}), 127.5(\mathrm{CH}), 127.1(\mathrm{CH}), 125.4(\mathrm{CH}), 106.8(\mathrm{C}), 91.0(\mathrm{C}), 72.4$ (C), $64.9\left(\mathrm{CH}_{2}\right), 26.0\left(\mathrm{CH}_{3}\right), 18.4(\mathrm{C}), 18.1\left(\mathrm{CH}_{3}\right), 18.0\left(\mathrm{CH}_{3}\right), 11.9(\mathrm{CH}),-5.3\left(\mathrm{CH}_{3}\right)$. ESI MS $m / z 1051.6\left([\mathrm{M}+\mathrm{Na}]^{+}, 100\right), 1011.6\left([\mathrm{M}-\mathrm{OH}]^{+}, 23\right)$. ESI MS $m / z 1051.5\left([\mathrm{M}+\mathrm{Na}]^{+}, 30\right)$, $1011.6\left([\mathrm{M}-\mathrm{OH}]^{+}, 100\right)$. ESI HRMS $m / z$ calcd. for $\mathrm{C}_{64} \mathrm{H}_{84} \mathrm{O}_{4} \mathrm{Si}_{4} \mathrm{Na}\left([\mathrm{M}+\mathrm{Na}]^{+}\right) 1051.5339$, found 1051.5335 .

Synthesis of 7.

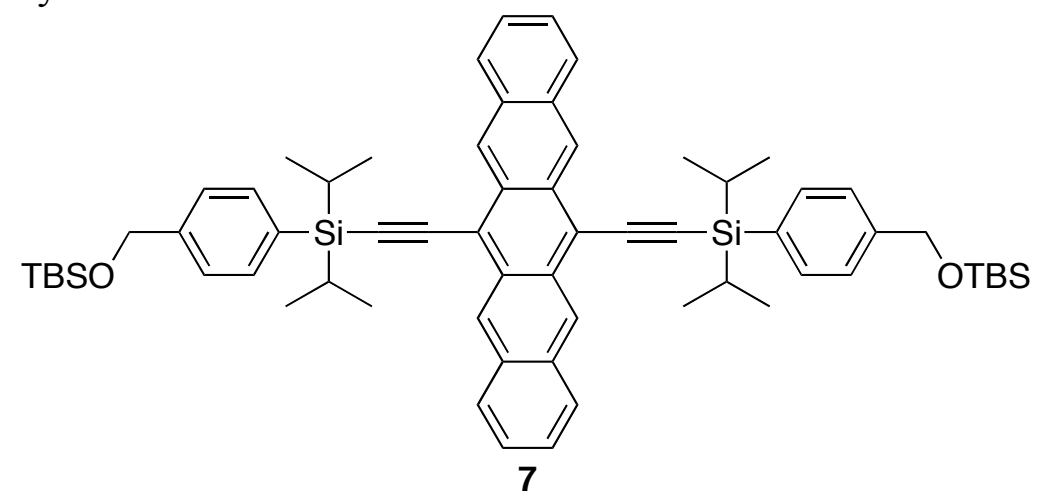

To a solution of trans $-6(3.10 \mathrm{~g}, 3.01 \mathrm{mmol})$ in dry THF $(150 \mathrm{~mL})$ that had been deoxygenated by bubbling $\mathrm{N}_{2}$ for 15 minutes was added dropwise a solution of $\mathrm{SnCl}_{2} \cdot 2 \mathrm{H}_{2} \mathrm{O}(1.78 \mathrm{~g}, 7.89$ mmol) in THF (10 mL). After addition of the $\mathrm{Sn}_{2} \mathrm{Cl}_{2}$, the solution was continued to be deoxygenated for another $10 \mathrm{~min}$ and allowed to stir at $\mathrm{rt}$ for a total of $6.5 \mathrm{~h}$ before adding water $(125 \mathrm{~mL})$ and then $\mathrm{CH}_{2} \mathrm{Cl}_{2}(200 \mathrm{~mL})$. The mixture was separated and the organic phase was washed with satd. aq. $\mathrm{NaCl}(70 \mathrm{~mL})$, dried $\left(\mathrm{MgSO}_{4}\right)$, and the solvent removed in vacuo. Column chromatography (silica gel, 5:2 hexanes/ $\left.\mathrm{CH}_{2} \mathrm{Cl}_{2}\right)$ afforded $7(2.50 \mathrm{~g}, 83 \%)$ as a deep blue solid. Mp $180-182^{\circ} \mathrm{C} . R_{\mathrm{f}}=0.45$ (7:3 hexanes/CH $\left.\mathrm{CH}_{2} \mathrm{Cl}_{2}\right)$. UV-vis $\left(\mathrm{CH}_{2} \mathrm{Cl}_{2}\right) \lambda_{\max }(\varepsilon): 271$ (21 500), 309 (303 000), 330 (41 300), 440 (4 430), 551 (5 000), 594 (13 900), 645 (27 100) nm. IR ( $\mathrm{CDCl}_{3}$, cast) $3050(\mathrm{w}), 2953$ (s), 2890 (s), 2862 (s), $2137(\mathrm{~m}), 1257(\mathrm{~m}) \mathrm{cm}^{-1} .{ }^{1} \mathrm{H}$ NMR (400 MHz, $\left.\mathrm{CDCl}_{3}\right): \delta 9.36(\mathrm{~s}, 4 \mathrm{H}), 8.00-7.94(\mathrm{~m}, 4 \mathrm{H}), 7.89(\mathrm{~d}, J=8.0 \mathrm{~Hz}, 4 \mathrm{H}), 7.46-7.38(\mathrm{~m}, 8 \mathrm{H}), 4.81(\mathrm{~s}$, 4H), 1.56 (septet, $J=7.2 \mathrm{~Hz}, 4 \mathrm{H}), 1.39$ (d, $J=7.3 \mathrm{~Hz}, 12 \mathrm{H}), 1.26$ (d, $J=7.3 \mathrm{~Hz}, 12 \mathrm{H}), 0.95$ (s, 18H), $0.12(\mathrm{~s}, 12 \mathrm{H}) .{ }^{13} \mathrm{C}$ NMR $\left(100 \mathrm{MHz}, \mathrm{CDCl}_{3}\right): \delta 142.9,135.3,132.4,131.5,130.7,128.7$, 126.3, 126.2, 125.5, 118.3, 105.7, 105.7, 64.9, 26.0, 18.5, 18.3, 18.2, 12.1, -5.2. ${ }^{13} \mathrm{C}$ NMR (APT, $\left.100 \mathrm{MHz}, \mathrm{CDCl}_{3}\right): \delta 142.9(\mathrm{C}), 135.3(\mathrm{CH}), 132.4(\mathrm{C}), 131.4(\mathrm{C}), 130.7(\mathrm{C}), 128.7(\mathrm{CH}), 126.3$ $(\mathrm{CH}), 126.2(\mathrm{CH}), 125.5(\mathrm{CH}), 118.3(\mathrm{C}), 105.7(\mathrm{C}) 64.9\left(\mathrm{CH}_{2}\right), 26.0\left(\mathrm{CH}_{3}\right), 18.3\left(\mathrm{CH}_{3}\right), 18.2$ $\left(\mathrm{CH}_{3}\right), 12.1(\mathrm{CH}),-5.2\left(\mathrm{CH}_{3}\right)$ (two signals not observed). ESI MS $m / z 1017.6\left([\mathrm{M}+\mathrm{Na}]^{+}, 45\right)$, 995.5 $\left([\mathrm{M}+\mathrm{H}]^{+}, 100\right), 994.5\left(\mathrm{M}^{+}, 50\right)$. ESI HRMS $m / z$ calcd. for $\mathrm{C}_{64} \mathrm{H}_{83} \mathrm{O}_{2} \mathrm{Si}_{4}\left([\mathrm{M}+\mathrm{H}]^{+}\right)$ 995.5465, found 995.5462. Anal. calcd. for $\mathrm{C}_{64} \mathrm{H}_{82} \mathrm{O}_{2} \mathrm{Si}_{4}: \mathrm{C}, 77.20 ; \mathrm{H}, 8.30$. Found: C, 77.21; $\mathrm{H}$, 8.33. TGA: $\mathrm{T}_{\mathrm{d}} \approx 385^{\circ} \mathrm{C}$. DSC: $\mathrm{mp}=180.3-183.0{ }^{\circ} \mathrm{C}$. 
Synthesis of polymer $\mathbf{8}$.

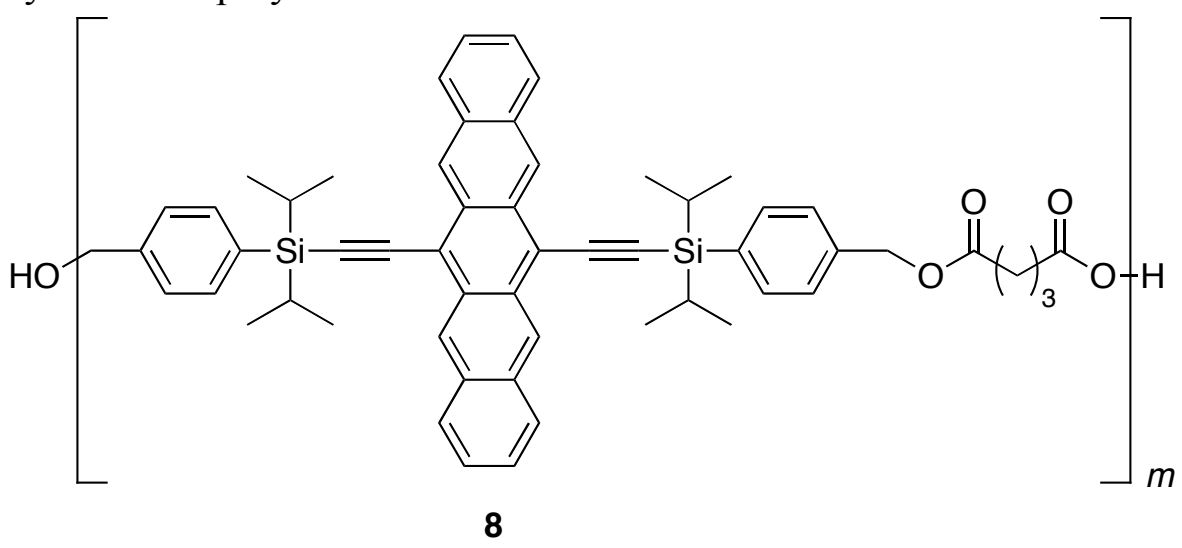

To a solution of $1\left(0.105 \mathrm{~g}, 0.137 \mathrm{mmol}\right.$, freshly recrystallized from $\mathrm{CH}_{2} \mathrm{Cl}_{2} /$ hexanes $)$ and DMAP $(0.080 \mathrm{~g}, 0.65 \mathrm{mmol})$ in dry $\mathrm{CH}_{2} \mathrm{Cl}_{2}(2.5 \mathrm{~mL})$ was added quickly $0.28 \mathrm{~mL}(0.077 \mathrm{mmol})$ of a solution $0.27 \mathrm{M}$ glutaryl chloride [glutaryl chloride $(0.46 \mathrm{~g}, 0.35 \mathrm{~mL}, 2.7 \mathrm{mmol})$ dissolved in dry $\mathrm{CH}_{2} \mathrm{Cl}_{2}(10 \mathrm{~mL})$ under $\mathrm{N}_{2}$ atmosphere] and after $\sim 30$ seconds of stirring at $\mathrm{rt}$ a second addition of $0.25 \mathrm{~mL}(0.069 \mathrm{mmol})$ of the $0.27 \mathrm{M}$ glutaryl chloride solution was added quickly to the reaction mixture. The reaction was then allowed to stir for $3 \mathrm{~h}$ before being diluted with $\mathrm{CH}_{2} \mathrm{Cl}_{2}(50 \mathrm{~mL})$ and satd. aq. $\mathrm{NaHCO}_{3}(100 \mathrm{~mL})$. The mixture was separated and the organic phase was washed with $5 \%$ aq. $\mathrm{NaHCO}_{3}(1 \times 100 \mathrm{~mL})$, satd. aq. $\mathrm{NH}_{4} \mathrm{Cl}(2 \mathrm{x} 150 \mathrm{~mL})$, satd. aq. $\mathrm{NaCl}(150 \mathrm{~mL})$, dried $\left(\mathrm{MgSO}_{4}\right)$, and the solvent removed in vacuo. The residue was dissolved in $\mathrm{CH}_{2} \mathrm{Cl}_{2}(2 \mathrm{~mL})$, and precipitated by adding hexanes $(40 \mathrm{~mL})$ and cooling to $-78{ }^{\circ} \mathrm{C}$. After filtering, the resulting solid was washed with hexanes $(3 \times 15 \mathrm{~mL})$ and redissolved in $\mathrm{CH}_{2} \mathrm{Cl}_{2}$. This solution was filtered through a fritted funnel to remove any undissolved solids, concentrated in vacuo, and the resulting solid dried under vacuum to afford polymer $\mathbf{8}(0.087 \mathrm{~g}, 72 \%)$ as a deep blue solid. UV-vis $\left(\mathrm{CH}_{2} \mathrm{Cl}_{2}\right) \lambda_{\max }: 271,309,330,440,552,594,645 \mathrm{~nm}$. IR $\left(\mathrm{CDCl}_{3}\right.$, cast $)$ 3042 (w), 3020 (w), 2943 (s), 2864 (s), 2136 (m), 1738 (s) $\mathrm{cm}^{-1}$. UV-vis $\left(\mathrm{CH}_{2} \mathrm{Cl}_{2}\right) \lambda_{\max }$ : 271, 309, 330, 440, 552, 594, 645 nm. ${ }^{1} \mathrm{H}$ NMR (500 MHz, $\left.\mathrm{CDCl}_{3}\right)$ : $\delta 9.35$ (bs, 4H), 7.99-7.94 (m, 4H), 7.88 (broad d, $J=7.9 \mathrm{~Hz}, 4 \mathrm{H}), 7.46-7.38(\mathrm{~m}, 8 \mathrm{H}), 5.17(\mathrm{bs}, 4 \mathrm{H}), 2.47(\mathrm{t}, J=7.4 \mathrm{~Hz}, 4 \mathrm{H})$, 2.04 (quintet, $J=7.3 \mathrm{~Hz}, 2 \mathrm{H}), 1.61-1.48(\mathrm{~m}, 4 \mathrm{H}), 1.38$ (broad d, $J=7.3 \mathrm{~Hz}, 12 \mathrm{H}), 1.25(\mathrm{~d}, J=$ $7.4 \mathrm{~Hz}, 12 \mathrm{H}) .{ }^{13} \mathrm{C} \mathrm{NMR}\left(125 \mathrm{MHz}, \mathrm{CDCl}_{3}\right): \delta 172.7,137.1,135.6,133.4,132.4,130.7,128.6$, $127.5,126.2,118.2,105.9,105.3,66.2,33.3,20.1,18.2,18.1,12.0$. TGA: $_{\mathrm{d}} \approx 385^{\circ} \mathrm{C}$. DSC: decomposition, $370{ }^{\circ} \mathrm{C}$ (onset) and $405^{\circ} \mathrm{C}$ (peak). 
Synthesis of polymer 9 .

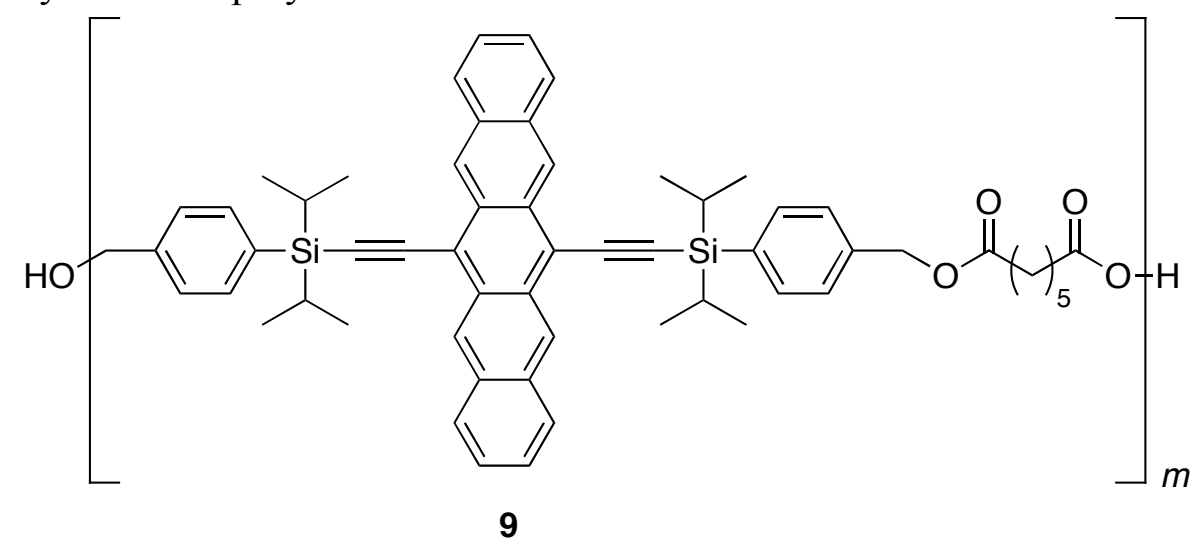

To a solution of $1\left(0.205 \mathrm{~g}, 0.261 \mathrm{mmol}\right.$, freshly recrystallized from $\mathrm{CH}_{2} \mathrm{Cl}_{2} /$ hexanes $)$ and DMAP $(0.159 \mathrm{~g}, 0.130 \mathrm{mmol})$ in dry $\mathrm{CH}_{2} \mathrm{Cl}_{2}(5 \mathrm{~mL})$ was added dropwise $0.50 \mathrm{~mL}(0.28 \mathrm{mmol})$ of a solution $0.56 \mathrm{M}$ pimeloyl chloride [pimeloyl chloride $(0.55 \mathrm{~g}, 0.46 \mathrm{~mL}, 2.8 \mathrm{mmol})$ dissolved in dry $\mathrm{CH}_{2} \mathrm{Cl}_{2}(5 \mathrm{~mL})$ under $\mathrm{N}_{2}$ atmosphere] over a period of $20 \mathrm{~min}$. The reaction was then allowed to stir for $2 \mathrm{~d}$ before being diluted into $\mathrm{CH}_{2} \mathrm{Cl}_{2}(50 \mathrm{~mL})$ and satd. aq. $\mathrm{NaHCO}_{3}(100 \mathrm{~mL})$. The mixture was separated and the organic phase was washed with $5 \%$ aq. $\mathrm{NaHCO}_{3}(3 \times 100$ $\mathrm{mL})$, satd. aq. $\mathrm{NaCl}(150 \mathrm{~mL})$, dried $\left(\mathrm{MgSO}_{4}\right)$, and the solvent removed in vacuo. The residue was dissolved in $\mathrm{CH}_{2} \mathrm{Cl}_{2}(2 \mathrm{~mL})$, and precipitated by adding hexanes $(40 \mathrm{~mL})$ and cooling to -78 ${ }^{\circ} \mathrm{C}$. After filtering, the resulting solid was washed with hexanes $(3 \times 10 \mathrm{~mL})$ and redissolved in $\mathrm{CH}_{2} \mathrm{Cl}_{2}$. This solution was filtered through a fritted funnel to remove any undissolved solids, concentrated in vacuo, and the resulting solid dried under vacuum to afford polymer $9(0.202 \mathrm{~g}$, $85 \%)$ as a deep blue solid. UV-vis $\left(\mathrm{CH}_{2} \mathrm{Cl}_{2}\right) \lambda_{\max }: 271,309,330,440,552,594,645 \mathrm{~nm}$. IR $\left(\mathrm{CDCl}_{3}\right.$, cast) $3045(\mathrm{w}), 2943(\mathrm{~s}), 2890(\mathrm{~m}), 2863(\mathrm{~m}), 2136(\mathrm{~m}), 1738(\mathrm{~s}) \mathrm{cm}^{-1} .{ }^{1} \mathrm{H}$ NMR $(500$ $\mathrm{MHz}, \mathrm{CDCl}_{3}$ ): $\delta 9.32$ (bs, 4H), 7.96-7.90 (m, 4H), 7.88 (broad d, $\left.J=7.9 \mathrm{~Hz}, 4 \mathrm{H}\right), 7.42-7.35$ (m, $8 \mathrm{H}), 5.12$ (bs, 4H), 2.35 (t, $J=7.5 \mathrm{~Hz}, 4 \mathrm{H}), 1.69-1.60(\mathrm{~m}, 4 \mathrm{H}), 1.59-1.47$ (m, 4H), 1.36 (broad $\mathrm{d}, J=7.4 \mathrm{~Hz}, 12 \mathrm{H}), 1.39-1.31(2 \mathrm{H}$, this signal overlaps a broad doublet at 1.36 , see analogous pattern in dimer 14), $1.22(\mathrm{~d}, J=7.4 \mathrm{~Hz}, 12 \mathrm{H}) .{ }^{13} \mathrm{C}$ NMR $\left(125 \mathrm{MHz}, \mathrm{CDCl}_{3}\right): \delta 173.3,137.3$, 135.5, 133.2, 132.4, 130.7, 128.6, 127.4, 126.2, 118.2, 105.8, 105.3, 66.0, 34.0, 28.5, 24.5, 18.2, 18.1, 12.0. TGA: $\mathrm{T}_{\mathrm{d}} \approx 380^{\circ} \mathrm{C}$. DSC: decomposition, $370{ }^{\circ} \mathrm{C}$ (onset) and $385^{\circ} \mathrm{C}$ (peak). 
Synthesis of dimer $\mathbf{1 0}$.

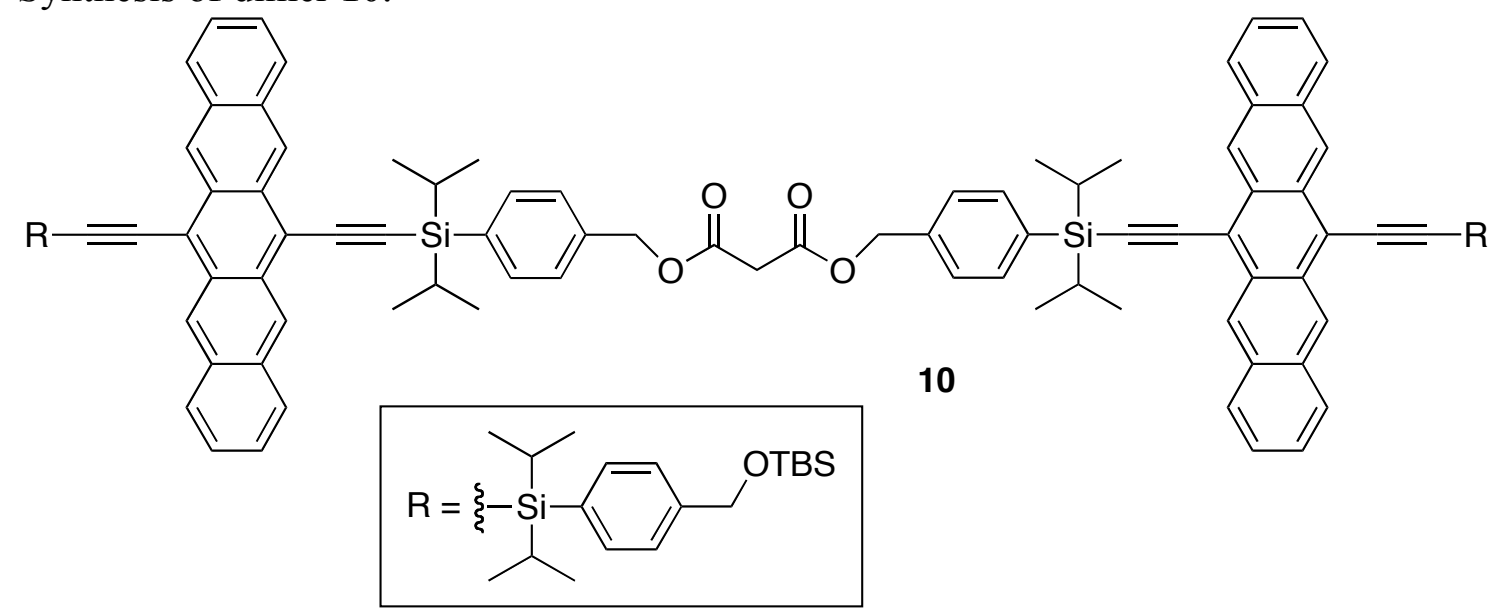

To a solution of $2(0.100 \mathrm{~g}, 0.114 \mathrm{mmol})$ and DMAP $(0.0282 \mathrm{~g}, 0.231 \mathrm{mmol})$ in dry $\mathrm{CH}_{2} \mathrm{Cl}_{2}(2$ $\mathrm{mL}$ ) was added dropwise $0.25 \mathrm{~mL}(0.057 \mathrm{mmol})$ of a solution $0.23 \mathrm{M}$ malonyl chloride [malonyl chloride $(0.16 \mathrm{~g}, 0.11 \mathrm{~mL}, 1.1 \mathrm{mmol})$ dissolved in dry $\mathrm{CH}_{2} \mathrm{Cl}_{2}(5 \mathrm{~mL})$ under $\mathrm{N}_{2}$ atmosphere] over a period of $5 \mathrm{~min}$. The reaction was allowed to stir for $10 \mathrm{~min}$ before being diluted with dry $\mathrm{CH}_{2} \mathrm{Cl}_{2}(20 \mathrm{~mL})$ and $5 \%$ aq. $\mathrm{NaHCO}_{3}(20 \mathrm{~mL})$. The mixture was separated and the organic phase was washed with satd. aq. $\mathrm{NaCl}(25 \mathrm{~mL})$, dried $\left(\mathrm{MgSO}_{4}\right)$, and the solvent removed in vacuo. Column chromatography (silica gel, $\left.\mathrm{CH}_{2} \mathrm{Cl}_{2}\right)$ afforded $10(0.0826 \mathrm{~g}, 80 \%)$ as a deep blue solid. $R_{\mathrm{f}}=0.79$ (4:1 $\mathrm{CH}_{2} \mathrm{Cl}_{2} /$ hexanes). UV-vis $\left(\mathrm{CH}_{2} \mathrm{Cl}_{2}\right) \lambda_{\max }(\varepsilon)$ : 271 (45 600), 309 (536 000), 330 (78 500), 440 (8 310), 552 (9 600), 594 (26 200), 645 (50 400) nm. IR (CDCl , cast) 3048 (w), 2952 (s), $2890(\mathrm{~m}), 2863(\mathrm{~s}), 2136(\mathrm{~m}), 1756(\mathrm{~m}), 1739(\mathrm{~m}) \mathrm{cm}^{-1} .{ }^{1} \mathrm{H}$ NMR $\left(500 \mathrm{MHz}, \mathrm{CDCl}_{3}\right): \delta 9.39$ (s, 4H), $9.36(\mathrm{~s}, 4 \mathrm{H}), 8.02-7.95(\mathrm{~m}, 8 \mathrm{H}), 7.92(\mathrm{~d}, J=8.1 \mathrm{~Hz}, 4 \mathrm{H}), 7.92(\mathrm{~d}, J=8.2 \mathrm{~Hz}, 4 \mathrm{H}), 7.48$ $7.40(\mathrm{~m}, 16 \mathrm{H}), 5.26(\mathrm{~s}, 4 \mathrm{H}), 4.83(\mathrm{~s}, 4 \mathrm{H}), 3.56(\mathrm{~s}, 2 \mathrm{H}), 1.58$ (septet, $J=7.4 \mathrm{~Hz}, 4 \mathrm{H}), 1.52$ (septet, $J=7.2 \mathrm{~Hz}, 4 \mathrm{H}), 1.42(\mathrm{~d}, J=7.3 \mathrm{~Hz}, 12 \mathrm{H}), 1.38(\mathrm{~d}, J=7.3 \mathrm{~Hz}, 12 \mathrm{H}), 1.29(\mathrm{~d}, J=7.3 \mathrm{~Hz}, 12 \mathrm{H})$, $1.24(\mathrm{~d}, J=7.2 \mathrm{~Hz}, 12 \mathrm{H}), 0.98(\mathrm{~s}, 18 \mathrm{H}), 0.14(\mathrm{~s}, 12 \mathrm{H}) .{ }^{13} \mathrm{C} \mathrm{NMR}\left(125 \mathrm{MHz}, \mathrm{CDCl}_{3}\right): \delta 166.3$, 142.9, 136.4, 135.6, 135.3, 133.8, 132.4, 132.4, 131.4, 130.7, 130.7, 128.7, 128.6, 127.6, 126.4, 126.2, 126.2, 126.2, 125.5, 118.5, 118.1, 106.0, 105.8, 105.6, 105.1, 67.2, 64.9, 41.6, 26.0, 18.4, $18.3,18.2,18.2,18.1,12.1,12.1,-5.3 .{ }^{13} \mathrm{C}$ NMR (APT, $\left.100 \mathrm{MHz}, \mathrm{CDCl}_{3}\right)$ : $\delta 166.3(\mathrm{C}), 142.9$ (C), $136.4(\mathrm{C}), 135.6(\mathrm{CH}), 135.3(\mathrm{CH}), 133.8(\mathrm{C}), 132.4(\mathrm{C}), 132.4(\mathrm{C}), 131.4(\mathrm{C}), 130.7(\mathrm{C})$, $130.7(\mathrm{C}), 128.7(\mathrm{CH}), 128.6(\mathrm{CH}), 127.6(\mathrm{CH}), 126.4(\mathrm{CH}), 126.2(\mathrm{CH}), 126.2(\mathrm{CH}), 126.2$ $(\mathrm{CH}), 125.5(\mathrm{CH}), 118.5(\mathrm{C}), 118.1(\mathrm{C}), 106.0(\mathrm{C}), 105.8(\mathrm{C}), 105.6(\mathrm{C}), 105.1(\mathrm{C}), 67.2\left(\mathrm{CH}_{2}\right)$, 64.9 $\left(\mathrm{CH}_{2}\right), 41.6\left(\mathrm{CH}_{2}\right), 26.0\left(\mathrm{CH}_{3}\right), 18.4(\mathrm{C}), 18.3\left(\mathrm{CH}_{3}\right), 18.2\left(\mathrm{CH}_{3}\right), 18.2\left(\mathrm{CH}_{3}\right), 18.1\left(\mathrm{CH}_{3}\right)$, $12.1(\mathrm{CH}), 12.1(\mathrm{CH}),-5.3\left(\mathrm{CH}_{3}\right)$. ESI MS $m / z 1852.9\left([\mathrm{M}+\mathrm{Na}]^{+}, 100\right)$. TGA: $\mathrm{T}_{\mathrm{d}} \approx 370{ }^{\circ} \mathrm{C}$.

DSC: decomposition, $370{ }^{\circ} \mathrm{C}$ (onset) and $390{ }^{\circ} \mathrm{C}$ (peak). 
Synthesis of dimer 11.

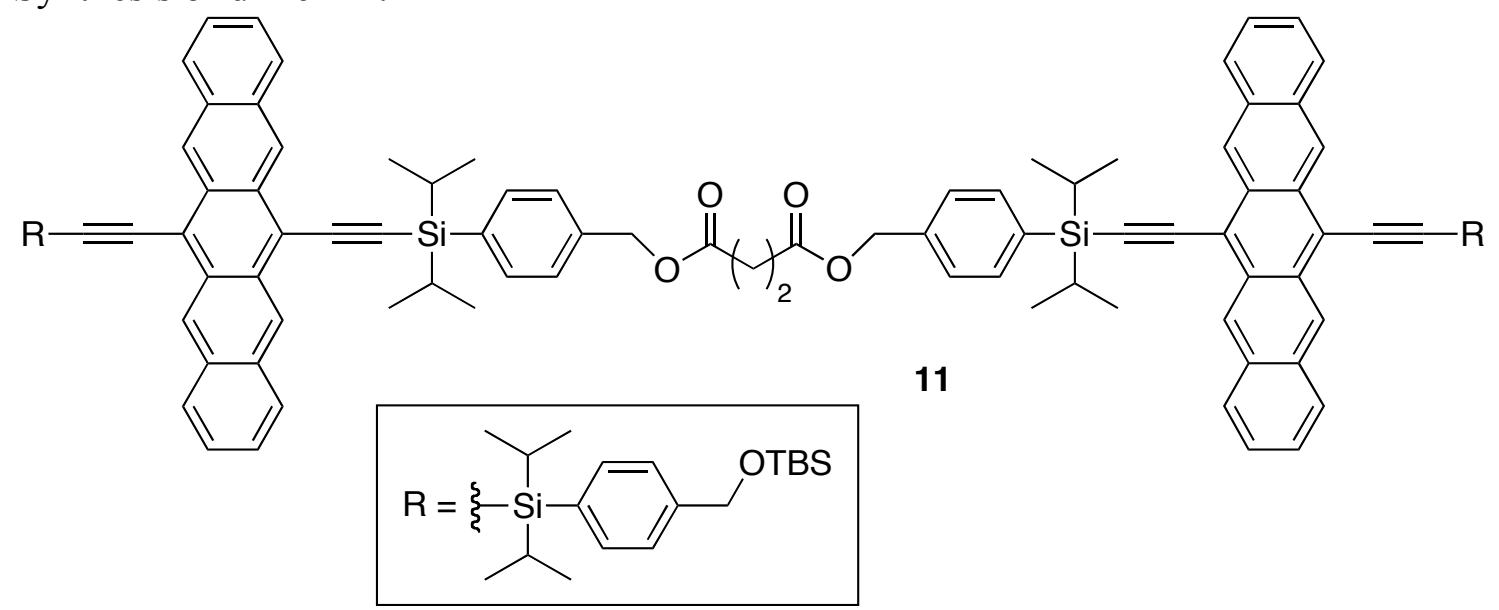

To a solution of $2(0.0749 \mathrm{~g}, 0.085 \mathrm{mmol})$ and DMAP $(0.0258 \mathrm{~g}, 0.211 \mathrm{mmol})$ in dry $\mathrm{CH}_{2} \mathrm{Cl}_{2}(2$ $\mathrm{mL})$ was added dropwise $0.25 \mathrm{~mL}(0.042 \mathrm{mmol})$ of a solution $0.17 \mathrm{M}$ succinyl chloride [succinyl chloride $(0.13 \mathrm{~g}, 0.094 \mathrm{~mL}, 0.85 \mathrm{mmol})$ dissolved in dry $\mathrm{CH}_{2} \mathrm{Cl}_{2}(5 \mathrm{~mL})$ under $\mathrm{N}_{2}$ atmosphere] over a period of $5 \mathrm{~min}$. The reaction was allowed to stir at $\mathrm{rt}$ for $3 \mathrm{~min}$ before adding dropwise another $0.25 \mathrm{~mL}(0.042 \mathrm{mmol})$ of $0.17 \mathrm{M}$ succinyl chloride solution followed by dry $\mathrm{CH}_{2} \mathrm{Cl}_{2}(5$ $\mathrm{mL})$. The reaction was allowed to stir for $5 \mathrm{~min}$ before being diluted with dry $\mathrm{CH}_{2} \mathrm{Cl}_{2}(20 \mathrm{~mL})$ and $5 \%$ aq. $\mathrm{NaHCO}_{3}(20 \mathrm{~mL})$. The mixture was separated and the organic phase was washed with satd. aq. $\mathrm{NaCl}(25 \mathrm{~mL})$, dried $\left(\mathrm{MgSO}_{4}\right)$, and the solvent removed in vacuo. Column chromatography (silica gel, $\left.\mathrm{CH}_{2} \mathrm{Cl}_{2}\right)$ afforded $11(0.0757 \mathrm{~g}, 97 \%)$ as a deep blue solid. $R_{\mathrm{f}}=0.65$ $\left(\mathrm{CH}_{2} \mathrm{Cl}_{2}\right)$. UV-vis $\left(\mathrm{CH}_{2} \mathrm{Cl}_{2}\right) \lambda_{\max }(\varepsilon)$ : 271 (45 800), 309 (508 000), 330 (73 400), 440 (7 500), 551 (8 660), 593 (24 400), 645 (47 100) nm. IR ( $\mathrm{CDCl}_{3}$, cast) 3048 (w), 2952 (s), 2863 (s), 2136 (m), $1740(\mathrm{~m}) \mathrm{cm}^{-1} .{ }^{1} \mathrm{H}$ NMR $\left(500 \mathrm{MHz}, \mathrm{CDCl}_{3}\right): \delta 9.40(\mathrm{~s}, 4 \mathrm{H}), 9.35(\mathrm{~s}, 4 \mathrm{H}), 8.03-7.97(\mathrm{~m}$, $8 \mathrm{H}), 7.93(\mathrm{~d}, J=8.0 \mathrm{~Hz}, 4 \mathrm{H}), 7.92(\mathrm{~d}, J=8.1 \mathrm{~Hz}, 4 \mathrm{H}), 7.47(\mathrm{~d}, J=8.2 \mathrm{~Hz}, 4 \mathrm{H}), 7.46(\mathrm{~d}, J=8.2$ $\mathrm{Hz}, 4 \mathrm{H}), 7.45-7.41(\mathrm{~m}, 8 \mathrm{H}), 5.21(\mathrm{~s}, 4 \mathrm{H}), 4.83(\mathrm{~s}, 4 \mathrm{H}), 2.78(\mathrm{~s}, 4 \mathrm{H}), 1.59$ (septet, $J=7.3 \mathrm{~Hz}$, 4H), 1.55 (septet, $J=7.2 \mathrm{~Hz}, 4 \mathrm{H}), 1.42$ (d, $J=7.3 \mathrm{~Hz}, 12 \mathrm{H}), 1.40$ (d, $J=7.3 \mathrm{~Hz}, 12 \mathrm{H}), 1.29$ (d, $J$ $=7.3 \mathrm{~Hz}, 12 \mathrm{H}), 1.26(\mathrm{~d}, J=7.3 \mathrm{~Hz}, 12 \mathrm{H}), 0.98(\mathrm{~s}, 18 \mathrm{H}), 0.14(\mathrm{~s}, 12 \mathrm{H}) .{ }^{13} \mathrm{C} \mathrm{NMR}(125 \mathrm{MHz}$, $\left.\mathrm{CDCl}_{3}\right): \delta 172.1,142.9,137.0,135.6,135.3,133.5,132.4,132.4,131.4,130.7,130.7,128.7$, $128.6,127.5,126.4,126.2,126.2,125.5,118.5,118.1,105.9,105.8,105.6,105.2,66.5,64.9$, $29.2,26.0,18.4,18.3,18.2,18.2,18.1,12.1,12.1,-5.3$ (one signal not observed). ${ }^{13} \mathrm{C} \mathrm{NMR}$ (APT, $\left.100 \mathrm{MHz}, \mathrm{CDCl}_{3}\right): \delta 172.1(\mathrm{C}), 142.9(\mathrm{C}), 137.0(\mathrm{C}), 135.6(\mathrm{CH}), 135.3(\mathrm{CH}), 133.5(\mathrm{C})$, $132.4(\mathrm{C}), 132.4(\mathrm{C}), 131.4(\mathrm{C}), 130.7(\mathrm{C}), 130.7(\mathrm{C}), 128.7(\mathrm{CH}), 128.6(\mathrm{CH}), 127.5(\mathrm{CH})$, $126.4(\mathrm{CH}), 126.2(\mathrm{CH}), 126.2(\mathrm{CH}), 125.5(\mathrm{CH}), 118.5(\mathrm{C}), 118.1(\mathrm{C}), 105.9(\mathrm{C}), 105.8(\mathrm{C})$, $105.6(\mathrm{C}), 105.2(\mathrm{C}), 66.5\left(\mathrm{CH}_{2}\right), 64.9\left(\mathrm{CH}_{2}\right), 29.2\left(\mathrm{CH}_{2}\right), 26.0\left(\mathrm{CH}_{3}\right), 18.4(\mathrm{C}), 18.3\left(\mathrm{CH}_{3}\right), 18.2$ $\left(\mathrm{CH}_{3}\right), 18.2\left(\mathrm{CH}_{3}\right), 18.1\left(\mathrm{CH}_{3}\right), 12.1(\mathrm{CH}), 12.1(\mathrm{CH}),-5.3\left(\mathrm{CH}_{3}\right)$ (one signal not observed). ESI MS $m / z 1866.9\left([\mathrm{M}+\mathrm{Na}]^{+}, 100\right)$. TGA: $\mathrm{T}_{\mathrm{d}} \approx 390{ }^{\circ} \mathrm{C}$. DSC: decomposition, $380^{\circ} \mathrm{C}$ (onset) and $395^{\circ} \mathrm{C}$ (peak). 
Synthesis of dimer 12.

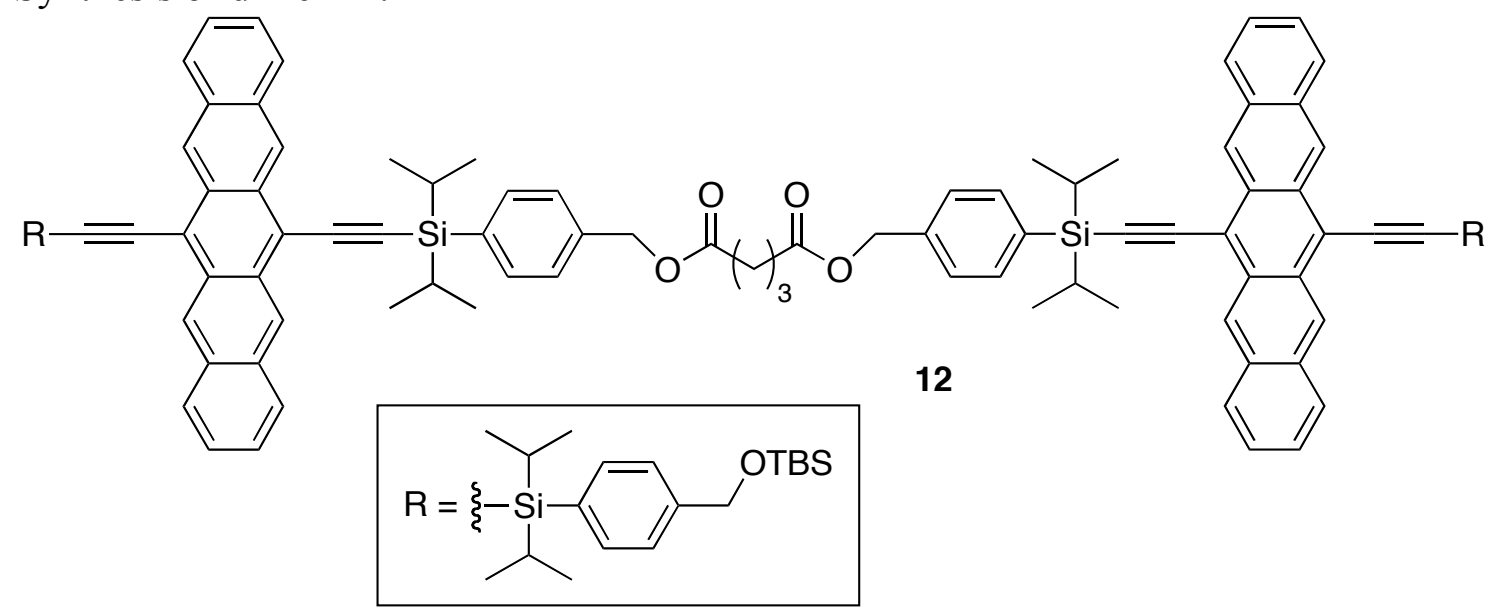

To a solution of $2(0.0500 \mathrm{~g}, 0.0567 \mathrm{mmol})$ and DMAP $(0.0182 \mathrm{~g}, 0.149 \mathrm{mmol})$ in dry $\mathrm{CH}_{2} \mathrm{Cl}_{2}(2$ $\mathrm{mL})$ was added dropwise $0.25 \mathrm{~mL}(0.028 \mathrm{mmol})$ of a solution $0.11 \mathrm{M}$ glutaryl chloride [glutaryl chloride $(0.096 \mathrm{~g}, 0.072 \mathrm{~mL}, 0.57 \mathrm{mmol})$ dissolved in dry $\mathrm{CH}_{2} \mathrm{Cl}_{2}(5 \mathrm{~mL})$ under $\mathrm{N}_{2}$ atmosphere] over a period of $5 \mathrm{~min}$. The reaction was allowed to stir at $\mathrm{rt}$ for $3 \mathrm{~min}$ before adding dropwise another $0.25 \mathrm{~mL}(0.028 \mathrm{mmol})$ of $0.11 \mathrm{M}$ glutaryl chloride solution followed by dry $\mathrm{CH}_{2} \mathrm{Cl}_{2}(5$ $\mathrm{mL})$. The reaction was allowed to stir for $5 \mathrm{~min}$ before being diluted with dry $\mathrm{CH}_{2} \mathrm{Cl}_{2}(20 \mathrm{~mL})$ and $5 \%$ aq. $\mathrm{NaHCO}_{3}(20 \mathrm{~mL})$. The mixture was separated and the organic phase was washed with satd. aq. $\mathrm{NaCl}(25 \mathrm{~mL})$, dried $\left(\mathrm{MgSO}_{4}\right)$, and the solvent removed in vacuo. Column chromatography (silica gel, $\left.\mathrm{CH}_{2} \mathrm{Cl}_{2}\right)$ afforded $12(0.0500 \mathrm{~g}, 95 \%)$ as a deep blue solid. $R_{\mathrm{f}}=0.64$ $\left(\mathrm{CH}_{2} \mathrm{Cl}_{2}\right)$. UV-vis $\left(\mathrm{CH}_{2} \mathrm{Cl}_{2}\right) \lambda_{\max }(\varepsilon)$ : 271 (46 500), 309 (547 000), 330 (79 800), 440 (8 410 ), 551 (9 690), 594 (26 600), 645 (51 100) nm. IR ( $\mathrm{CDCl}_{3}$, cast) 3047 (w), 2952 (s), 2863 (s), 2136 (m), $1740(\mathrm{~m}) \mathrm{cm}^{-1} .{ }^{1} \mathrm{H}$ NMR $\left(500 \mathrm{MHz}, \mathrm{CDCl}_{3}\right): \delta 9.37(\mathrm{~s}, 4 \mathrm{H}), 9.35(\mathrm{~s}, 4 \mathrm{H}), 8.00-7.94(\mathrm{~m}$, $8 \mathrm{H}), 7.90(\mathrm{~d}, J=8.1 \mathrm{~Hz}, 4 \mathrm{H}), 7.90(\mathrm{~d}, J=8.1 \mathrm{~Hz}, 4 \mathrm{H}), 7.46-7.38(\mathrm{~m}, 16 \mathrm{H}), 5.16(\mathrm{~s}, 4 \mathrm{H}), 4.81$ (s, 4H), 2.47 (t, $J=7.3 \mathrm{~Hz}, 4 \mathrm{H}), 2.04$ (quintet, $J=7.3 \mathrm{~Hz}, 2 \mathrm{H}$ ), 1.57 (septet, $J=7.3 \mathrm{~Hz}, 4 \mathrm{H}$ ), 1.55 (septet, $J=7.3 \mathrm{~Hz}, 4 \mathrm{H}), 1.40(\mathrm{~d}, J=7.1 \mathrm{~Hz}, 12 \mathrm{H}), 1.38(\mathrm{~d}, J=7.1 \mathrm{~Hz}, 12 \mathrm{H}), 1.27(\mathrm{~d}, J=7.3 \mathrm{~Hz}$, $12 \mathrm{H}), 1.25(\mathrm{~d}, J=7.3 \mathrm{~Hz}, 12 \mathrm{H}), 0.96(\mathrm{~s}, 18 \mathrm{H}), 0.12(\mathrm{~s}, 12 \mathrm{H}) .{ }^{13} \mathrm{C} \mathrm{NMR}\left(125 \mathrm{MHz}, \mathrm{CDCl}_{3}\right): \delta$ $172.7,142.9,137.1,135.6,135.3,133.4,132.4,132.4,131.4,130.7,130.7,128.7,128.6,127.5$, $126.4,126.2$, 126.2, 126.2, 125.5, 118.5, 118.1, 105.9, 105.8, 105.6, 105.2, 66.2, 64.9, 33.3, 26.0, 20.1, 18.4, 18.2, 18.2, 18.2, 18.1, 12.1, 12.1, -5.3. ${ }^{13} \mathrm{C}$ NMR (APT, $\left.100 \mathrm{MHz}, \mathrm{CDCl}_{3}\right): \delta 172.7$ (C), $142.9(\mathrm{C}), 137.1(\mathrm{C}), 135.6(\mathrm{CH}), 135.3(\mathrm{CH}), 133.4(\mathrm{C}), 132.4(\mathrm{C}), 132.4(\mathrm{C}), 131.4(\mathrm{C})$, $130.7(\mathrm{C}), 130.7(\mathrm{C}), 128.7(\mathrm{CH}), 128.6(\mathrm{CH}), 127.5(\mathrm{CH}), 126.4(\mathrm{CH}), 126.2(\mathrm{CH}), 126.2(\mathrm{CH})$, $126.2(\mathrm{CH}), 125.5(\mathrm{CH}), 118.5(\mathrm{C}), 118.1(\mathrm{C}), 105.9(\mathrm{C}), 105.8(\mathrm{C}), 105.6(\mathrm{C}), 105.2(\mathrm{C}), 66.2$ $\left(\mathrm{CH}_{2}\right), 64.9\left(\mathrm{CH}_{2}\right), 33.3\left(\mathrm{CH}_{2}\right), 26.0\left(\mathrm{CH}_{3}\right), 20.1\left(\mathrm{CH}_{2}\right), 18.4(\mathrm{C}), 18.2\left(\mathrm{CH}_{3}\right), 18.2\left(\mathrm{CH}_{3}\right), 18.2$ $\left(\mathrm{CH}_{3}\right), 18.1\left(\mathrm{CH}_{3}\right), 12.1(\mathrm{CH}), 12.1(\mathrm{CH}),-5.3\left(\mathrm{CH}_{3}\right)$. ESI MS $m / z 1880.9\left([\mathrm{M}+\mathrm{Na}]^{+}, 100\right)$. TGA: $\mathrm{T}_{\mathrm{d}} \approx 385^{\circ} \mathrm{C}$. DSC: decomposition, $360{ }^{\circ} \mathrm{C}$ (onset) and $390{ }^{\circ} \mathrm{C}$ (peak). 
Synthesis of dimer 13.

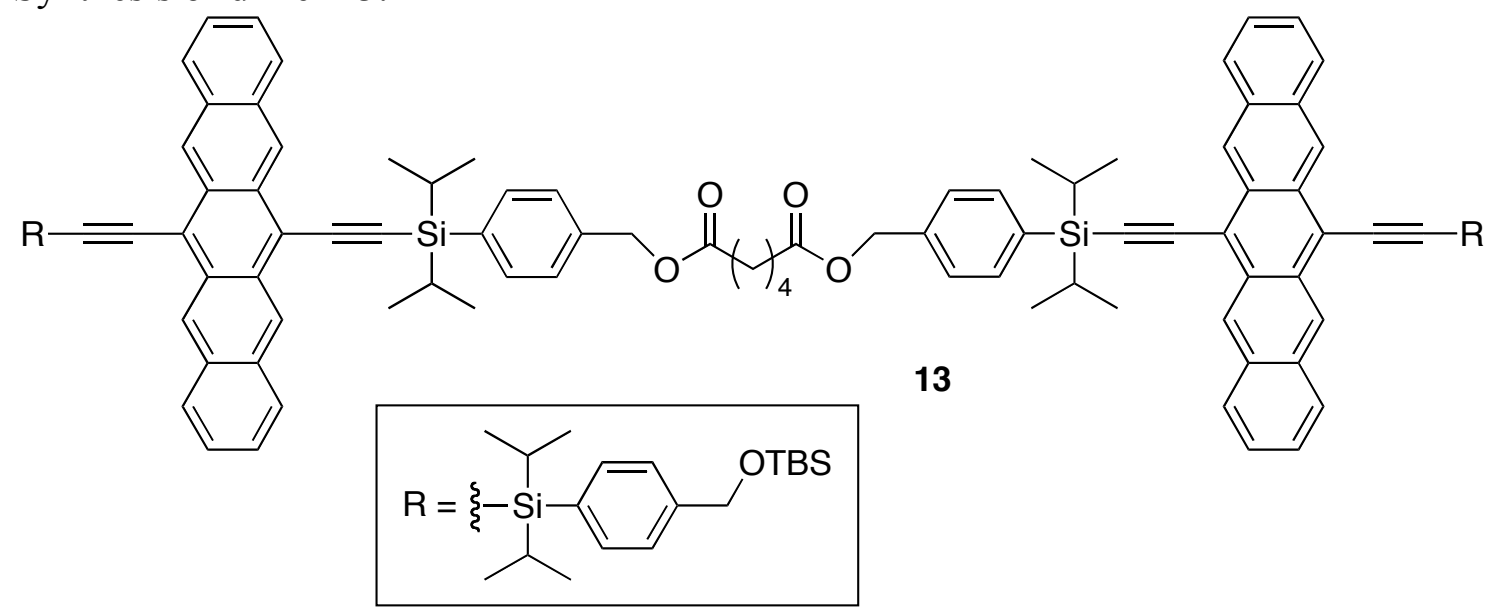

To a solution of $2(0.0624 \mathrm{~g}, 0.0708 \mathrm{mmol})$ and DMAP $(0.0216 \mathrm{~g}, 0.177 \mathrm{mmol})$ in dry $\mathrm{CH}_{2} \mathrm{Cl}_{2}(2$ $\mathrm{mL})$ was added dropwise $0.25 \mathrm{~mL}(0.035 \mathrm{mmol})$ of a solution $0.14 \mathrm{M}$ adipoyl chloride [adipoyl chloride $(0.13 \mathrm{~g}, 0.10 \mathrm{~mL}, 0.71 \mathrm{mmol})$ dissolved in dry $\mathrm{CH}_{2} \mathrm{Cl}_{2}(5 \mathrm{~mL})$ under $\mathrm{N}_{2}$ atmosphere over a period of $5 \mathrm{~min}$. The reaction was allowed to stir at $\mathrm{rt}$ for $3 \mathrm{~min}$ before adding dropwise another $0.25 \mathrm{~mL}(0.035 \mathrm{mmol})$ of $0.14 \mathrm{M}$ adipoyl chloride solution followed by dry $\mathrm{CH}_{2} \mathrm{Cl}_{2}(5$ $\mathrm{mL})$. The reaction was allowed to stir for $5 \mathrm{~min}$ before being diluted with dry $\mathrm{CH}_{2} \mathrm{Cl}_{2}(20 \mathrm{~mL})$ and $5 \%$ aq. $\mathrm{NaHCO}_{3}(20 \mathrm{~mL})$. The mixture was separated and the organic phase was washed with satd. aq. $\mathrm{NaCl}(25 \mathrm{~mL})$, dried $\left(\mathrm{MgSO}_{4}\right)$, and the solvent removed in vacuo. Column chromatography (silica gel, $\left.\mathrm{CH}_{2} \mathrm{Cl}_{2}\right)$ afforded $13(0.0579 \mathrm{~g}, 87 \%)$ as a deep blue solid. $R_{\mathrm{f}}=0.73$ $\left(\mathrm{CH}_{2} \mathrm{Cl}_{2}\right)$. UV-vis $\left(\mathrm{CH}_{2} \mathrm{Cl}_{2}\right) \lambda_{\max }(\varepsilon): 271$ (46 700), 309 (523 000), 329 (75 800), 440 (8 090), 550 (9 190), 593 (25 200), 644 (48 500) nm. IR ( $\mathrm{CDCl}_{3}$, cast) 3046 (w), 2952 (s), 2863 (s), 2136 (m), $1739(\mathrm{~m}) \mathrm{cm}^{-1} .{ }^{1} \mathrm{H}$ NMR $\left(500 \mathrm{MHz}, \mathrm{CDCl}_{3}\right): \delta 9.41(\mathrm{~s}, 4 \mathrm{H}), 9.39(\mathrm{~s}, 4 \mathrm{H}), 8.04-7.98(\mathrm{~m}$, $8 \mathrm{H}), 7.95(\mathrm{~d}, J=8.1 \mathrm{~Hz}, 4 \mathrm{H}), 7.93(\mathrm{~d}, J=7.4 \mathrm{~Hz}, 4 \mathrm{H}), 7.50-7.42(\mathrm{~m}, 16 \mathrm{H}), 5.20(\mathrm{~s}, 4 \mathrm{H}), 4.85$ (s, 4H), $2.44(\mathrm{~m}, 4 \mathrm{H}), 1.76(\mathrm{~m}, 4 \mathrm{H}), 1.60$ (septet, $J=7.3 \mathrm{~Hz}, 4 \mathrm{H}), 1.59$ (septet, $J=7.3 \mathrm{~Hz}, 4 \mathrm{H}), 1.43$ $(\mathrm{d}, J=7.2 \mathrm{~Hz}, 12 \mathrm{H}), 1.43(\mathrm{~d}, J=7.3 \mathrm{~Hz}, 12 \mathrm{H}), 1.30(\mathrm{~d}, J=7.3 \mathrm{~Hz}, 12 \mathrm{H}), 1.29$ (d, $J=7.3 \mathrm{~Hz}$, $12 \mathrm{H}), 0.99(\mathrm{~s}, 18 \mathrm{H}), 0.16(\mathrm{~s}, 12 \mathrm{H}) .{ }^{13} \mathrm{C} \mathrm{NMR}\left(125 \mathrm{MHz}, \mathrm{CDCl}_{3}\right): \delta 173.1,142.9,137.2,135.6$, $135.3,133.4,132.4,132.4,131.4,130.7,130.7,128.7,128.6,127.5,126.4,126.2,126.2,126.2$, $125.5,118.5,118.1,105.9,105.8,105.6,105.2,66.1,64.9,33.9,26.0,24.4,18.4,18.2,18.2$, 18.2, 18.1, 12.1, 12.1, -5.3. ${ }^{13} \mathrm{C}$ NMR (APT, $100 \mathrm{MHz}, \mathrm{CDCl}_{3}$ ): $\delta 173.1$ (C), 142.9 (C), 137.2 (C), $135.6(\mathrm{CH}), 135.3(\mathrm{CH}), 133.4(\mathrm{C}), 132.4(\mathrm{C}), 132.4(\mathrm{C}), 131.4(\mathrm{C}), 130.7(\mathrm{C}), 130.7(\mathrm{C})$, $128.7(\mathrm{CH}), 128.6(\mathrm{CH}), 127.5(\mathrm{CH}), 126.4(\mathrm{CH}), 126.2(\mathrm{CH}), 126.2(\mathrm{CH}), 125.5(\mathrm{CH}), 118.5$ (C), $118.1(\mathrm{C}), 105.9(\mathrm{C}), 105.8(\mathrm{C}), 105.6(\mathrm{C}), 105.2(\mathrm{C}), 66.1\left(\mathrm{CH}_{2}\right), 64.9\left(\mathrm{CH}_{2}\right), 33.9\left(\mathrm{CH}_{2}\right)$, $26.0\left(\mathrm{CH}_{3}\right), 24.4\left(\mathrm{CH}_{2}\right), 18.4(\mathrm{C}), 18.2\left(\mathrm{CH}_{3}\right), 18.2\left(\mathrm{CH}_{3}\right), 18.2\left(\mathrm{CH}_{3}\right), 18.1\left(\mathrm{CH}_{3}\right), 12.1(\mathrm{CH})$, $12.1(\mathrm{CH}),-5.3\left(\mathrm{CH}_{3}\right)$ (one signal not observed). ESI MS $m / z 1894.9\left([\mathrm{M}+\mathrm{Na}]^{+}, 100\right)$. TGA: $\mathrm{T}_{\mathrm{d}}$ $\approx 400{ }^{\circ} \mathrm{C}$. DSC (heating rate of $20{ }^{\circ} \mathrm{C} / \mathrm{min}$ ): decomposition, $370{ }^{\circ} \mathrm{C}$ (onset) and $405^{\circ} \mathrm{C}$ (peak). 
Synthesis of dimer 14.

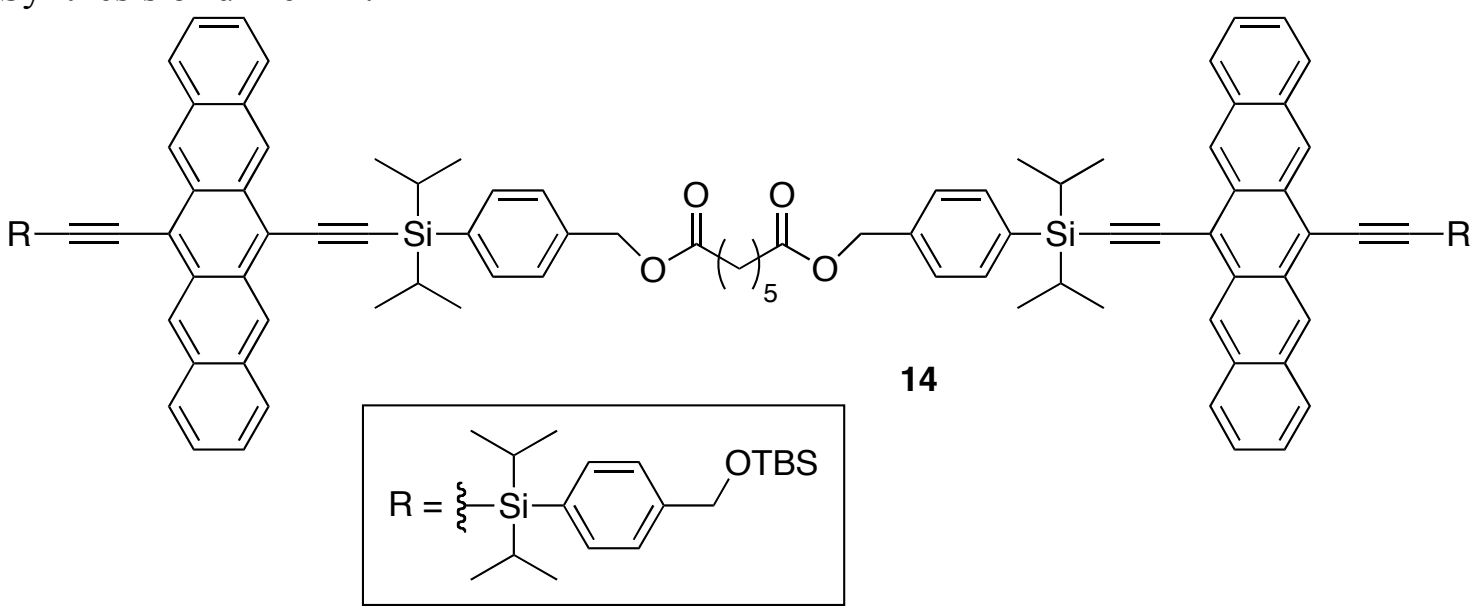

To a solution of $2(0.0401 \mathrm{~g}, 0.0455 \mathrm{mmol})$ and DMAP $(0.0144 \mathrm{~g}, 0.118 \mathrm{mmol})$ in dry $\mathrm{CH}_{2} \mathrm{Cl}_{2}(2$ $\mathrm{mL})$ was added dropwise $0.25 \mathrm{~mL}(0.023 \mathrm{mmol})$ of a solution $0.091 \mathrm{M}$ pimeloyl chloride [pimeloyl chloride $(0.090 \mathrm{~g}, 0.074 \mathrm{~mL}, 0.45 \mathrm{mmol})$ dissolved in dry $\mathrm{CH}_{2} \mathrm{Cl}_{2}(5 \mathrm{~mL})$ under $\mathrm{N}_{2}$ atmosphere] over a period of $5 \mathrm{~min}$. The reaction was allowed to stir at $\mathrm{rt}$ for $3 \mathrm{~min}$ before adding dropwise another $0.25 \mathrm{~mL}(0.023 \mathrm{mmol})$ of $0.091 \mathrm{M}$ pimeloyl chloride solution followed by dry $\mathrm{CH}_{2} \mathrm{Cl}_{2}(5 \mathrm{~mL})$. The reaction was allowed to stir for $5 \mathrm{~min}$ before being diluted with dry $\mathrm{CH}_{2} \mathrm{Cl}_{2}$ $(20 \mathrm{~mL})$ and $5 \%$ aq. $\mathrm{NaHCO}_{3}(20 \mathrm{~mL})$. The mixture was separated and the organic phase was washed with satd. aq. $\mathrm{NaCl}(25 \mathrm{~mL})$, dried $\left(\mathrm{MgSO}_{4}\right)$, and the solvent removed in vacuo. Column chromatography (silica gel, $\left.\mathrm{CH}_{2} \mathrm{Cl}_{2}\right)$ afforded $14(0.0400 \mathrm{~g}, 93 \%)$ as a deep blue solid. $R_{\mathrm{f}}=0.69$ $\left(\mathrm{CH}_{2} \mathrm{Cl}_{2}\right)$. UV-vis $\left(\mathrm{CH}_{2} \mathrm{Cl}_{2}\right) \lambda_{\max }(\varepsilon)$ : 271 (46 400), 309 (540 000), 330 (78 800), 440 (8 340), 552 (9 620), 594 (26 300), 645 (50 600) nm. IR ( $\mathrm{CDCl}_{3}$, cast) 3047 (w), 2941 (s), 2863 (s), 2137 (m), 1739 (s). ${ }^{1} \mathrm{H}$ NMR (500 MHz, $\left.\mathrm{CDCl}_{3}\right): \delta 9.38(\mathrm{~s}, 4 \mathrm{H}), 9.35(\mathrm{~s}, 4 \mathrm{H}), 8.01-7.95(\mathrm{~m}, 8 \mathrm{H}), 7.91$ $(\mathrm{d}, J=8.0 \mathrm{~Hz}, 4 \mathrm{H}), 7.90(\mathrm{~d}, J=8.0 \mathrm{~Hz}, 4 \mathrm{H}), 7.48-7.39(\mathrm{~m}, 16 \mathrm{H}), 5.16(\mathrm{~s}, 4 \mathrm{H}), 4.82(\mathrm{~s}, 4 \mathrm{H}), 2.38$ (t, $J=7.6 \mathrm{~Hz}, 4 \mathrm{H}$ ), 1.69 (quintet, $J=7.6 \mathrm{~Hz}, 4 \mathrm{H}$ ), 1.57 (septet, $J=7.1 \mathrm{~Hz}, 4 \mathrm{H}$ ), 1.56 (septet, $J=$ $7.1 \mathrm{~Hz}, 4 \mathrm{H}), 1.43-1.33\left(2 \mathrm{H}, \mathrm{CH}_{2}\right.$ identified by gCOSY $), 1.40(\mathrm{~d}, J=7.2 \mathrm{~Hz}, 12 \mathrm{H}), 1.39(\mathrm{~d}, J=$ $7.2 \mathrm{~Hz}, 12 \mathrm{H}), 1.27(\mathrm{~d}, J=6.3 \mathrm{~Hz}, 12 \mathrm{H}), 1.26(\mathrm{~d}, J=7.2 \mathrm{~Hz}, 12 \mathrm{H}), 0.96(\mathrm{~s}, 18 \mathrm{H}), 0.13(\mathrm{~s}, 12 \mathrm{H})$. gCOSY NMR $\left(500 \mathrm{MHz}, \mathrm{CDCl}_{3}\right): \delta 9.38 \leftrightarrow 8.01-7.95 ; 9.35 \leftrightarrow 8.01-7.95 ; 8.01-7.95 \leftrightarrow 9.38$, $9.35,7.48-7.39 ; 7.91 \leftrightarrow 7.48-7.39 ; 7.90 \leftrightarrow 7.48-7.39 ; 7.48-7.39 \leftrightarrow 8.01-7.95,7.91,7.90,5.16$, $4.82 ; 5.20 \leftrightarrow 7.48-7.39 ; 4.82 \leftrightarrow 7.48-7.39 ; 2.38 \leftrightarrow 1.69 ; 1.69 \leftrightarrow 2.38,1.43-1.33 ; 1.57 \leftrightarrow 1.40$, $1.27 ; 1.56 \leftrightarrow 1.39,1.26 ; 1.43-1.33 \leftrightarrow 1.69 ; 1.40 \leftrightarrow 1.57 ; 1.39 \leftrightarrow 1.56 ; 1.27 \leftrightarrow 1.57 ; 1.26 \leftrightarrow$ 1.56. ${ }^{13} \mathrm{C}$ NMR $\left(100 \mathrm{MHz}, \mathrm{CDCl}_{3}\right): \delta 173.4,142.9,137.3,135.6,135.3,133.3,132.4,132.4$, $131.4,130.7,130.7,128.7,128.6,127.5,126.4,126.2,126.2,126.2,125.5,118.5,118.1,105.9$, 105.8, 105.6, 105.2, 66.0, 64.9, 34.1, 28.6, 26.0, 24.6, 18.4, 18.2, 18.2, 18.2, 18.1, 12.1, 12.1, 5.3. ${ }^{13} \mathrm{C}$ NMR (APT, $\left.100 \mathrm{MHz}, \mathrm{CDCl}_{3}\right): \delta 173.4(\mathrm{C}), 142.9(\mathrm{C}), 137.3(\mathrm{C}), 135.6(\mathrm{CH}), 135.3$ $(\mathrm{CH}), 133.3(\mathrm{C}), 132.4(\mathrm{C}), 132.4(\mathrm{C}), 131.4(\mathrm{C}), 130.7(\mathrm{C}), 130.7(\mathrm{C}), 128.7(\mathrm{CH}), 128.6(\mathrm{CH})$, $127.5(\mathrm{CH}), 126.4(\mathrm{CH}), 126.2(\mathrm{CH}), 126.2(\mathrm{CH}), 125.5(\mathrm{CH}), 118.5(\mathrm{C}), 118.1(\mathrm{C}), 105.9(\mathrm{C})$, $105.8(\mathrm{C}), 105.6(\mathrm{C}), 105.2(\mathrm{C}), 66.0\left(\mathrm{CH}_{2}\right), 64.9\left(\mathrm{CH}_{2}\right), 34.1\left(\mathrm{CH}_{2}\right), 28.6\left(\mathrm{CH}_{2}\right), 26.0\left(\mathrm{CH}_{3}\right)$, $24.6\left(\mathrm{CH}_{2}\right), 18.3\left(\mathrm{CH}_{3}\right), 18.2\left(\mathrm{CH}_{3}\right), 18.2\left(\mathrm{CH}_{3}\right), 18.2\left(\mathrm{CH}_{3}\right), 12.1(\mathrm{CH}), 12.1(\mathrm{CH}),-5.3\left(\mathrm{CH}_{3}\right)$ (two signals not observed). ESI MS: $m / z 1909.9\left([\mathrm{M}+\mathrm{Na}]^{+}, 100\right)$. TGA: $\mathrm{T}_{\mathrm{d}} \approx 385^{\circ} \mathrm{C}$. DSC: decomposition, $375^{\circ} \mathrm{C}$ (onset) and $395^{\circ} \mathrm{C}$ (peak). 
Synthesis of dimer 15.

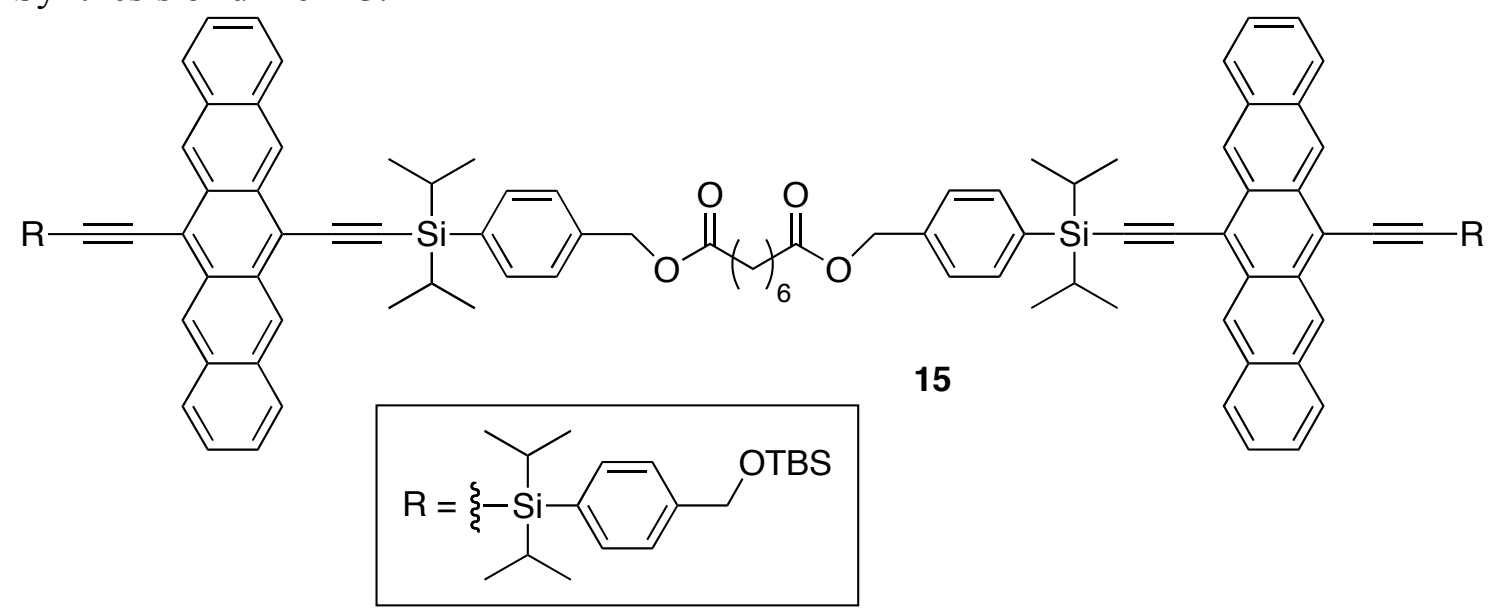

To a solution of $2(0.0610 \mathrm{~g}, 0.0692 \mathrm{mmol})$ and DMAP $(0.0213 \mathrm{~g}, 0.174 \mathrm{mmol})$ in dry $\mathrm{CH}_{2} \mathrm{Cl}_{2}(2$ $\mathrm{mL}$ ) was added dropwise $0.25 \mathrm{~mL}(0.035 \mathrm{mmol})$ of a solution $0.14 \mathrm{M}$ suberoyl chloride [suberoyl chloride $(0.15 \mathrm{~g}, 0.13 \mathrm{~mL}, 0.69 \mathrm{mmol})$ dissolved in dry $\mathrm{CH}_{2} \mathrm{Cl}_{2}(5 \mathrm{~mL})$ under $\mathrm{N}_{2}$ atmosphere] over a period of $5 \mathrm{~min}$. The reaction was allowed to stir at $\mathrm{rt}$ for $3 \mathrm{~min}$ before adding dropwise another $0.25 \mathrm{~mL}(0.035 \mathrm{mmol})$ of $0.14 \mathrm{M}$ suberoyl chloride solution followed by dry $\mathrm{CH}_{2} \mathrm{Cl}_{2}(5 \mathrm{~mL})$. The reaction was allowed to stir for $5 \mathrm{~min}$ before being diluted with dry $\mathrm{CH}_{2} \mathrm{Cl}_{2}$ $(20 \mathrm{~mL})$ and $5 \%$ aq. $\mathrm{NaHCO}_{3}(20 \mathrm{~mL})$. The mixture was separated and the organic phase was washed with satd. aq. $\mathrm{NaCl}(25 \mathrm{~mL})$, dried $\left(\mathrm{MgSO}_{4}\right)$, and the solvent removed in vacuo. Column chromatography (silica gel, $\left.\mathrm{CH}_{2} \mathrm{Cl}_{2}\right)$ afforded $15(0.0630 \mathrm{~g}, 96 \%)$ as a deep blue solid. $R_{\mathrm{f}}=0.78$ $\left(\mathrm{CH}_{2} \mathrm{Cl}_{2}\right)$. UV-vis $\left(\mathrm{CH}_{2} \mathrm{Cl}_{2}\right) \lambda_{\max }(\varepsilon)$ : 271 (44 800), 309 (514 000), 330 (73 000), 440 (7 360), 552 (8 570), 594 (24 300), 645 (47 100) nm. IR (CDCl 3 , cast) 3048 (w), 2943 (s), 2863 (s), 2136 $(\mathrm{m}), 1739(\mathrm{~s}) \mathrm{cm}^{-1} .{ }^{1} \mathrm{H}$ NMR $\left(500 \mathrm{MHz}, \mathrm{CDCl}_{3}\right): \delta 9.39(\mathrm{~s}, 4 \mathrm{H}), 9.37(\mathrm{~s}, 4 \mathrm{H}), 8.02-7.95(\mathrm{~m}, 8 \mathrm{H})$, $7.93(\mathrm{~d}, J=8.0 \mathrm{~Hz}, 4 \mathrm{H}), 7.91(\mathrm{~d}, J=7.2 \mathrm{~Hz}, 4 \mathrm{H}), 7.48-7.39(\mathrm{~m}, 16 \mathrm{H}), 5.17(\mathrm{~s}, 4 \mathrm{H}), 4.82(\mathrm{~s}, 4 \mathrm{H})$, 2.37 (t, $J=7.5 \mathrm{~Hz}, 4 \mathrm{H}), 1.71-1.63$ (m, 4H), 1.58 (septet, $J=7.5 \mathrm{~Hz}, 4 \mathrm{H}), 1.57$ (septet, $J=7.3$ $\mathrm{Hz}, 4 \mathrm{H}), 1.41(\mathrm{~d}, J=7.3 \mathrm{~Hz}, 12 \mathrm{H}), 1.41(\mathrm{~d}, J=7.2 \mathrm{~Hz}, 12 \mathrm{H}), 1.38-1.33(\mathrm{~m}, 4 \mathrm{H}), 1.28(\mathrm{~d}, J=7.3$ $\mathrm{Hz}, 12 \mathrm{H}), 1.27(\mathrm{~d}, J=7.3 \mathrm{~Hz}, 12 \mathrm{H}), 0.97(\mathrm{~s}, 18 \mathrm{H}), 0.13(\mathrm{~s}, 12 \mathrm{H}) .{ }^{13} \mathrm{C} \mathrm{NMR}\left(125 \mathrm{MHz}, \mathrm{CDCl}_{3}\right)$ : $\delta 173.5,142.9,137.4,135.6,135.3,133.3,132.4,132.4,131.4,130.7,130.7,128.7,128.6,127.4$, $126.4,126.2,126.2,126.2,125.5,118.5,118.1,105.9,105.8,105.6,105.2,66.0,64.9,34.2,28.7$, 26.0, 24.7, 18.4, 18.3, 18.2, 18.2, 18.1, 12.1, 12.1, -5.3. ${ }^{13} \mathrm{C}$ NMR (APT, $100 \mathrm{MHz}, \mathrm{CDCl}_{3}$ ): ठ $173.5(\mathrm{C}), 142.9(\mathrm{C}), 137.4(\mathrm{C}), 135.6(\mathrm{CH}), 135.3(\mathrm{CH}), 133.3(\mathrm{C}), 132.4(\mathrm{C}), 132.4(\mathrm{C})$, $131.4(\mathrm{C}), 130.7(\mathrm{C}), 130.7(\mathrm{C}), 128.7(\mathrm{CH}), 128.6(\mathrm{CH}), 127.4(\mathrm{CH}), 126.4(\mathrm{CH}), 126.2(\mathrm{CH})$, $126.2(\mathrm{CH}), 126.2(\mathrm{CH}), 125.5(\mathrm{CH}), 118.5(\mathrm{C}), 118.1(\mathrm{C}), 105.9(\mathrm{C}), 105.8(\mathrm{C}), 105.6(\mathrm{C})$, 105.2 (C), $66.0\left(\mathrm{CH}_{2}\right), 64.9\left(\mathrm{CH}_{2}\right), 34.2\left(\mathrm{CH}_{2}\right), 28.7\left(\mathrm{CH}_{2}\right), 26.0\left(\mathrm{CH}_{3}\right), 24.7\left(\mathrm{CH}_{2}\right), 18.4(\mathrm{C})$, $18.3\left(\mathrm{CH}_{3}\right), 18.2\left(\mathrm{CH}_{3}\right), 18.2\left(\mathrm{CH}_{3}\right), 18.1\left(\mathrm{CH}_{3}\right), 12.1(\mathrm{CH}), 12.1(\mathrm{CH}),-5.3\left(\mathrm{CH}_{3}\right)$. ESI MS $m / z$ $1922.9\left([\mathrm{M}+\mathrm{Na}]^{+}, 100\right)$. TGA (heating rate of $\left.20^{\circ} \mathrm{C} / \mathrm{min}\right): \mathrm{T}_{\mathrm{d}} \approx 400{ }^{\circ} \mathrm{C}$. DSC (heating rate of 20 ${ }^{\circ} \mathrm{C} / \mathrm{min}$ ): decomposition, $370{ }^{\circ} \mathrm{C}$ (onset) and $390{ }^{\circ} \mathrm{C}$ (peak). 
Synthesis of dimer 16.

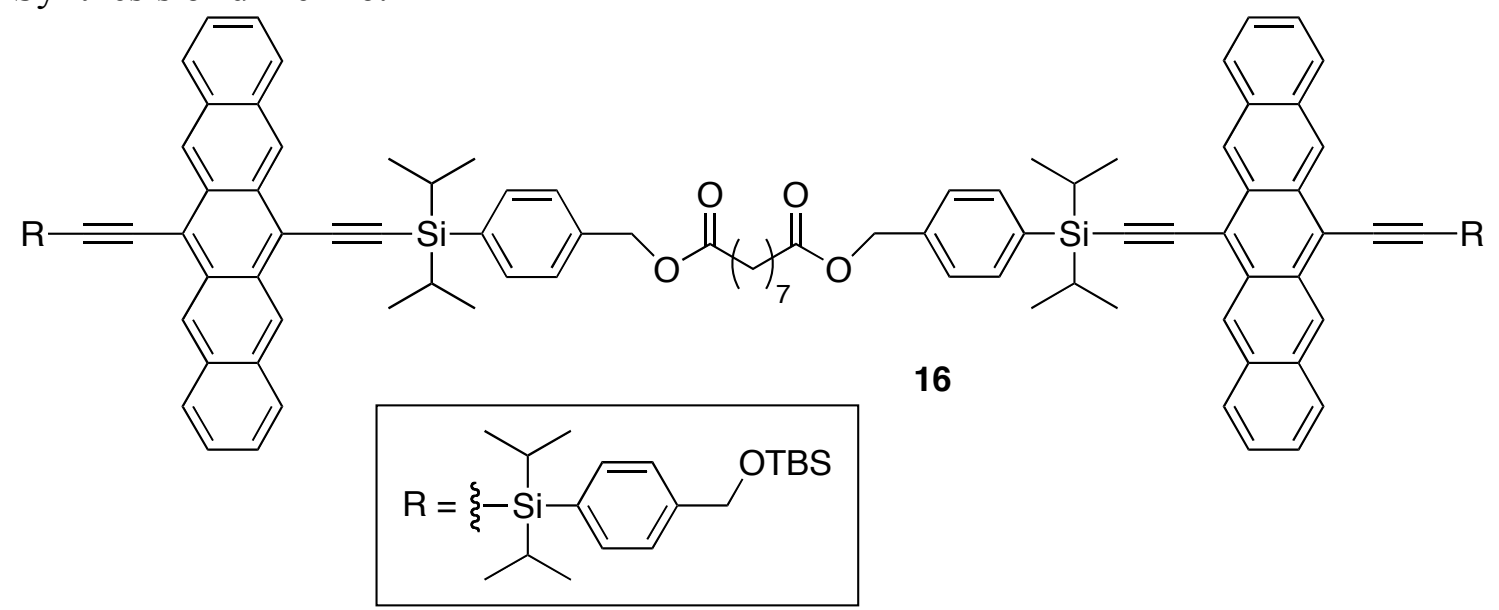

To a solution of $2(0.0381 \mathrm{~g}, 0.0432 \mathrm{mmol})$ and DMAP $(0.0131 \mathrm{~g}, 0.107 \mathrm{mmol})$ in dry $\mathrm{CH}_{2} \mathrm{Cl}_{2}(2$ $\mathrm{mL})$ was added dropwise $0.25 \mathrm{~mL}(0.022 \mathrm{mmol})$ of a solution $0.086 \mathrm{M}$ azelaic chloride [azelaic chloride $(0.097 \mathrm{~g}, 0.085 \mathrm{~mL}, 0.43 \mathrm{mmol})$ dissolved in dry $\mathrm{CH}_{2} \mathrm{Cl}_{2}(5 \mathrm{~mL})$ under $\mathrm{N}_{2}$ atmosphere] over a period of $5 \mathrm{~min}$. The reaction was allowed to stir at $\mathrm{rt}$ for $3 \mathrm{~min}$ before adding dropwise another $0.25 \mathrm{~mL}(0.022 \mathrm{mmol})$ of $0.086 \mathrm{M}$ azelaic chloride solution followed by dry $\mathrm{CH}_{2} \mathrm{Cl}_{2}(5$ $\mathrm{mL})$. The reaction was allowed to stir for $5 \mathrm{~min}$ before being diluted with dry $\mathrm{CH}_{2} \mathrm{Cl}_{2}(20 \mathrm{~mL})$ and $5 \%$ aq. $\mathrm{NaHCO}_{3}(20 \mathrm{~mL})$. The mixture was separated and the organic phase was washed with satd. aq. $\mathrm{NaCl}(25 \mathrm{~mL})$, dried $\left(\mathrm{MgSO}_{4}\right)$, and the solvent removed in vacuo. Column chromatography (silica gel, $\left.\mathrm{CH}_{2} \mathrm{Cl}_{2}\right)$ afforded $16(0.0354 \mathrm{~g}, 86 \%)$ as a deep blue solid. $R_{\mathrm{f}}=0.83$ $\left(\mathrm{CH}_{2} \mathrm{Cl}_{2}\right)$. UV-vis $\left(\mathrm{CH}_{2} \mathrm{Cl}_{2}\right) \lambda_{\max }(\varepsilon)$ : 271 (41 800), 309 (531 000), 330 (74 900), 440 (6 940), 552 (8 550), 594 (24 900), 645 (48 800) nm. IR ( $\mathrm{CDCl}_{3}$, cast) 3049 (w), 2941 (s), 2863 (s), 2136 (m), $1739(\mathrm{~s}) \mathrm{cm}^{-1} .{ }^{1} \mathrm{H}$ NMR $\left(500 \mathrm{MHz}, \mathrm{CDCl}_{3}\right): \delta 9.36(\mathrm{~s}, 4 \mathrm{H}), 9.34(\mathrm{~s}, 4 \mathrm{H}), 7.99-7.93(\mathrm{~m}, 8 \mathrm{H})$, $7.90(\mathrm{~d}, J=8.0 \mathrm{~Hz}, 4 \mathrm{H}), 7.89(\mathrm{~d}, J=7.6 \mathrm{~Hz}, 4 \mathrm{H}), 7.45-7.38(\mathrm{~m}, 16 \mathrm{H}), 5.15(\mathrm{~s}, 4 \mathrm{H}), 4.80(\mathrm{~s}, 4 \mathrm{H})$, $2.35(\mathrm{t}, J=7.6 \mathrm{~Hz}, 4 \mathrm{H}), 1.63$ (quintet, $J=6.9 \mathrm{~Hz}, 4 \mathrm{H}), 1.56$ (septet, $J=7.4 \mathrm{~Hz}, 8 \mathrm{H}), 1.39(\mathrm{~d}, J=$ $7.3 \mathrm{~Hz}, 24 \mathrm{H}), 1.33-1.28(\mathrm{~m}, 6 \mathrm{H}), 1.28(\mathrm{~d}, J=7.3 \mathrm{~Hz}, 12 \mathrm{H}), 1.25(\mathrm{~d}, J=7.6 \mathrm{~Hz}, 12 \mathrm{H}), 0.95$ (s, $18 \mathrm{H}), 0.11(\mathrm{~s}, 12 \mathrm{H}) .{ }^{13} \mathrm{C} \mathrm{NMR}\left(125 \mathrm{MHz}, \mathrm{CDCl}_{3}\right)$ : $\delta$ 173.6, 142.9, 137.4, 135.6, 135.3, 133.3, $132.4,132.4,131.4,130.7,130.7,128.7,128.6,127.4,126.4,126.2,126.2,126.2,125.5,118.5$, 118.1, 105.9, 105.8, 105.6, 105.2, 66.0, 64.9, 34.2, 28.9, 28.9, 26.0, 24.9, 18.4, 18.2, 18.2, 18.2, 18.1, 12.1, 12.1, -5.3. ${ }^{13} \mathrm{C}$ NMR (APT, $100 \mathrm{MHz} \mathrm{CDCl}_{3}$ ): $\delta 173.6(\mathrm{C}), 142.9(\mathrm{C}), 137.4(\mathrm{C})$, $135.6(\mathrm{CH}), 135.3(\mathrm{CH}), 133.3(\mathrm{C}), 132.4(\mathrm{C}), 132.4(\mathrm{C}), 131.4(\mathrm{C}), 130.7(\mathrm{C}), 130.7(\mathrm{C}), 128.7$ $(\mathrm{CH}), 128.6(\mathrm{CH}), 127.4(\mathrm{CH}), 126.4(\mathrm{CH}), 126.2(\mathrm{CH}), 126.2(\mathrm{CH}), 126.2(\mathrm{CH}), 125.5(\mathrm{CH})$, $118.5(\mathrm{C}), 118.1(\mathrm{C}), 105.9(\mathrm{C}), 105.8(\mathrm{C}), 105.6(\mathrm{C}), 105.2(\mathrm{C}), 66.0\left(\mathrm{CH}_{2}\right), 64.9\left(\mathrm{CH}_{2}\right), 34.2$ $\left(\mathrm{CH}_{2}\right), 28.9\left(\mathrm{CH}_{2}\right), 28.9\left(\mathrm{CH}_{2}\right), 26.0\left(\mathrm{CH}_{3}\right), 24.9\left(\mathrm{CH}_{2}\right), 18.4(\mathrm{C}), 18.2\left(\mathrm{CH}_{3}\right), 18.2\left(\mathrm{CH}_{3}\right), 18.2$ $\left(\mathrm{CH}_{3}\right), 18.1\left(\mathrm{CH}_{3}\right), 12.1(\mathrm{CH}), 12.1(\mathrm{CH}),-5.3\left(\mathrm{CH}_{3}\right)$. ESI MS $m / z 1937.0\left([\mathrm{M}+\mathrm{Na}]^{+}, 100\right)$. TGA: $\mathrm{T}_{\mathrm{d}} \approx 385^{\circ} \mathrm{C}$. DSC: decomposition, $380{ }^{\circ} \mathrm{C}$ (onset) and $395^{\circ} \mathrm{C}$ (peak). 
Synthesis of dimer 17.

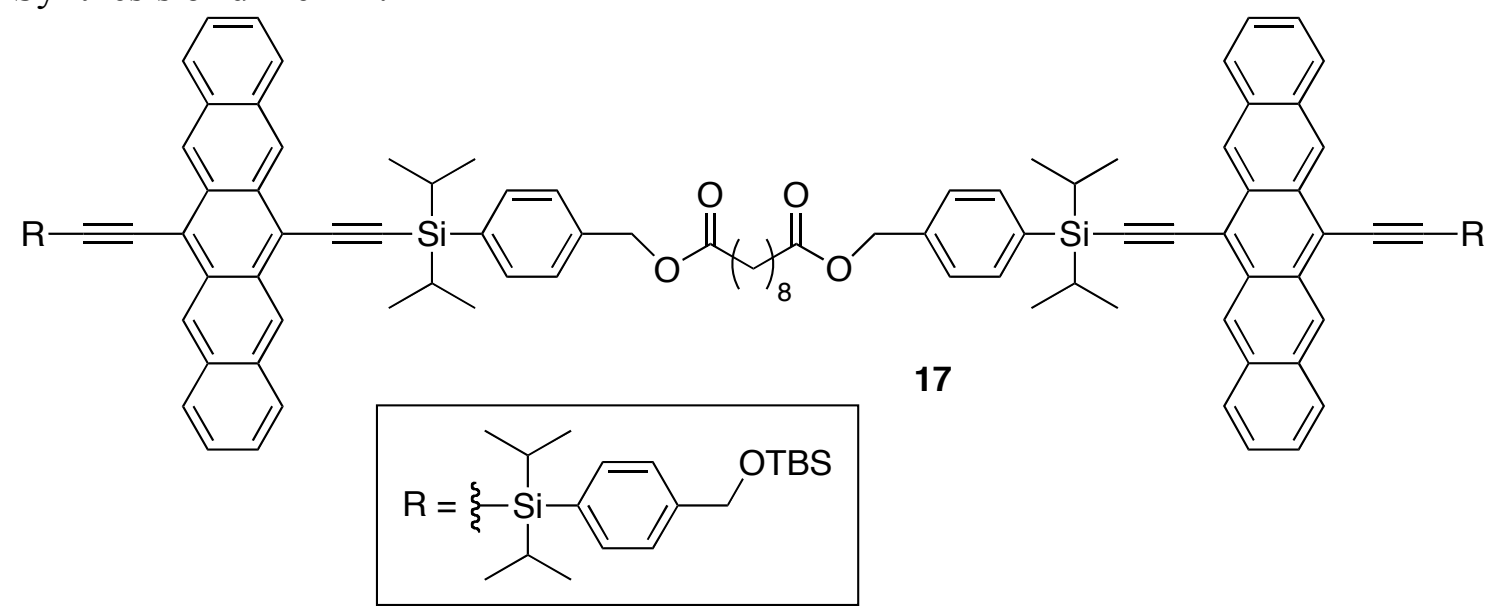

To a solution of $2(0.0755 \mathrm{~g}, 0.0857 \mathrm{mmol})$ and DMAP $(0.0511 \mathrm{~g}, 0.418 \mathrm{mmol})$ in dry $\mathrm{CH}_{2} \mathrm{Cl}_{2}(3$ $\mathrm{mL})$ was added dropwise $0.25 \mathrm{~mL}(0.043 \mathrm{mmol})$ of a solution $0.17 \mathrm{M}$ sebacoyl chloride [sebacoyl chloride $(0.20 \mathrm{~g}, 0.18 \mathrm{~mL}, 0.86 \mathrm{mmol})$ dissolved in dry $\mathrm{CH}_{2} \mathrm{Cl}_{2}(5 \mathrm{~mL})$ under $\mathrm{N}_{2}$ atmosphere] over a period of $5 \mathrm{~min}$. The reaction was allowed to stir at $\mathrm{rt}$ for $3 \mathrm{~min}$ before adding dropwise another $0.25 \mathrm{~mL}(0.043 \mathrm{mmol})$ of $0.17 \mathrm{M}$ sebacoyl chloride solution followed by dry $\mathrm{CH}_{2} \mathrm{Cl}_{2}(5 \mathrm{~mL})$. The reaction was allowed to stir for $5 \mathrm{~min}$ before being diluted with dry $\mathrm{CH}_{2} \mathrm{Cl}_{2}$ $(20 \mathrm{~mL})$ and $5 \%$ aq. $\mathrm{NaHCO}_{3}(20 \mathrm{~mL})$. The mixture was separated and the organic phase was washed with satd. aq. $\mathrm{NaCl}(25 \mathrm{~mL})$, dried $\left(\mathrm{MgSO}_{4}\right)$, and the solvent removed in vacuo. Column chromatography (silica gel, $\left.\mathrm{CH}_{2} \mathrm{Cl}_{2}\right)$ afforded $17(0.0652 \mathrm{~g}, 79 \%)$ as a deep blue solid. $R_{\mathrm{f}}=0.80$ $\left(\mathrm{CH}_{2} \mathrm{Cl}_{2}\right)$. UV-vis $\left(\mathrm{CH}_{2} \mathrm{Cl}_{2}\right) \lambda_{\max }(\varepsilon)$ : 271 (40 200), 309 (512 000), 330 (72 100), 440 (6 690), 552 (8 240), 594 (24 000), 645 (47 000) nm. IR ( $\mathrm{CDCl}_{3}$, cast) 3047 (w), 2941 (s), 2862 (s), 2136 (m), $1739(\mathrm{~s}) \mathrm{cm}^{-1} .{ }^{1} \mathrm{H}$ NMR $\left(500 \mathrm{MHz}, \mathrm{CDCl}_{3}\right): \delta 9.37(\mathrm{~s}, 4 \mathrm{H}), 9.35(\mathrm{~s}, 4 \mathrm{H}), 8.00-7.94(\mathrm{~m}, 8 \mathrm{H})$, $7.91(\mathrm{~d}, J=8.3 \mathrm{~Hz}, 4 \mathrm{H}), 7.90(\mathrm{~d}, J=8.3 \mathrm{~Hz}, 4 \mathrm{H}), 7.46-7.38(\mathrm{~m}, 16 \mathrm{H}), 5.16(\mathrm{~s}, 4 \mathrm{H}), 4.81(\mathrm{~s}, 4 \mathrm{H})$, 2.35 (t, $J=7.6 \mathrm{~Hz}, 4 \mathrm{H}), 1.63$ (quintet, $J=7.4 \mathrm{~Hz}, 4 \mathrm{H}$ ), 1.55 (septet, $J=7.3 \mathrm{~Hz}, 8 \mathrm{H}), 1.39$ (d, $J=$ $7.2 \mathrm{~Hz}, 24 \mathrm{H}), 1.31-1.24(\mathrm{~m}, 32 \mathrm{H}), 0.96(\mathrm{~s}, 18 \mathrm{H}), 0.12(\mathrm{~s}, 12 \mathrm{H}) .{ }^{13} \mathrm{C} \mathrm{NMR}\left(125 \mathrm{MHz}, \mathrm{CDCl}_{3}\right): \delta$ 173.6, 142.9, 137.4, 135.6, 135.3, 133.3, 132.4, 132.4, 131.4, 130.7, 130.7, 128.7, 128.6, 127.4, $126.4,126.2$, 126.2, 126.2, 125.5, 118.5, 118.1, 105.9, 105.8, 105.6, 105.2, 66.0, 64.9, 34.3, 29.1, 29.0, 26.0, 24.9, 18.4, 18.3, 18.2, 18.2, 18.2, 12.1, 12.1, -5.3. ${ }^{13} \mathrm{C}$ NMR (APT, $125 \mathrm{MHz}$, $\left.\mathrm{CDCl}_{3}\right): \delta 173.6(\mathrm{C}), 142.9(\mathrm{C}), 137.4(\mathrm{C}), 135.6(\mathrm{CH}), 135.3(\mathrm{CH}), 133.3(\mathrm{C}), 132.4(\mathrm{C}), 132.4$ $(\mathrm{C}), 131.4(\mathrm{C}), 130.7(\mathrm{C}), 130.7(\mathrm{C}), 128.7(\mathrm{CH}), 128.6(\mathrm{CH}), 127.4(\mathrm{CH}), 126.4(\mathrm{CH}), 126.2$ $(\mathrm{CH}), 126.2(\mathrm{CH}), 126.2(\mathrm{CH}), 125.5(\mathrm{CH}), 118.5(\mathrm{C}), 118.1(\mathrm{C}), 105.9(\mathrm{C}), 105.8(\mathrm{C}), 105.6$ (C), $105.2(\mathrm{C}), 66.0\left(\mathrm{CH}_{2}\right), 64.9\left(\mathrm{CH}_{2}\right), 34.3\left(\mathrm{CH}_{2}\right), 29.1\left(\mathrm{CH}_{2}\right), 29.0\left(\mathrm{CH}_{2}\right), 26.0\left(\mathrm{CH}_{3}\right), 24.9$ $\left(\mathrm{CH}_{2}\right), 18.4(\mathrm{C}), 18.3\left(\mathrm{CH}_{3}\right), 18.2\left(\mathrm{CH}_{3}\right), 18.2\left(\mathrm{CH}_{3}\right), 18.2\left(\mathrm{CH}_{3}\right), 12.1(\mathrm{CH}), 12.1(\mathrm{CH}),-5.3$ $\left(\mathrm{CH}_{3}\right)$. ESI MS $m / z 1951.0\left([\mathrm{M}+\mathrm{Na}]^{+}, 100\right)$. TGA: $\mathrm{T}_{\mathrm{d}} \approx 385^{\circ} \mathrm{C}$. DSC: decomposition, $375^{\circ} \mathrm{C}$ (onset) and $395^{\circ} \mathrm{C}$ (peak). 
Synthesis of acid $\mathbf{1 8 .}$

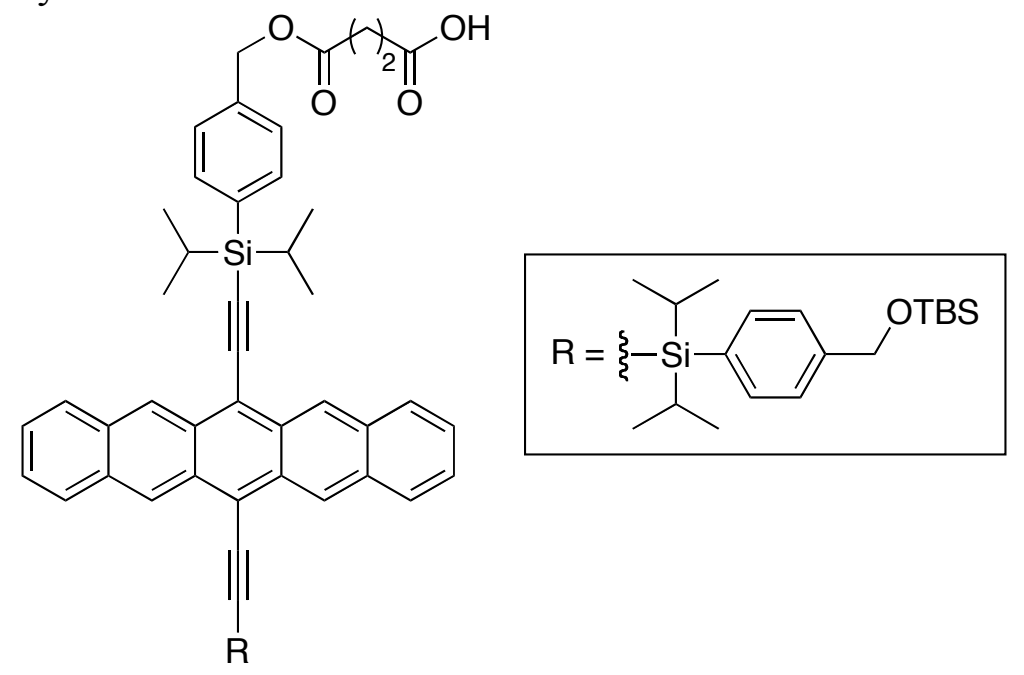

18

To a solution of $2(0.543 \mathrm{~g}, 0.616 \mathrm{mmol})$ and succinic anhydride $(0.066 \mathrm{~g}, 0.66 \mathrm{mmol})$ in dry $\mathrm{CH}_{2} \mathrm{Cl}_{2}(10 \mathrm{~mL})$ was added DMAP $(0.113 \mathrm{~g}, 0.925 \mathrm{mmol})$ under $\mathrm{N}_{2}$ atmosphere. The reaction was allowed to stir at $\mathrm{rt}$ for $1 \mathrm{~h}$ before adding additional succinic anhydride $(0.122 \mathrm{~g}, 1.21 \mathrm{mmol})$ and DMAP (0.116 g, $0.949 \mathrm{mmol})$. The reaction was stirred for an additional $1 \mathrm{~h}$ before being diluted with $\mathrm{CH}_{2} \mathrm{Cl}_{2}(100 \mathrm{~mL})$ and satd. aq. $\mathrm{NaHCO}_{3}(200 \mathrm{~mL})$. The mixture was separated and the organic phase was washed with satd. aq. $\mathrm{NaHCO}_{3}(200 \mathrm{~mL}), 5 \%$ aq. $\mathrm{NaHCO}_{3}(2 \times 200 \mathrm{~mL})$, satd. aq. $\mathrm{NaCl}(250 \mathrm{~mL})$, dried $\left(\mathrm{MgSO}_{4}\right)$, and the solvent removed in vacuo. This afforded 18 $(0.594 \mathrm{~g}, 98 \%)$ as a deep blue solid. IR $\left(\mathrm{CDCl}_{3}\right.$, cast) 3400-2400 (br, w) 3049 (m), 2952 (s), 2863 (s), 2136, (m) 1741 (s), 1714 (s) $\left.\mathrm{cm}^{-1} .{ }^{1} \mathrm{H} \mathrm{NMR} \mathrm{(500} \mathrm{MHz,} \mathrm{CDCl}_{3}\right): \delta 9.40$ (s, 2H), 9.37 (s, $2 \mathrm{H}), 8.02-7.96(\mathrm{~m}, 4 \mathrm{H}), 7.94(\mathrm{~d}, J=8.1 \mathrm{~Hz}, 2 \mathrm{H}), 7.90(\mathrm{~d}, J=8.2 \mathrm{~Hz}, 2 \mathrm{H}), 7.48-7.40(\mathrm{~m}, 8 \mathrm{H})$, $5.21(\mathrm{~s}, 2 \mathrm{H}), 4.83(\mathrm{~s}, 2 \mathrm{H}), 2.72-2.68(\mathrm{~m}, 4 \mathrm{H}), 1.58$ (septet, $J=7.3 \mathrm{~Hz}, 4 \mathrm{H}), 1.41(\mathrm{~m}, 12 \mathrm{H}), 1.28$ $(\mathrm{m}, 12 \mathrm{H}), 0.97(\mathrm{~s}, 9 \mathrm{H}), 0.14(\mathrm{~s}, 6 \mathrm{H}) .{ }^{13} \mathrm{C} \mathrm{NMR}\left(125 \mathrm{MHz}, \mathrm{CDCl}_{3}\right): \delta 178.0,171.9,142.9,137.0$, 135.6, 135.3, 133.5, 132.4, 132.4, 131.4, 130.7, 130.7, 128.7, 128.6, 127.5, 126.4, 126.2, 126.2, $125.5,118.5,118.1,105.9,105.8,105.6,105.2,66.5,64.9,29.7,28.8,26.0,18.4,18.2,18.2$, 18.2, 18.1, 12.1, 12.1, -5.3 (one signal not observed). ${ }^{13} \mathrm{C} \mathrm{NMR}$ (APT, $125 \mathrm{MHz}, \mathrm{CDCl}_{3}$ ):

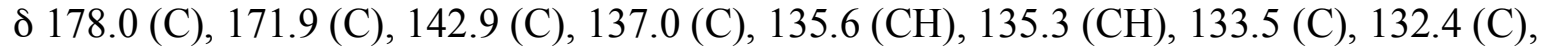
132.4 (C), $131.4(\mathrm{C}), 130.7(\mathrm{C}), 130.7(\mathrm{C}), 128.7(\mathrm{CH}), 128.6(\mathrm{CH}), 127.5(\mathrm{CH}), 126.4(\mathrm{CH})$, $126.2(\mathrm{CH}), 126.2(\mathrm{CH}), 125.5(\mathrm{CH}), 118.5(\mathrm{C}), 118.1(\mathrm{C}), 105.9(\mathrm{C}), 105.8(\mathrm{C}), 105.6(\mathrm{C})$, $105.2(\mathrm{C}), 66.5\left(\mathrm{CH}_{2}\right), 64.9\left(\mathrm{CH}_{2}\right), 29.7\left(\mathrm{CH}_{2}\right), 28.9\left(\mathrm{CH}_{2}\right), 26.0\left(\mathrm{CH}_{3}\right), 18.4(\mathrm{CH}), 18.2\left(\mathrm{CH}_{3}\right)$, $18.2\left(\mathrm{CH}_{3}\right), 18.2\left(\mathrm{CH}_{3}\right), 18.1\left(\mathrm{CH}_{3}\right), 12.1(\mathrm{CH}), 12.1(\mathrm{CH}),-5.3\left(\mathrm{CH}_{3}\right)$ (one signal not observed). ESI MS $m / z 1003.5\left([\mathrm{M}+\mathrm{Na}]^{+}, 100\right)$. ESI HRMS $m / z$ calcd. for $\mathrm{C}_{62} \mathrm{H}_{72} \mathrm{O}_{5} \mathrm{Si}_{3} \mathrm{Na}\left(\left[\mathrm{M}+\mathrm{Na}^{+}\right]\right)$ 1003.4580, found 1003.4576 . 
Synthesis of acid 19.

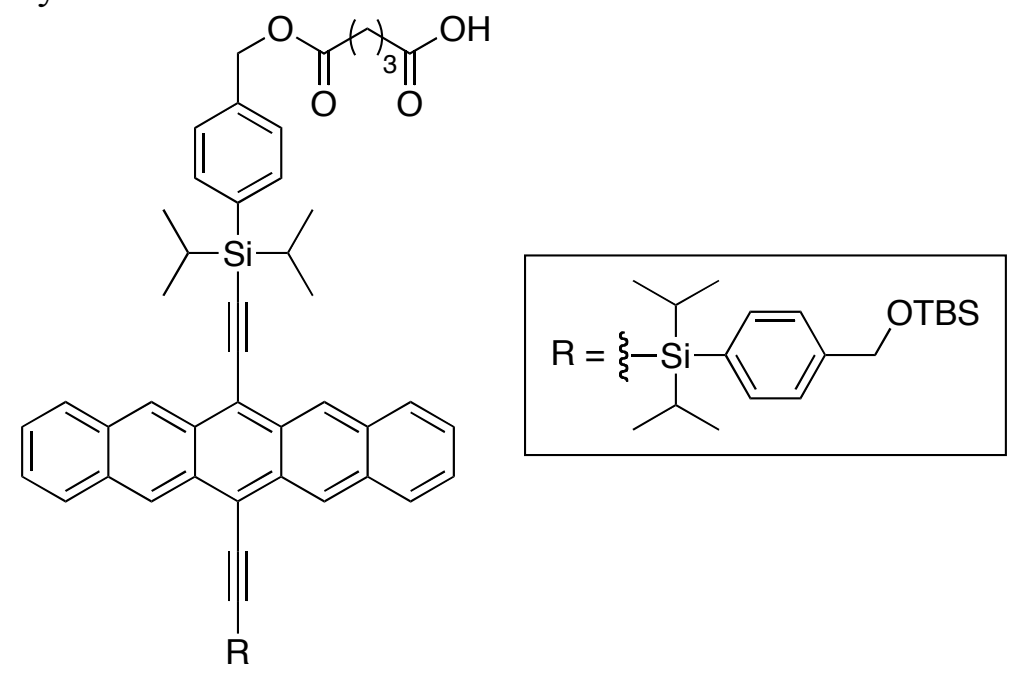

19

To a solution of $2(0.2966 \mathrm{~g}, 0.3365 \mathrm{mmol})$ and glutaric anhydride $(0.150 \mathrm{~g}, 1.31 \mathrm{mmol})$ in dry $\mathrm{CH}_{2} \mathrm{Cl}_{2}(7 \mathrm{~mL})$ was added DMAP $(0.158 \mathrm{~g}, 1.29 \mathrm{mmol})$ under $\mathrm{N}_{2}$ atmosphere. The reaction was allowed to stir at $\mathrm{rt}$ for $2 \mathrm{~h}$ before being diluted with $\mathrm{CH}_{2} \mathrm{Cl}_{2}(70 \mathrm{~mL})$ and satd. aq. $\mathrm{NaHCO}_{3}(200$ $\mathrm{mL})$. The mixture was separated and the organic phase was washed with satd. aq. $\mathrm{NaHCO}_{3}(200$ $\mathrm{mL}), 5 \%$ aq. $\mathrm{NaHCO}_{3}(2 \times 200 \mathrm{~mL})$, satd. aq. $\mathrm{NaCl}(250 \mathrm{~mL})$, dried $\left(\mathrm{MgSO}_{4}\right)$, and the solvent removed in vacuo. This afforded $19(0.3324 \mathrm{~g}, 99 \%)$ as a deep blue solid. IR $\left(\mathrm{CDCl}_{3}\right.$, cast $) 3048$ (w), 2952 (s), 2863 (s), 2136 (m), 1739 (s), 1710 (s) cm ${ }^{-1} .{ }^{1} \mathrm{H}$ NMR (500 MHz, CDCl 3 ): $\delta 9.40$ $(\mathrm{s}, 2 \mathrm{H}), 9.38(\mathrm{~s}, 2 \mathrm{H}), 8.02-7.97(\mathrm{~m}, 4 \mathrm{H}), 7.94(\mathrm{~d}, J=8.2 \mathrm{~Hz}, 2 \mathrm{H}), 7.92(\mathrm{~d}, J=7.7 \mathrm{~Hz}, 2 \mathrm{H}), 7.48-$ $7.41(\mathrm{~m}, 8 \mathrm{H}), 5.20(\mathrm{~s}, 2 \mathrm{H}), 4.83(\mathrm{~s}, 2 \mathrm{H}), 2.49(\mathrm{t}, J=7.4 \mathrm{~Hz}, 2 \mathrm{H}), 2.45(\mathrm{t}, J=7.3 \mathrm{~Hz}, 2 \mathrm{H}), 2.01$ (quintet, $J=7.3 \mathrm{~Hz}, 2 \mathrm{H}), 1.58$ (septet, $J=7.3 \mathrm{~Hz}, 4 \mathrm{H}), 1.42(\mathrm{~d}, J=7.3 \mathrm{~Hz}, 12 \mathrm{H}), 1.29$ (d, $J=7.3$ $\mathrm{Hz}, 12 \mathrm{H}), 0.98$ (s, 9H), $0.14(\mathrm{~s}, 6 \mathrm{H})$. gCOSY NMR (500 MHz, $\left.\mathrm{CDCl}_{3}\right): \delta 9.40 \leftrightarrow 8.02-7.97$; $9.38 \leftrightarrow 8.02-7.97 ; 8.02-7.97 \leftrightarrow 9.40,9.38 ; 7.94 \leftrightarrow 7.48-7.41 ; 7.92 \leftrightarrow 7.48-7.41 ; 7.48-7.41 \leftrightarrow$ $8.02-7.97,7.94,7.92,5.20,4.83 ; 5.20 \leftrightarrow 7.48-7.40 ; 4.83 \leftrightarrow 7.48-7.41 ; 2.49 \leftrightarrow 2.01 ; 2.45 \leftrightarrow$ $2.01 ; 2.01 \leftrightarrow 2.45,2.01 ; 1.58 \leftrightarrow 1.42,1.29 ; 1.42 \leftrightarrow 1.58 ; 1.29 \leftrightarrow 1.58 .{ }^{13} \mathrm{C} \mathrm{NMR}(125 \mathrm{MHz}$, $\mathrm{CDCl}_{3}$ ): $\delta 178.3$ (broad), 172.7, 142.9, 137.1, 135.6, 135.3, 133.5, 132.4, 132.4, 131.4, 130.7, $130.7,128.7,128.6,127.5,126.4,126.2,126.2$, 125.5, 118.5, 118.1, 105.9, 105.8, 105.6, 105.2, $66.2,64.9,33.2,29.7,26.0,19.9,18.4,18.2,18.2,18.1,18.1,12.1,12.1,-5.3$ (two signals not observed). ${ }^{13} \mathrm{C}$ NMR (APT, $\left.125 \mathrm{MHz}, \mathrm{CDCl}_{3}\right): \delta 172.7(\mathrm{C}), 142.9(\mathrm{C}), 137.1(\mathrm{C}), 135.6(\mathrm{CH})$, $135.3(\mathrm{CH}), 133.5(\mathrm{C}), 132.5(\mathrm{C}), 132.4(\mathrm{C}), 131.4(\mathrm{C}), 130.7(\mathrm{C}), 130.7(\mathrm{C}), 128.7(\mathrm{CH}), 128.6$ $(\mathrm{CH}), 127.5(\mathrm{CH}), 126.4(\mathrm{CH}), 126.2(\mathrm{CH}), 126.2(\mathrm{CH}), 125.5(\mathrm{CH}), 118.5(\mathrm{C}), 118.1(\mathrm{C}), 105.9$ (C), $105.8(\mathrm{C}), 105.6(\mathrm{C}), 105.2(\mathrm{C}), 66.2\left(\mathrm{CH}_{2}\right), 64.9\left(\mathrm{CH}_{2}\right), 33.2\left(\mathrm{CH}_{2}\right), 29.7\left(\mathrm{CH}_{2}\right), 26.0$ $\left(\mathrm{CH}_{3}\right), 19.9\left(\mathrm{CH}_{2}\right), 18.5(\mathrm{C}), 18.3\left(\mathrm{CH}_{3}\right), 18.2\left(\mathrm{CH}_{3}\right), 18.1\left(\mathrm{CH}_{3}\right), 18.1\left(\mathrm{CH}_{3}\right), 12.1(\mathrm{CH}), 12.1$ $(\mathrm{CH}),-5.3\left(\mathrm{CH}_{3}\right)$ (two signals not observed). ESI MS $m / z$ 993.5 ([M - H] $\left.]^{-}, 100\right)$. ESI HRMS $m / z$ calcd. for $\mathrm{C}_{63} \mathrm{H}_{73} \mathrm{O}_{5} \mathrm{Si}_{3}\left(\left[\mathrm{M}-\mathrm{H}^{+}\right]^{-}\right)$993.4771, found 993.4758 . 
Synthesis of trimer $\mathbf{2 0}$.

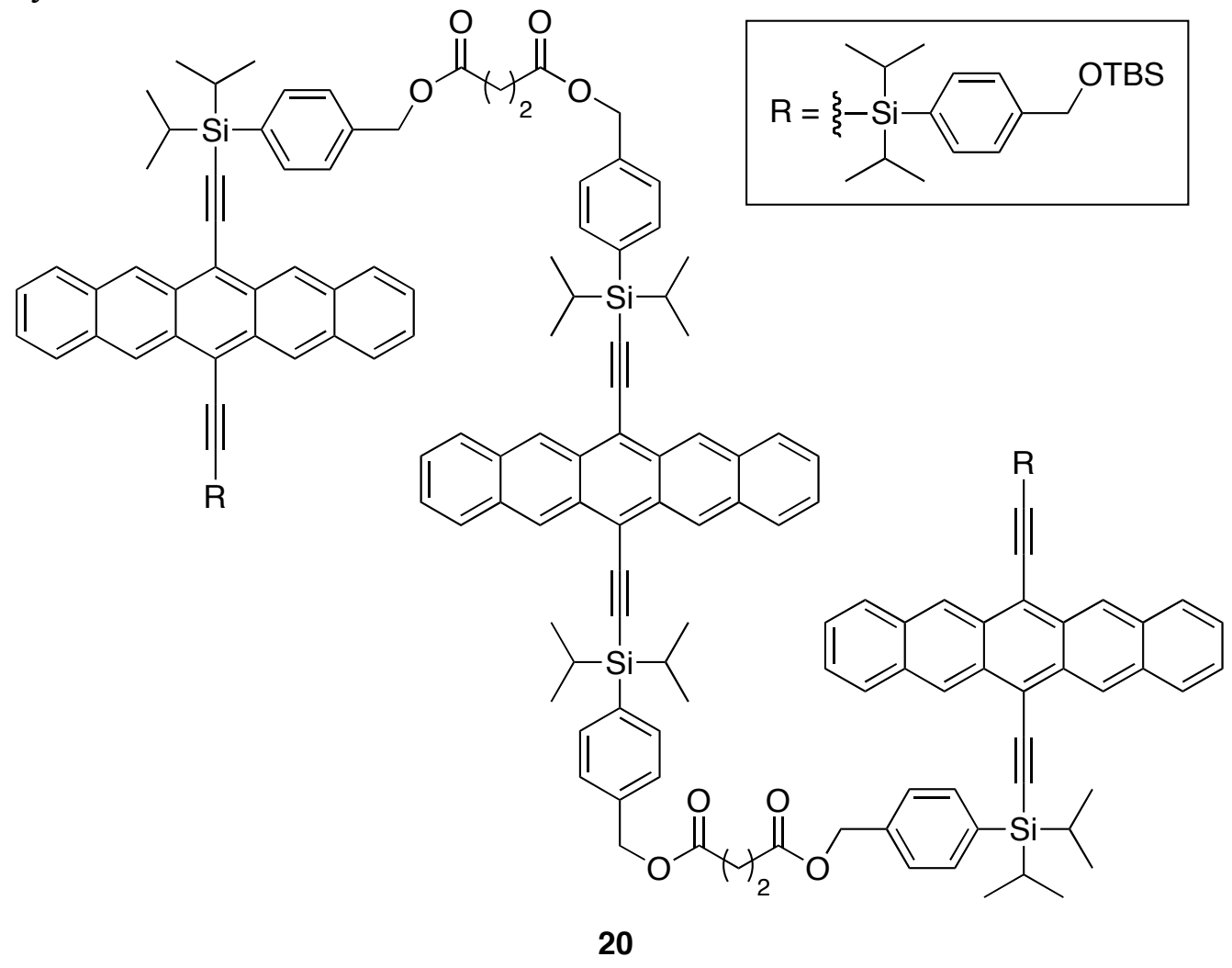

To a solution of $18(0.297 \mathrm{~g}, 0.302 \mathrm{mmol})$ and $\mathbf{1}(0.116 \mathrm{~g}, 0.151 \mathrm{mmol})$ in dry $\mathrm{CH}_{2} \mathrm{Cl}_{2}(10 \mathrm{~mL})$ was added DMAP $(0.076 \mathrm{~g}, 0.62 \mathrm{mmol})$ and DCC $(0.098 \mathrm{~g}, 0.47 \mathrm{mmol})$ under $\mathrm{N}_{2}$ atmosphere. The reaction was allowed to stir at $\mathrm{rt}$ for $1 \mathrm{~h}$ before adding additional DMAP $(0.029 \mathrm{~g}, 0.24$ $\mathrm{mmol})$ and DCC $(0.098 \mathrm{~g}, 0.47 \mathrm{mmol})$ and stirring for an additional $2 \mathrm{~h}$ before placing the flask in the fridge $\left(4{ }^{\circ} \mathrm{C}\right)$ overnight to allow for most of the dicyclohexylurea to precipitate. The reaction was filtered and the filtrate was diluted with $\mathrm{CH}_{2} \mathrm{Cl}_{2}(80 \mathrm{~mL})$ and satd. aq. $\mathrm{NaHCO}_{3}$ $(200 \mathrm{~mL})$. The mixture was separated and the organic phase was washed with satd. aq. $\mathrm{NaHCO}_{3}$ $(200 \mathrm{~mL}), 5 \%$ aq. $\mathrm{NaHCO}_{3}(2 \times 200 \mathrm{~mL})$, satd. aq. $\mathrm{NaCl}(250 \mathrm{~mL})$, dried $\left(\mathrm{MgSO}_{4}\right)$, and the solvent removed in vacuo. Column chromatography (silica gel, $\mathrm{CH}_{2} \mathrm{Cl}_{2}$ ) afforded $\mathbf{2 0}$ with some residual dicyclohexylurea which was later removed using size exclusion chromatography (BioRad Bio-Beads S-X2, 200-400 mesh, $\mathrm{CH}_{2} \mathrm{Cl}_{2}$, gravity flow) to afford $20(0.279 \mathrm{~g}, 68 \%)$ as a deep blue solid. $R_{\mathrm{f}}=0.17\left(\mathrm{CH}_{2} \mathrm{Cl}_{2}\right)$. UV-vis $\left(\mathrm{CH}_{2} \mathrm{Cl}_{2}\right) \lambda_{\max }(\varepsilon)$ : 271 (66 900), 309 (802 000), 330 (113 000), 440 (12 200), 551 (13 700), 594 (37 700), 645 (72 400) nm. IR (CDCl 3 , cast) 3048 (w), 2941 (s), 2863 (s), $2136(\mathrm{~m}), 1740(\mathrm{~s}) \mathrm{cm}^{-1} .{ }^{1} \mathrm{H}$ NMR (500 MHz, $\left.\mathrm{CDCl}_{3}\right): \delta 9.35$ (s, 4H), 9.31 (bs, 8H), 7.97-7.91 (m, 12H), 7.90-7.88 (m, 12H), 7.44-7.36 (m, 24H), $5.16(\mathrm{~s}, 8 \mathrm{H}), 4.79$ (s, $4 \mathrm{H}), 2.70(\mathrm{~s}, 8 \mathrm{H}), 1.60-1.46(\mathrm{~m}, 12 \mathrm{H}), 1.37(\mathrm{~d}, J=7.3 \mathrm{~Hz}, 12 \mathrm{H}), 1.36(\mathrm{~d}, J=7.3 \mathrm{~Hz}, 12 \mathrm{H})$, $1.35(\mathrm{~d}, J=7.2 \mathrm{~Hz}, 12 \mathrm{H}), 1.24(\mathrm{~d}, J=7.4 \mathrm{~Hz}, 12 \mathrm{H}), 1.22(\mathrm{~d}, J=7.3 \mathrm{~Hz}, 12 \mathrm{H}), 1.21(\mathrm{~d}, J=7.3$ $\mathrm{Hz}, 12 \mathrm{H}), 0.94$ (s, $18 \mathrm{H}), 0.10(\mathrm{~s}, 12 \mathrm{H}) .{ }^{13} \mathrm{C}$ NMR $\left(125 \mathrm{MHz}, \mathrm{CDCl}_{3}\right): \delta 172.1,142.9,137.0$, 135.6, 135.3, 133.5, 133.5, 132.4, 132.4, 131.4, 130.7, 130.7, 128.7, 128.6, 127.5, 126.4, 126.2, $126.2,125.5,118.5,118.2,118.1,105.9,105.9,105.8,105.6,105.3,105.2,66.5,64.9,29.2,26.0$, $18.4,18.3,18.2,18.2,18.1,12.1,12.1,-5.3$ (15 signals not observed). ${ }^{13} \mathrm{C}$ NMR (APT, 125 $\left.\mathrm{MHz}, \mathrm{CDCl}_{3}\right)$ : $\delta 172.1(\mathrm{C}), 142.9(\mathrm{C}), 137.0(\mathrm{C}), 135.6(\mathrm{CH}), 135.3(\mathrm{CH}), 133.5(\mathrm{C}), 133.5(\mathrm{C})$, $132.4(\mathrm{C}), 131.4(\mathrm{C}), 131.4(\mathrm{C}), 130.7(\mathrm{C}), 130.7(\mathrm{C}), 128.7(\mathrm{CH}), 128.6(\mathrm{CH}), 127.5(\mathrm{CH})$, $126.4(\mathrm{CH}), 126.2(\mathrm{CH}), 126.2(\mathrm{CH}), 125.5(\mathrm{CH}), 118.5(\mathrm{C}), 118.3(\mathrm{C}), 118.1(\mathrm{C}), 105.9(\mathrm{C})$, 
$105.9(\mathrm{C}), 105.8(\mathrm{C}), 105.6(\mathrm{C}), 105.3(\mathrm{C}), 105.2(\mathrm{C}), 66.5\left(\mathrm{CH}_{2}\right), 64.9\left(\mathrm{CH}_{2}\right), 29.2\left(\mathrm{CH}_{2}\right), 26.0$ $\left(\mathrm{CH}_{3}\right), 18.4(\mathrm{C}), 18.3\left(\mathrm{CH}_{3}\right), 18.2\left(\mathrm{CH}_{3}\right), 18.2\left(\mathrm{CH}_{3}\right), 18.2\left(\mathrm{CH}_{3}\right), 18.1\left(\mathrm{CH}_{3}\right), 12.1(\mathrm{CH}), 12.1$ $(\mathrm{CH}),-5.3\left(\mathrm{CH}_{3}\right)$ (14 signals not observed). MALDI $\left(\mathrm{CH}_{2} \mathrm{Cl}_{2}, \mathrm{DCTB}\right) \mathrm{m} / z 2693.3\left([\mathrm{M}+\mathrm{H}]^{+}\right.$, 100). TGA: $\mathrm{T}_{\mathrm{d}} \approx 380^{\circ} \mathrm{C}$. DSC: decomposition, $370{ }^{\circ} \mathrm{C}$ (onset) and $390^{\circ} \mathrm{C}$ (peak).

Synthesis of trimer 21.

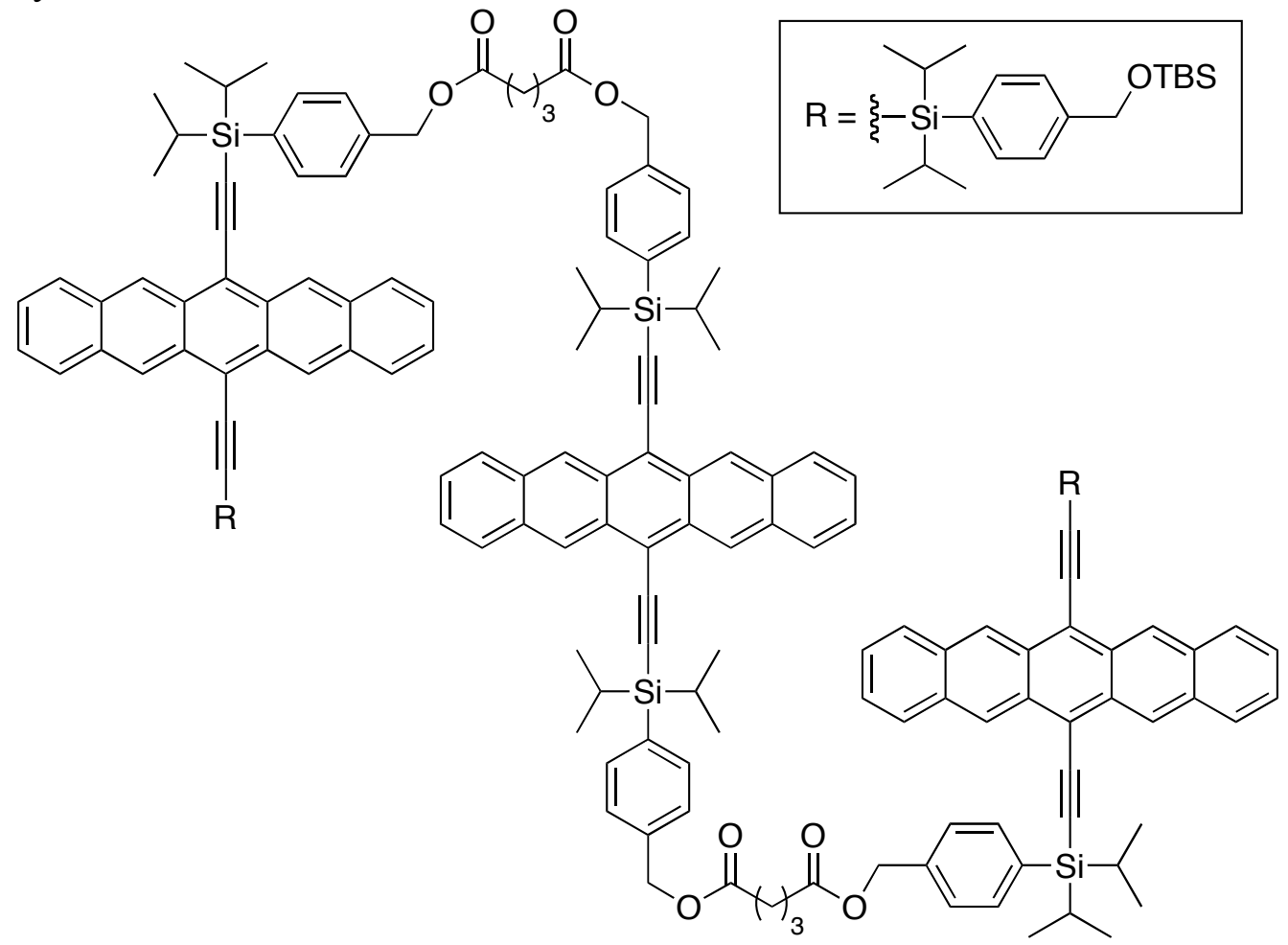

21

To a solution of $\mathbf{1 9}(0.184 \mathrm{~g}, 0.185 \mathrm{mmol})$ and $\mathbf{1}(0.0669 \mathrm{~g}, 0.0872 \mathrm{mmol})$ in dry $\mathrm{CH}_{2} \mathrm{Cl}_{2}(5 \mathrm{~mL})$ was added DMAP $(0.0710 \mathrm{~g}, 0.581 \mathrm{mmol})$ under $\mathrm{N}_{2}$ atmosphere. The reaction was allowed to stir at $\mathrm{rt}$ for $3 \mathrm{~h}$ before placing the flask in the fridge $\left(4^{\circ} \mathrm{C}\right)$ overnight to allow for most of the dicyclohexylurea to precipitate. The reaction was filtered and the filtrate was diluted with $\mathrm{CH}_{2} \mathrm{Cl}_{2}(80 \mathrm{~mL})$ and satd. aq. $\mathrm{NaHCO}_{3}(200 \mathrm{~mL})$. The mixture was separated and the organic phase was washed with satd. aq. $\mathrm{NaHCO}_{3}(200 \mathrm{~mL}), 5 \%$ aq. $\mathrm{NaHCO}_{3}(2 \times 200 \mathrm{~mL})$, satd. aq. $\mathrm{NaCl}(250 \mathrm{~mL})$, dried $\left(\mathrm{MgSO}_{4}\right)$, and the solvent removed in vacuo. Column chromatography (silica gel, $\mathrm{CH}_{2} \mathrm{Cl}_{2}$ ) afforded 21 with some residual dicyclohexylurea which was later removed using size exclusion chromatography (BioRad Bio-Beads S-X2, 200-400 mesh, $\mathrm{CH}_{2} \mathrm{Cl}_{2}$, gravity flow) to afford $21(0.142 \mathrm{~g}, 60 \%)$ as a deep blue solid. $R_{\mathrm{f}}=0.24\left(\mathrm{CH}_{2} \mathrm{Cl}_{2}\right)$. UV-vis $\left(\mathrm{CH}_{2} \mathrm{Cl}_{2}\right) \lambda_{\max }$ (ع): 271 (62 400), 309 (803 000), 330 (111 000), 440 (11 700), 551 (13 400), 594 (37 000), 645 (71 400) nm. IR (CDCl , cast) 3048 (w), 2943 (s), 2863 (s), 2136 (m), $1739(\mathrm{~s}) \mathrm{cm}^{-1} .{ }^{1} \mathrm{H}$ NMR $\left(500 \mathrm{MHz}, \mathrm{CDCl}_{3}\right): \delta 9.41(\mathrm{~s}, 4 \mathrm{H}), 9.38(2 \mathrm{x} \mathrm{s}, 8 \mathrm{H}), 8.03-7.97(\mathrm{~m}, 12 \mathrm{H}), 7.93(\mathrm{~m}, 12 \mathrm{H}), 7.59$ $7.48(\mathrm{~m}, 24 \mathrm{H}), 5.19(\mathrm{~s}, 8 \mathrm{H}), 4.83(\mathrm{~s}, 4 \mathrm{H}), 2.50(\mathrm{t}, J=7.4 \mathrm{~Hz}, 8 \mathrm{H}), 2.07$ (quintet, $J=7.3 \mathrm{~Hz}, 4 \mathrm{H}$ ), 1.59 (septet, $J=7.4 \mathrm{~Hz}, 4 \mathrm{H}$ ), 1.56 (septet, $J=7.3 \mathrm{~Hz}, 4 \mathrm{H}$ ), 1.56 (septet, $J=7.1 \mathrm{~Hz}, 4 \mathrm{H}$ ), 1.43 (d, $J=7.2 \mathrm{~Hz}, 12 \mathrm{H}), 1.41(\mathrm{~d}, J=7.3 \mathrm{~Hz}, 12 \mathrm{H}), 1.41(\mathrm{~d}, J=7.3 \mathrm{~Hz}, 12 \mathrm{H}), 1.30(\mathrm{~d}, J=7.4 \mathrm{~Hz}, 12 \mathrm{H})$, $1.28(\mathrm{~d}, J=7.4 \mathrm{~Hz}, 12 \mathrm{H}), 1.27(\mathrm{~d}, J=7.3 \mathrm{~Hz}, 12 \mathrm{H}), 0.98(\mathrm{~s}, 18 \mathrm{H}), 0.15(\mathrm{~s}, 12 \mathrm{H}) \cdot{ }^{13} \mathrm{C}$ NMR $(125$ $\mathrm{MHz}, \mathrm{CDCl}_{3}$ ): $\delta 172.7,142.9,137.2,137.2,135.6,135.6,135.3,133.4,133.4,132.4,132.4$, $132.4,131.4,130.7,130.7,128.7,128.6,127.5,126.4,126.3,126.2,126.2,126.2,125.5,118.5$, 
118.3, 118.1, 105.9, 105.9, 105.8, 105.6, 105.3, 105.2, 66.2, 64.9, 33.3, 26.0, 20.1, 18.4, 18.2, 18.2, 18.2, 18.1, 12.1, 12.1, -5.3 (10 signals not observed). ${ }^{13} \mathrm{C}$ NMR (APT, $125 \mathrm{MHz}, \mathrm{CDCl}_{3}$ ): ठ $172.7(\mathrm{C}), 142.9(\mathrm{C}), 137.2(\mathrm{C}), 137.2(\mathrm{C}), 135.6(\mathrm{CH}), 135.3(\mathrm{CH}), 133.4(\mathrm{C}), 133.4(\mathrm{C})$, $132.4(\mathrm{C}), 132.4(\mathrm{C}), 131.4(\mathrm{C}), 130.7(\mathrm{C}), 130.7(\mathrm{C}), 128.7(\mathrm{CH}), 128.6(\mathrm{CH}), 127.5(\mathrm{CH})$, $126.4(\mathrm{CH}), 126.3(\mathrm{CH}), 126.2(\mathrm{CH}), 126.2(\mathrm{CH}), 126.2(\mathrm{CH}), 125.5(\mathrm{CH}), 118.5(\mathrm{C}), 118.3(\mathrm{C})$, 118.1 (C), 105.9 (C), $105.9(\mathrm{C}), 105.8(\mathrm{C}), 105.6(\mathrm{C}), 105.3(\mathrm{C}), 105.2(\mathrm{C}), 66.2\left(\mathrm{CH}_{2}\right), 64.9$ $\left(\mathrm{CH}_{2}\right), 33.3\left(\mathrm{CH}_{2}\right), 26.0\left(\mathrm{CH}_{3}\right), 20.1\left(\mathrm{CH}_{3}\right), 18.4(\mathrm{C}), 18.2\left(\mathrm{CH}_{3}\right), 18.2\left(\mathrm{CH}_{3}\right), 18.2\left(\mathrm{CH}_{3}\right), 18.1$ $\left(\mathrm{CH}_{3}\right), 12.1(\mathrm{CH}), 12.1(\mathrm{CH}),-5.3\left(\mathrm{CH}_{3}\right)$ (12 signals not observed). MALDI $\left(\mathrm{CH}_{2} \mathrm{Cl}_{2}, \mathrm{DCTB}\right)$ $m / z 2721.1\left([\mathrm{M}+\mathrm{H}]^{+}, 100\right)$. TGA: $\mathrm{T}_{\mathrm{d}} \approx 380{ }^{\circ} \mathrm{C}$. DSC: decomposition, $365^{\circ} \mathrm{C}$ (onset) and $390{ }^{\circ} \mathrm{C}$ (peak). 


\section{Discussion of MALDI Mass Spectrometry Results and Structures of Polymers 8 and 9}

From the polymerization reactions described above, the polymeric mixture can contain various structures including the four structures shown below (Figure S1). A macromolecule can be terminated with an alcohol at one end and a carboxylic acid at the other (structure A), or a carboxylic acid at both ends (structure B), or an alcohol at both ends (structure C), or the polymerization can cyclize onto itself resulting into a macrocyclic molecule (structure $\mathrm{D}$ ). The MALDI mass spectrometry spectra of polymers 8 and $\mathbf{9}$ are shown below (Figures S2 and S3, respectively), in which the signal corresponding to the various structures have been labeled using the legend below (Figure S1).
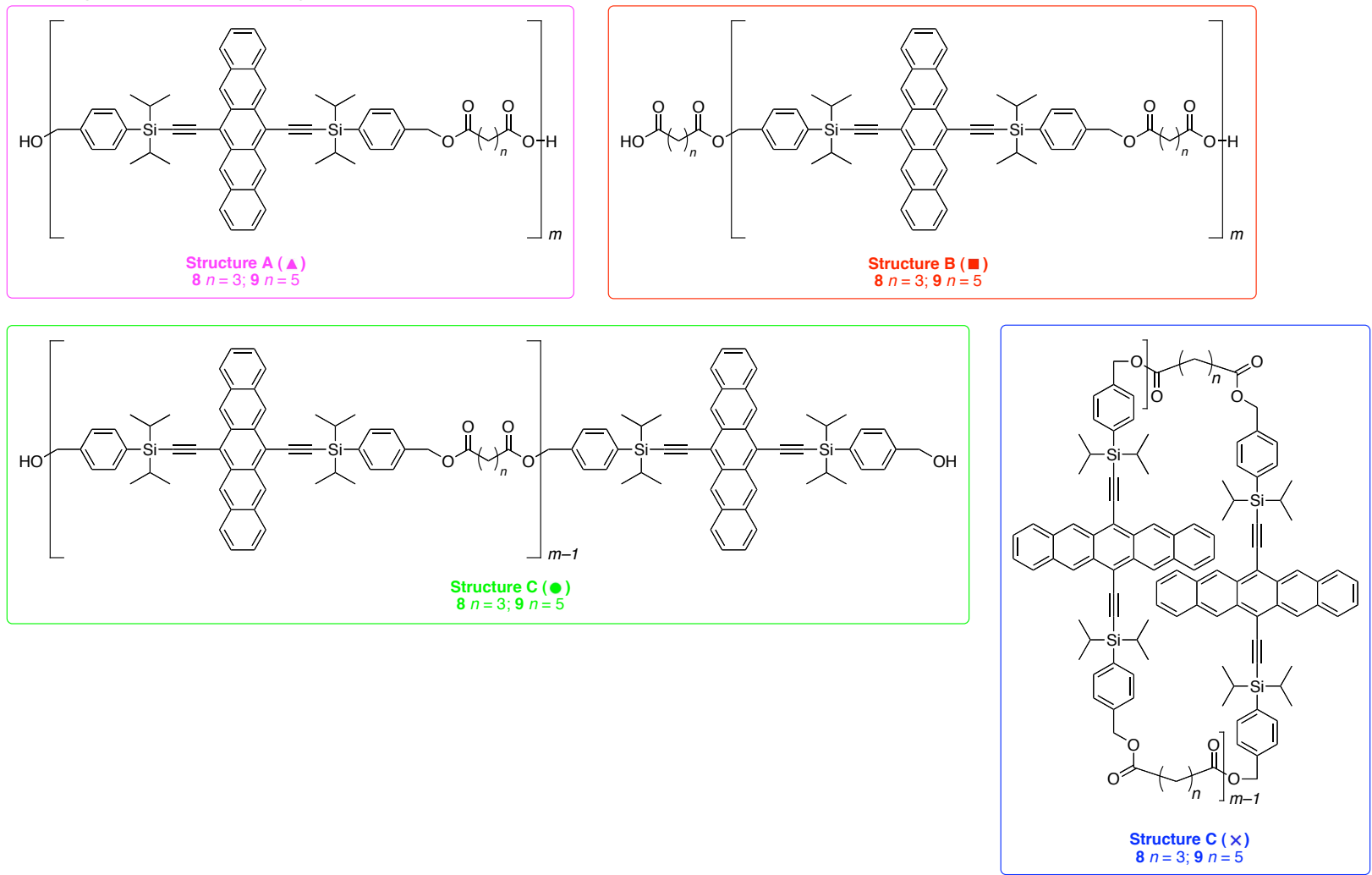

Figure S1. Legend for structures observed in the MALDI mass spectrometry. 


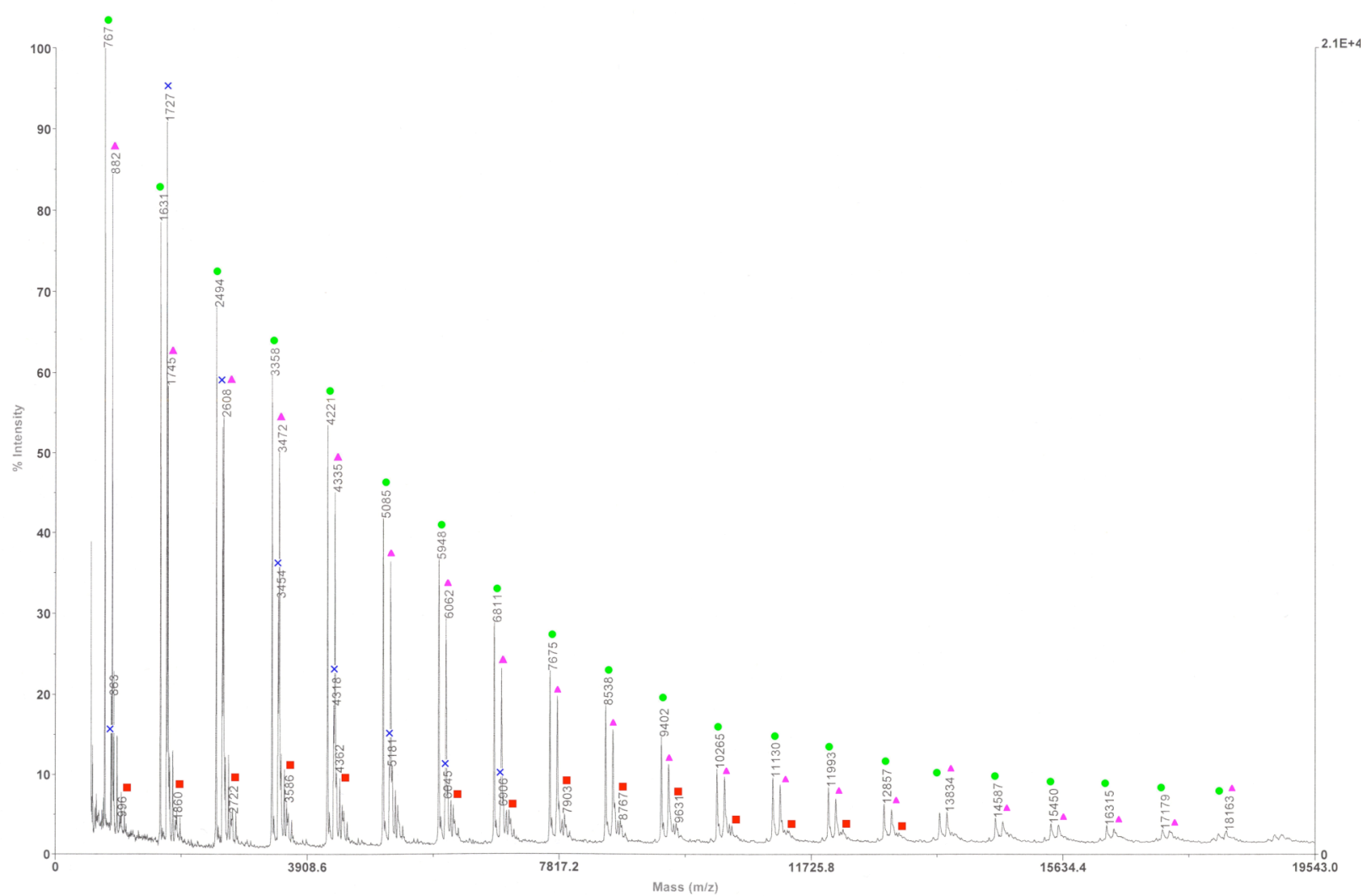

Figure S2: MALDI Mass Spectrometry Spectrum of Pentacene Polymer 8.

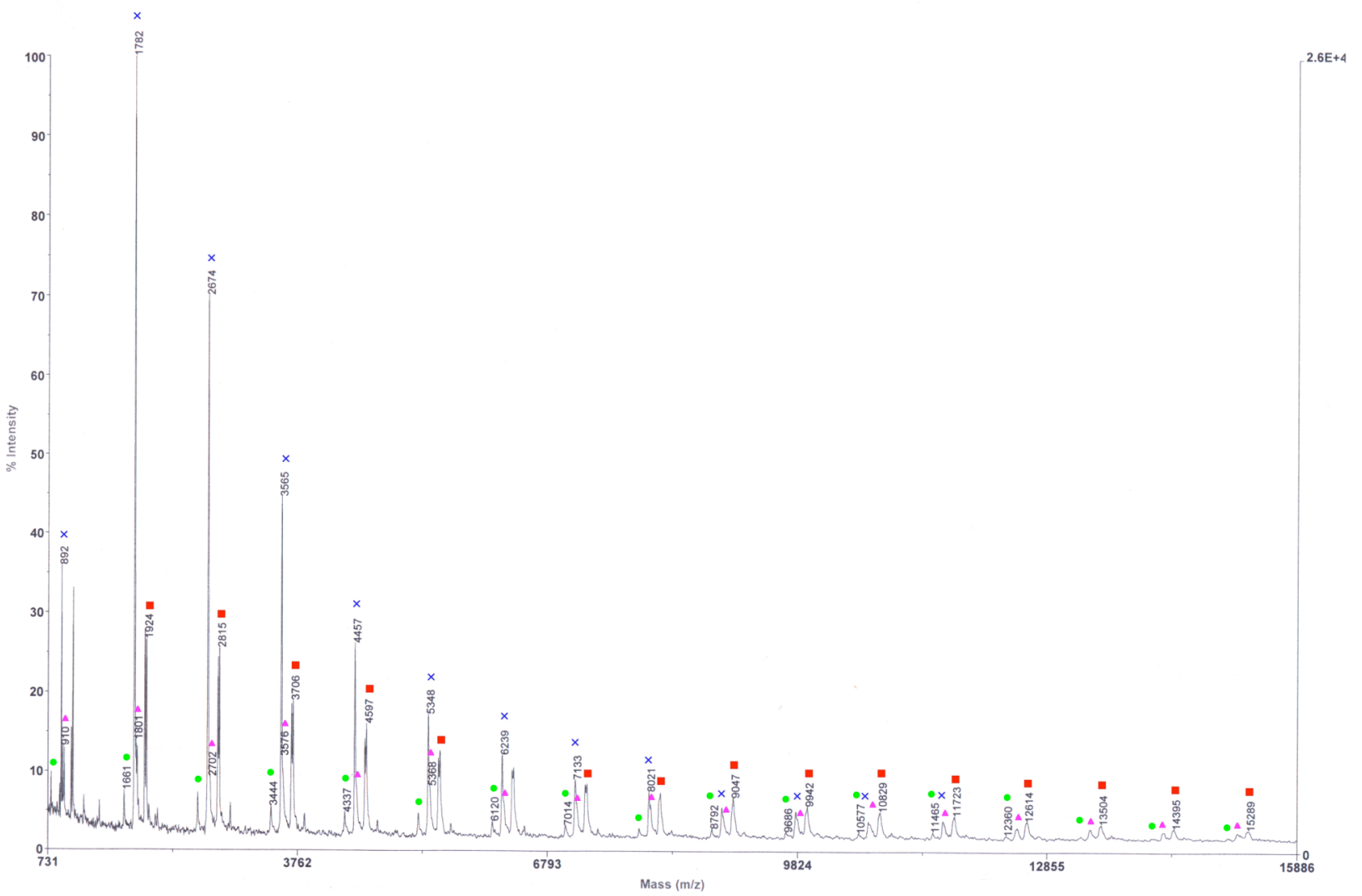

Figure S3: MALDI Mass Spectrometry Spectrum of Pentacene Polymer 9. 


\section{UV-Vis of Thin-Films of Pentacene Polymers 8 and 9}

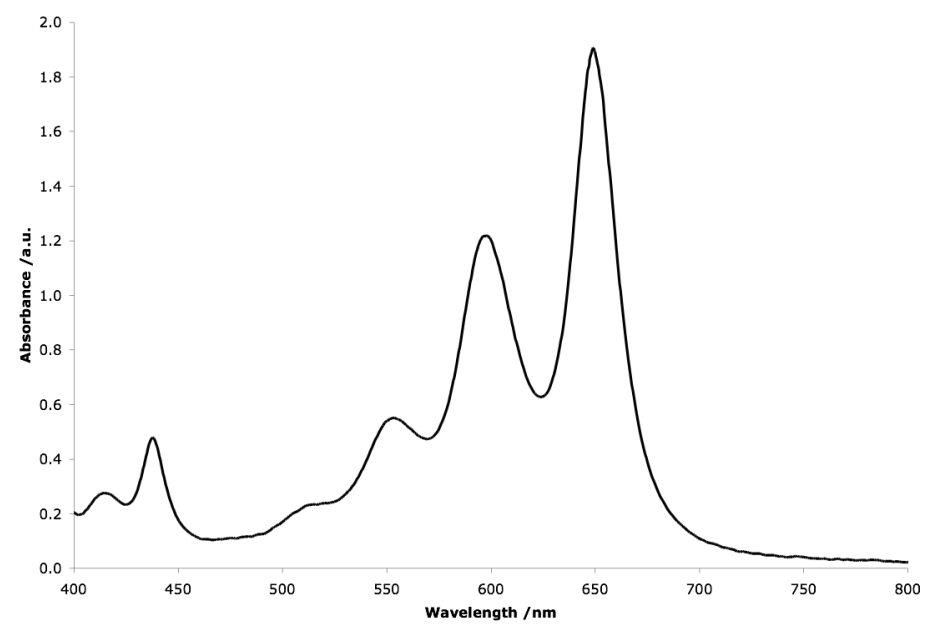

Figure S4: UV-vis of thin film of polymer 8 (cast from $\mathrm{CH}_{2} \mathrm{Cl}_{2}$ ).

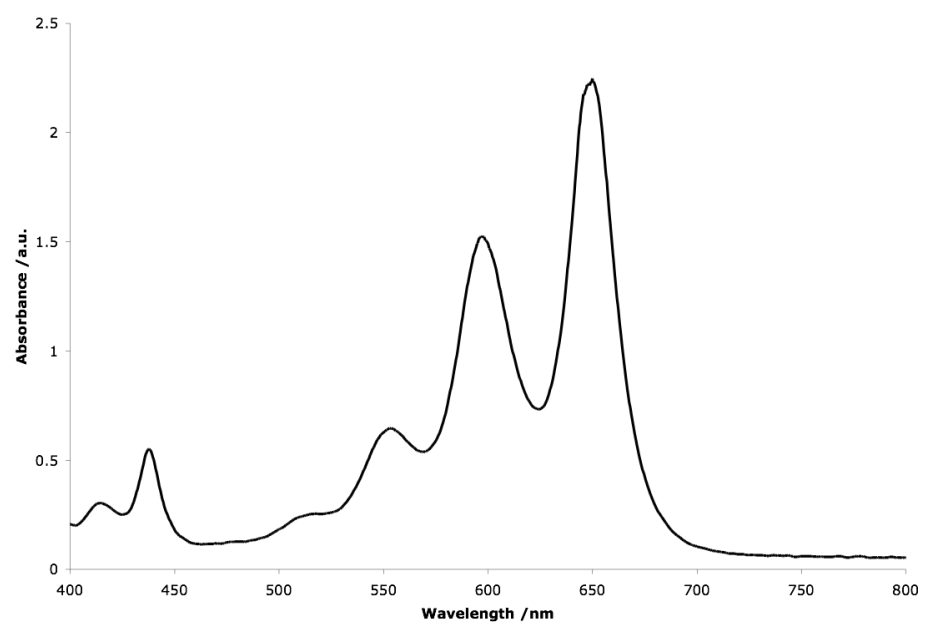

Figure S5: UV-vis of thin film of polymer 9 (cast from $\mathrm{CH}_{2} \mathrm{Cl}_{2}$ ). 


\section{${ }^{1} \mathrm{H}$ and ${ }^{13} \mathrm{C}$ NMR of Compounds 1-7}

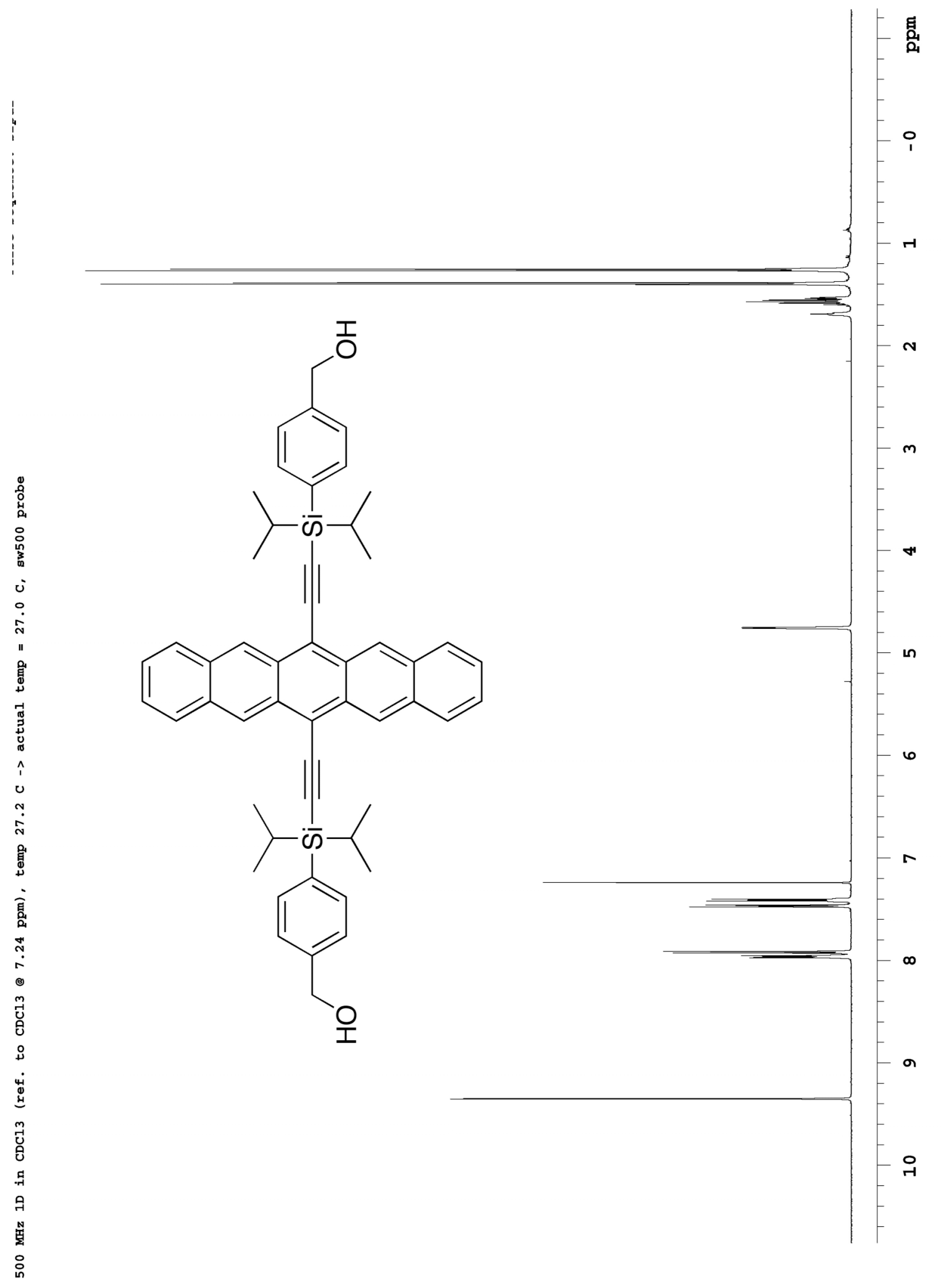

Figure S6: ${ }^{1} \mathrm{H}$ NMR spectrum of compound $\mathbf{1}$. 


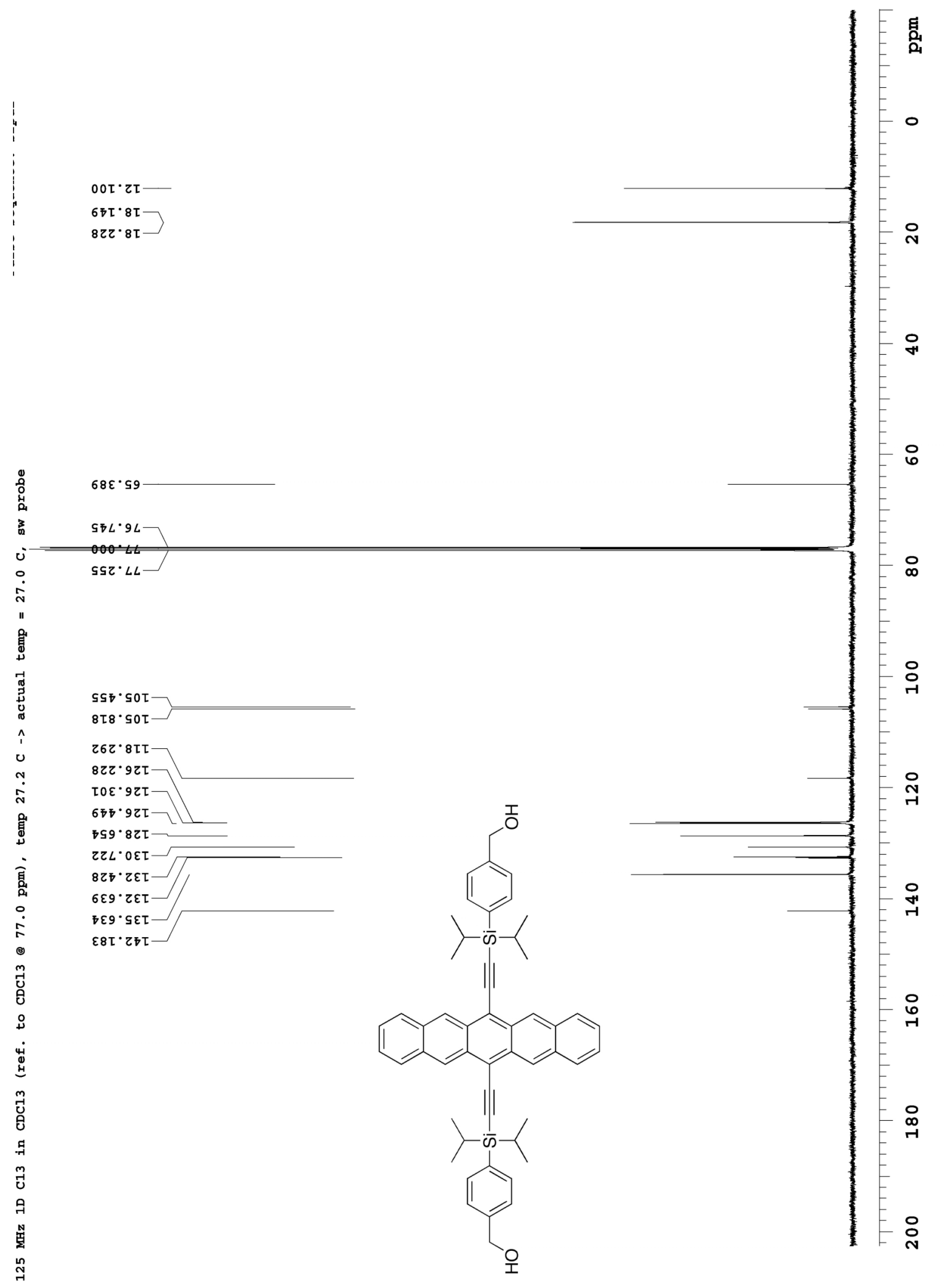

Figure S7: ${ }^{13} \mathrm{C}$ NMR spectrum of compound $\mathbf{1}$. 


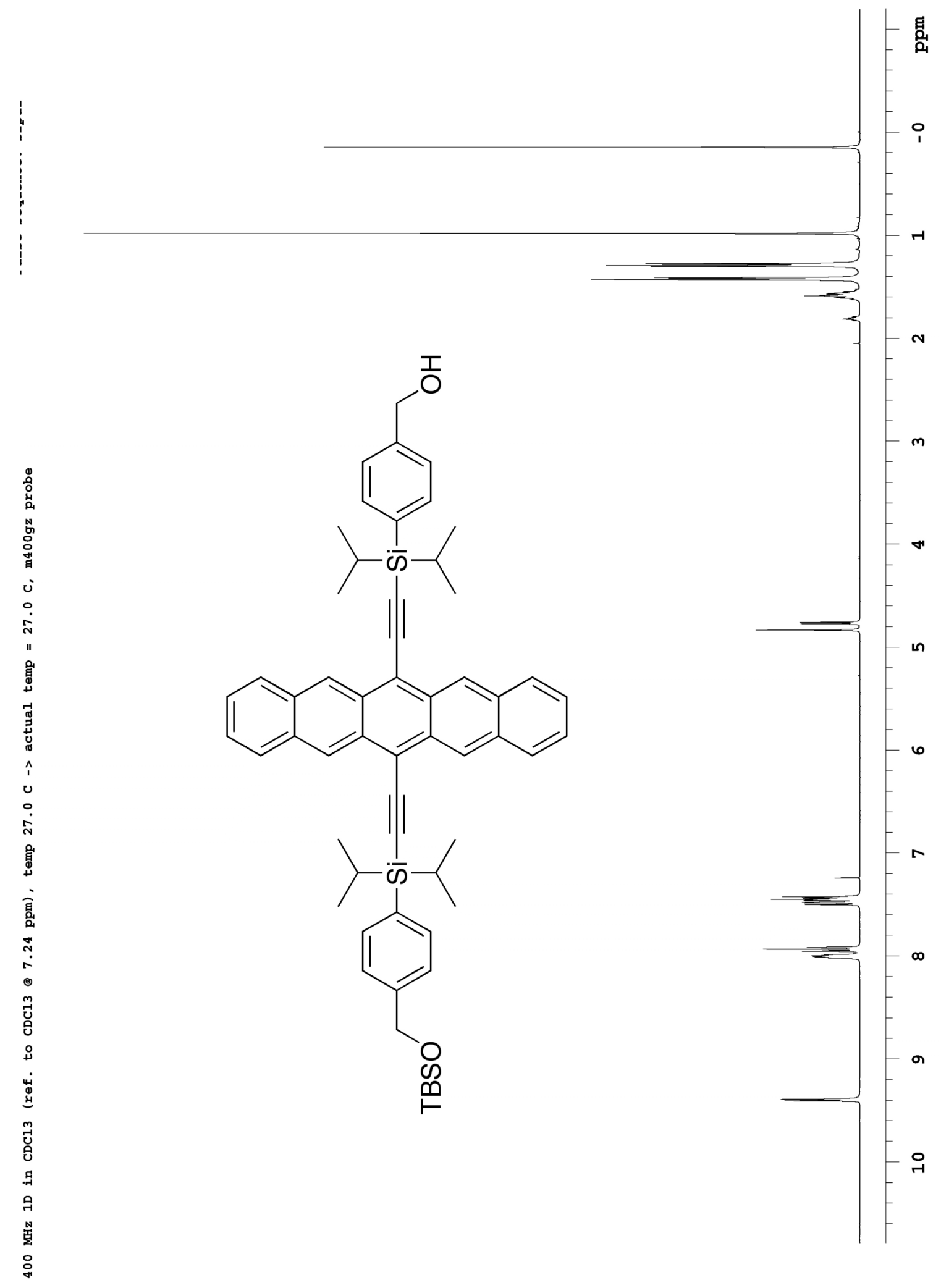

Figure S8: ${ }^{1} \mathrm{H}$ NMR spectrum of compound 2. 


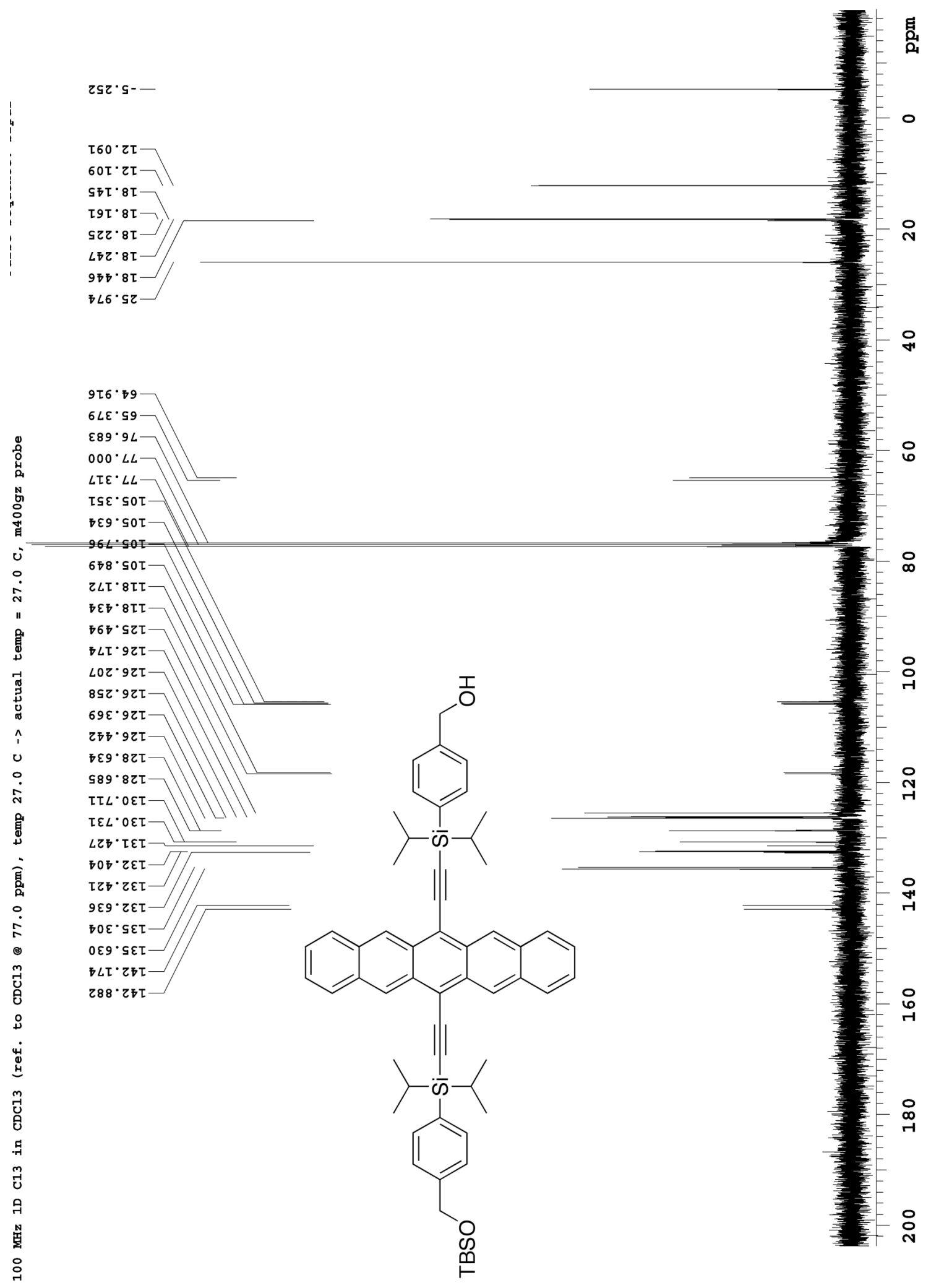

Figure S9: ${ }^{13} \mathrm{C}$ NMR spectrum of compound 2. 


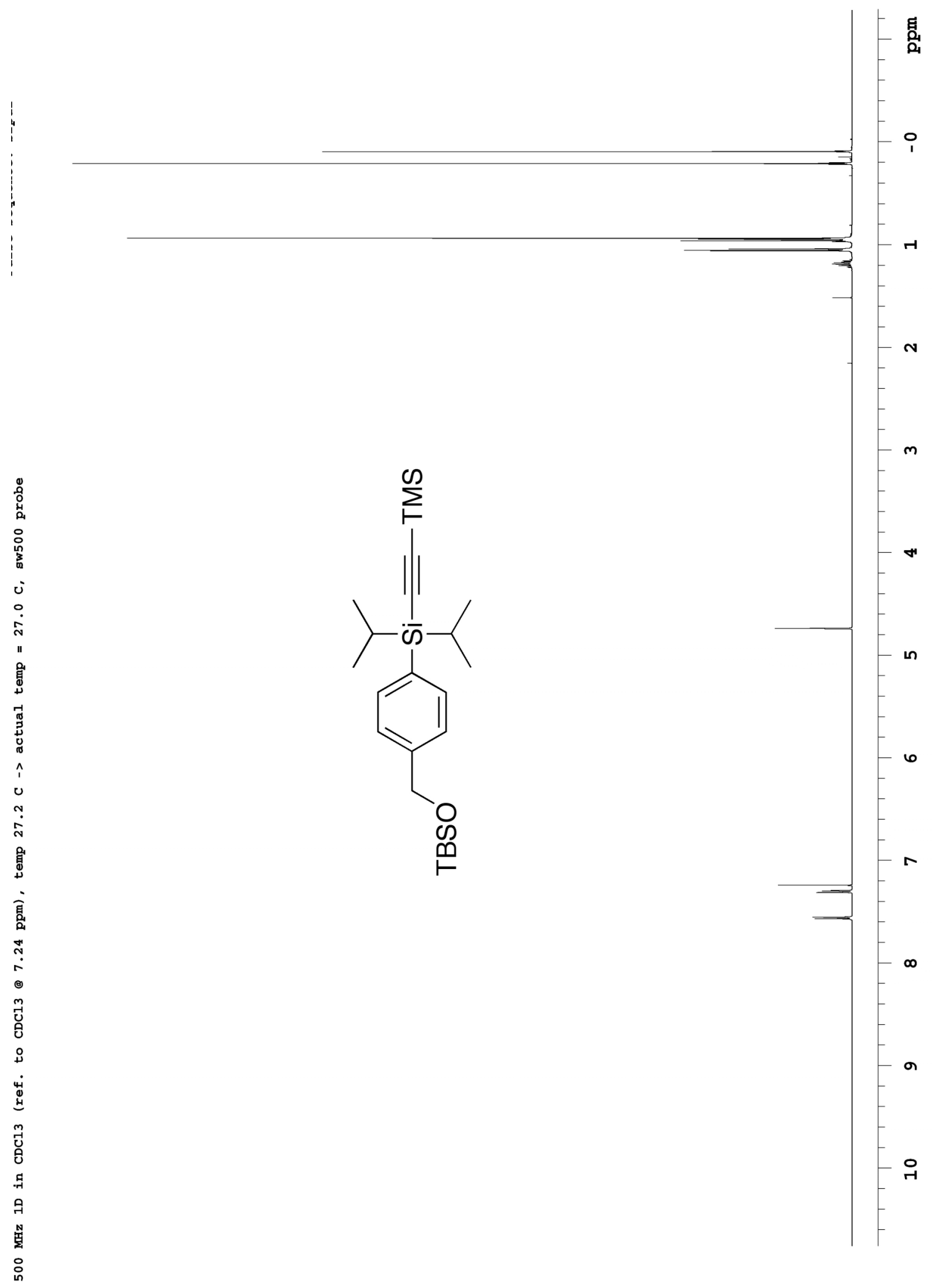

Figure S10: ${ }^{1} \mathrm{H}$ NMR spectrum of compound 4. 


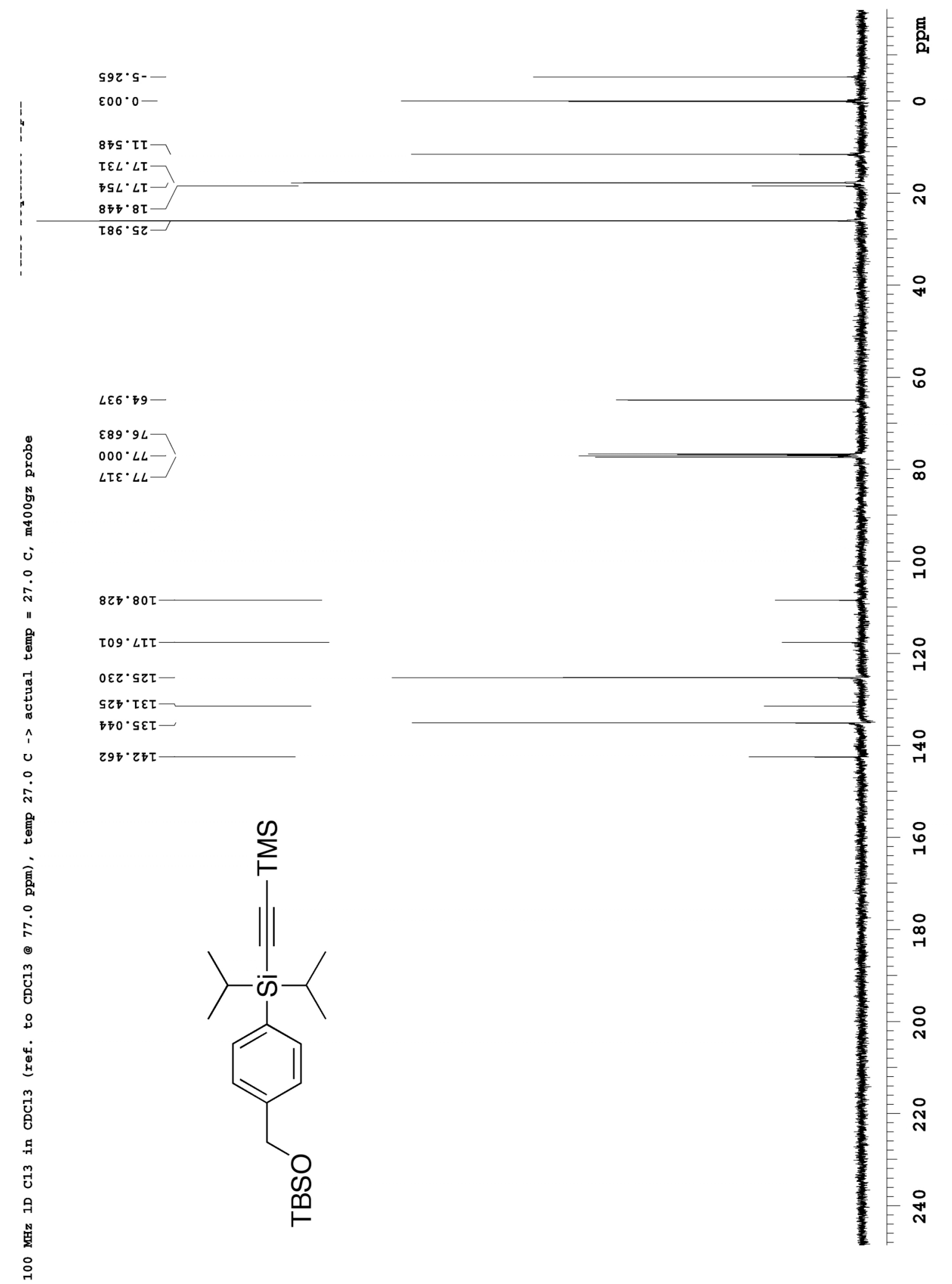

Figure S11: ${ }^{13} \mathrm{C}$ NMR spectrum of compound 4. 


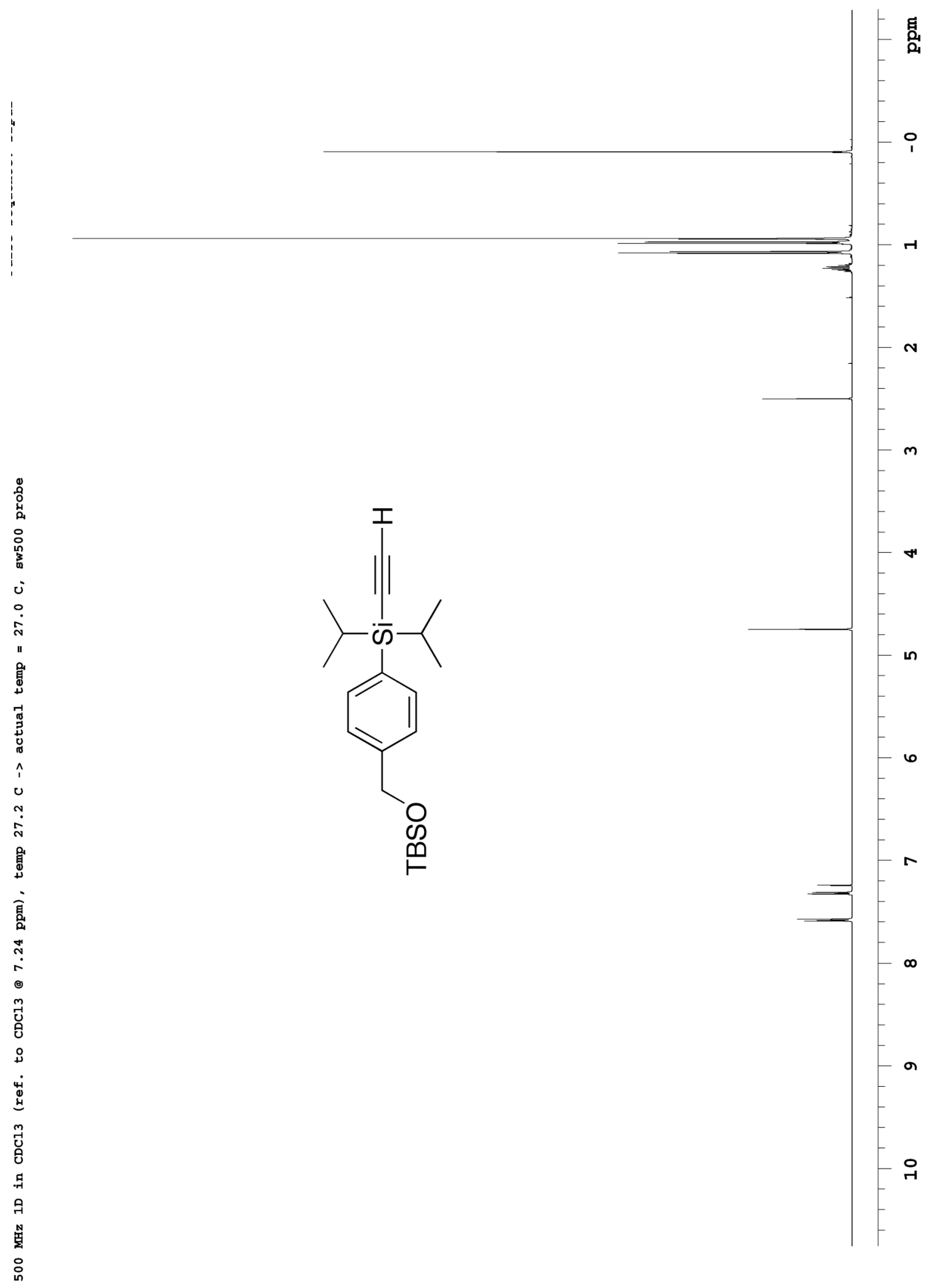

Figure S12: ${ }^{1} \mathrm{H}$ NMR spectrum of compound 5. 


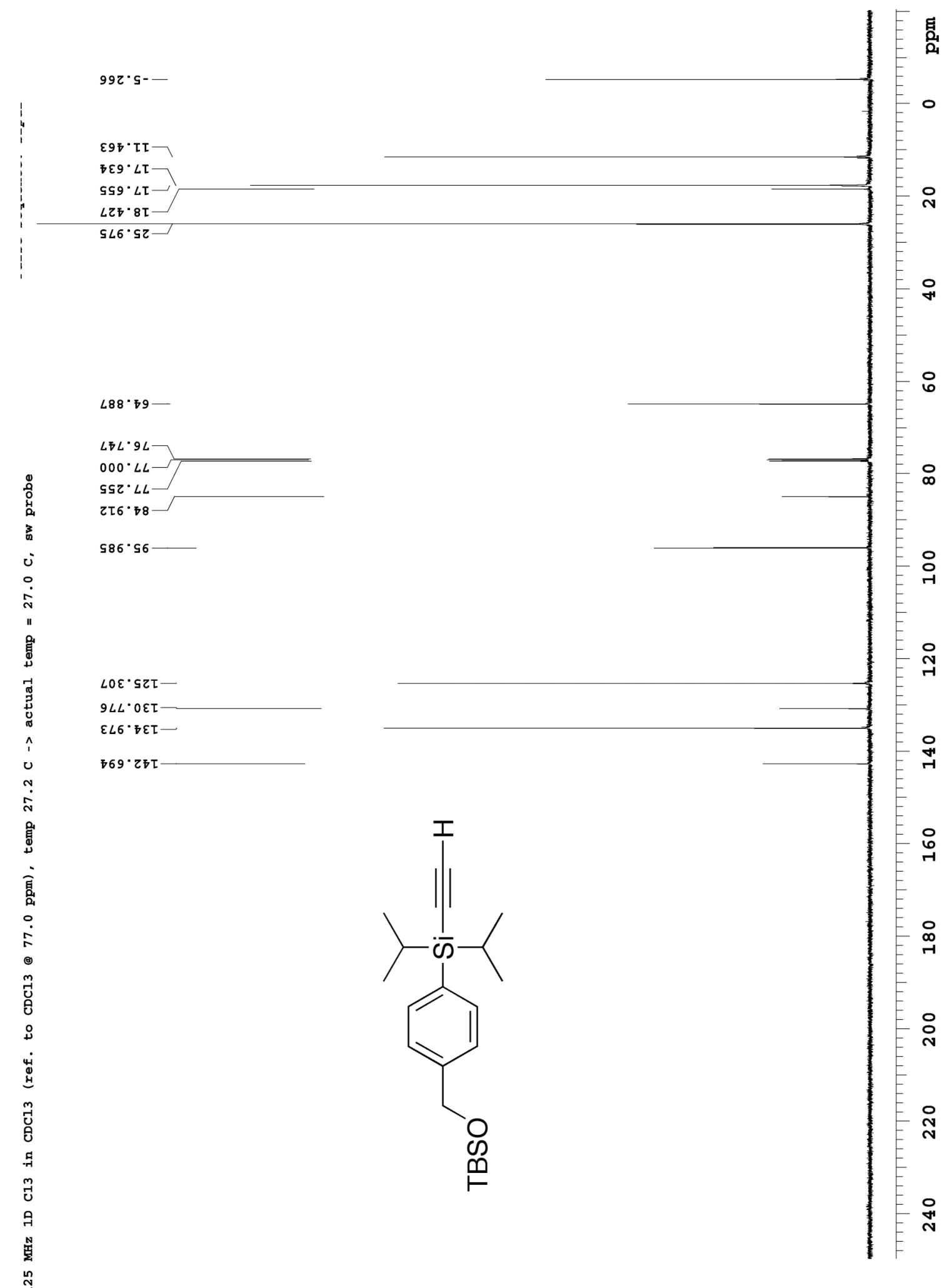

Figure S13: ${ }^{13} \mathrm{C}$ NMR spectrum of compound 5. 


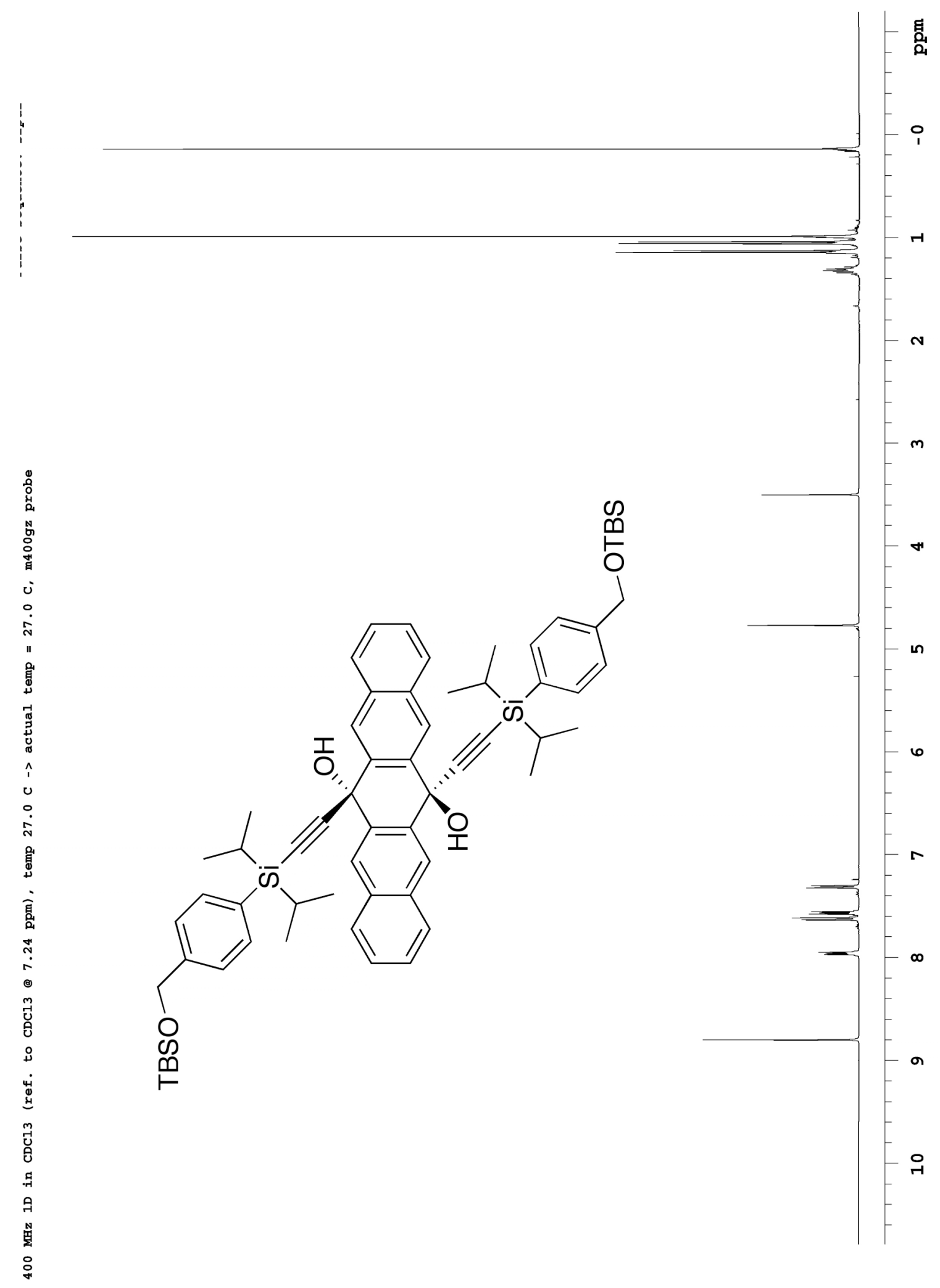

Figure S14: ${ }^{1} \mathrm{H}$ NMR spectrum of compound trans-6. 


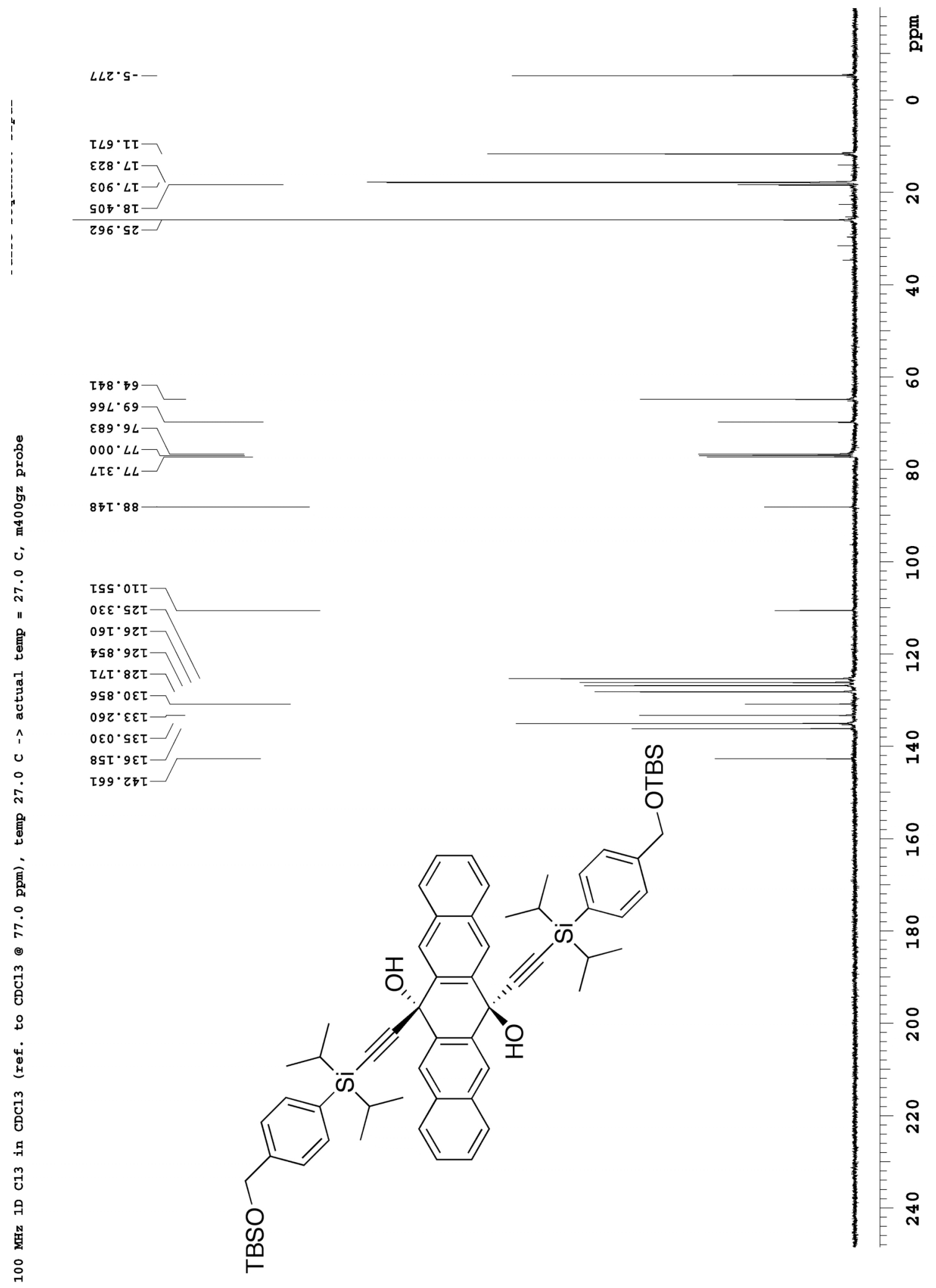

Figure S15: ${ }^{13} \mathrm{C}$ NMR spectrum of compound trans-6. 


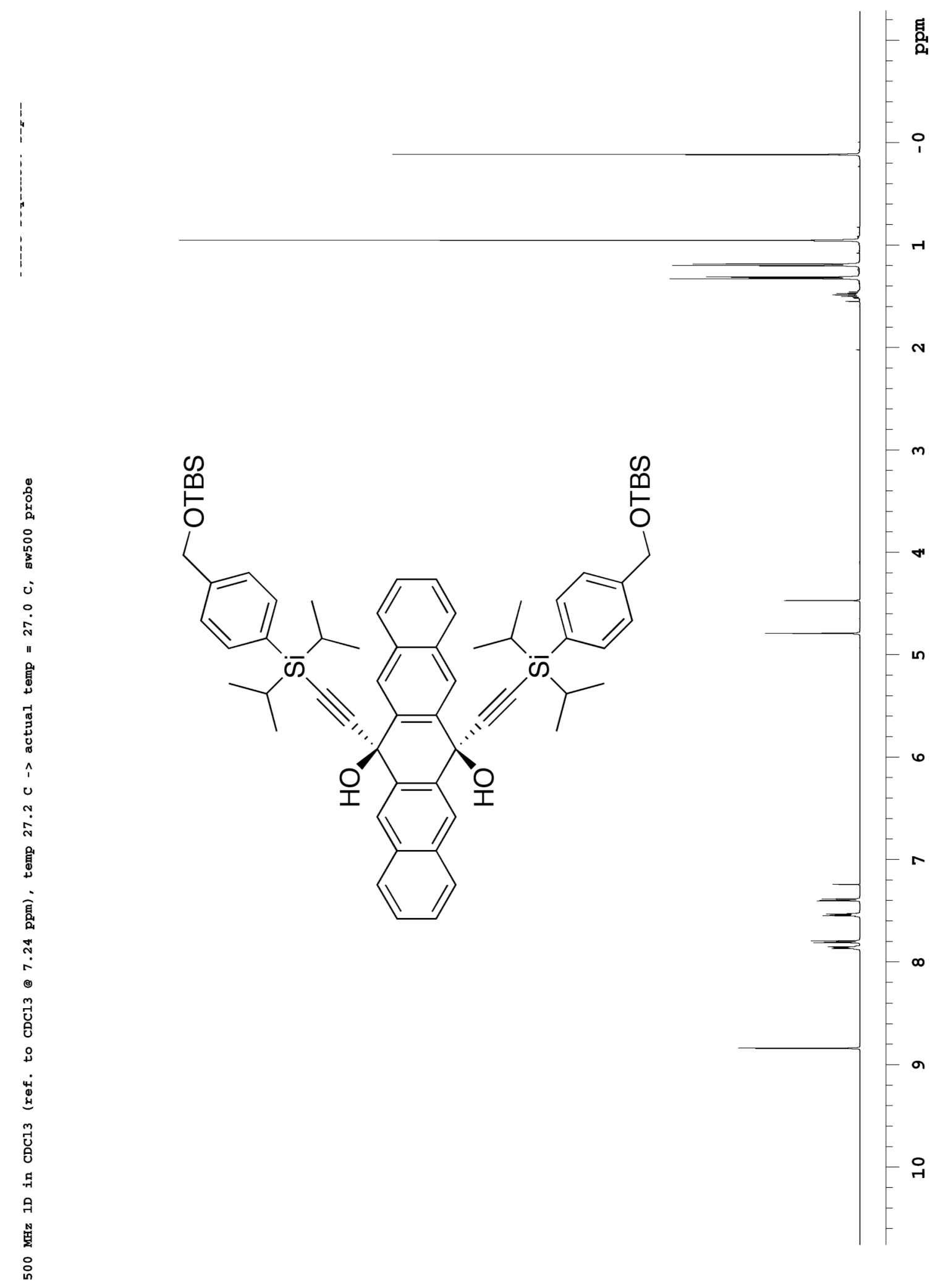

Figure S16: ${ }^{1} \mathrm{H}$ NMR spectrum of compound cis-6. 


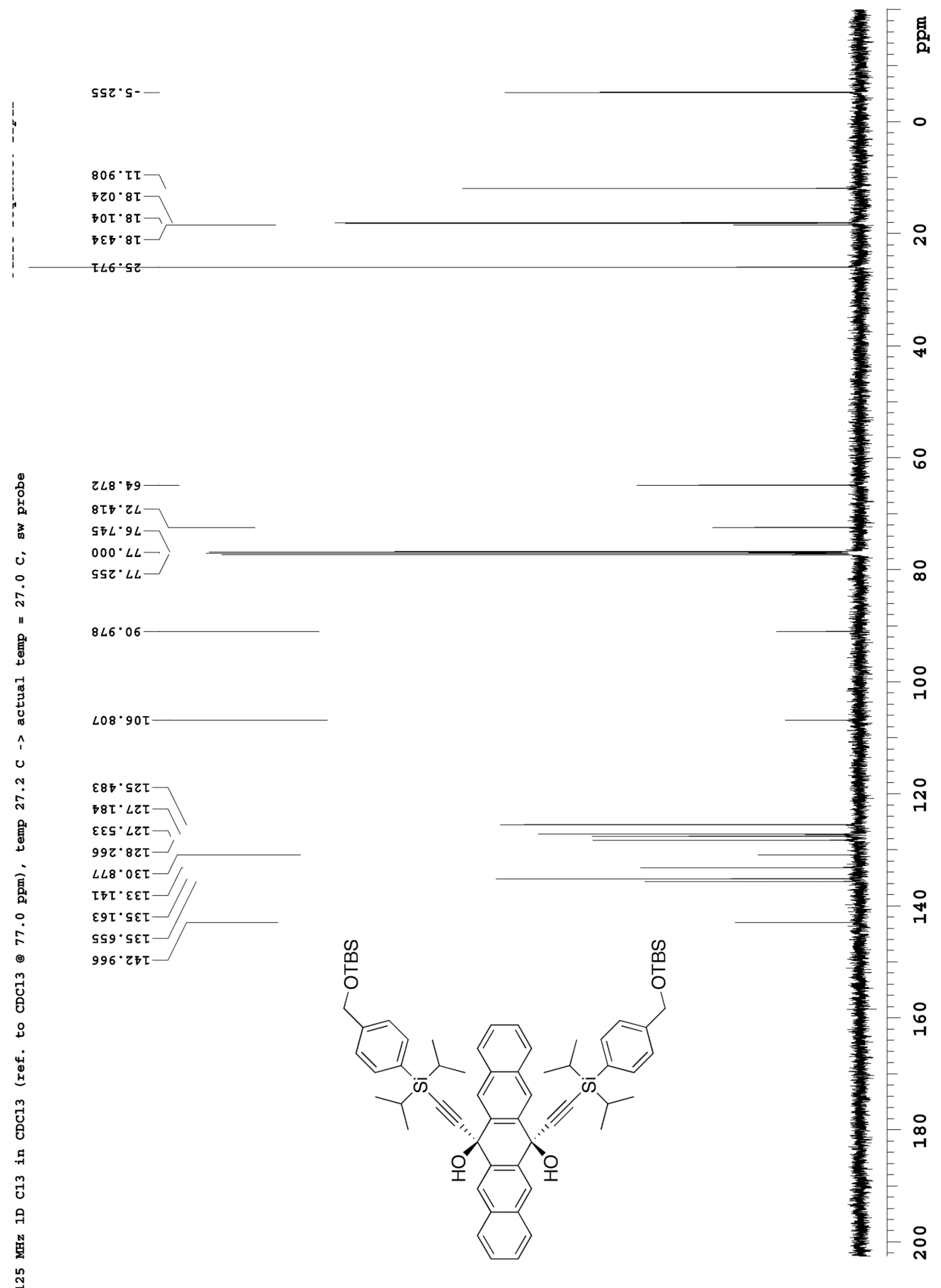

Figure S17: ${ }^{13} \mathrm{C}$ NMR spectrum of compound cis-6. 


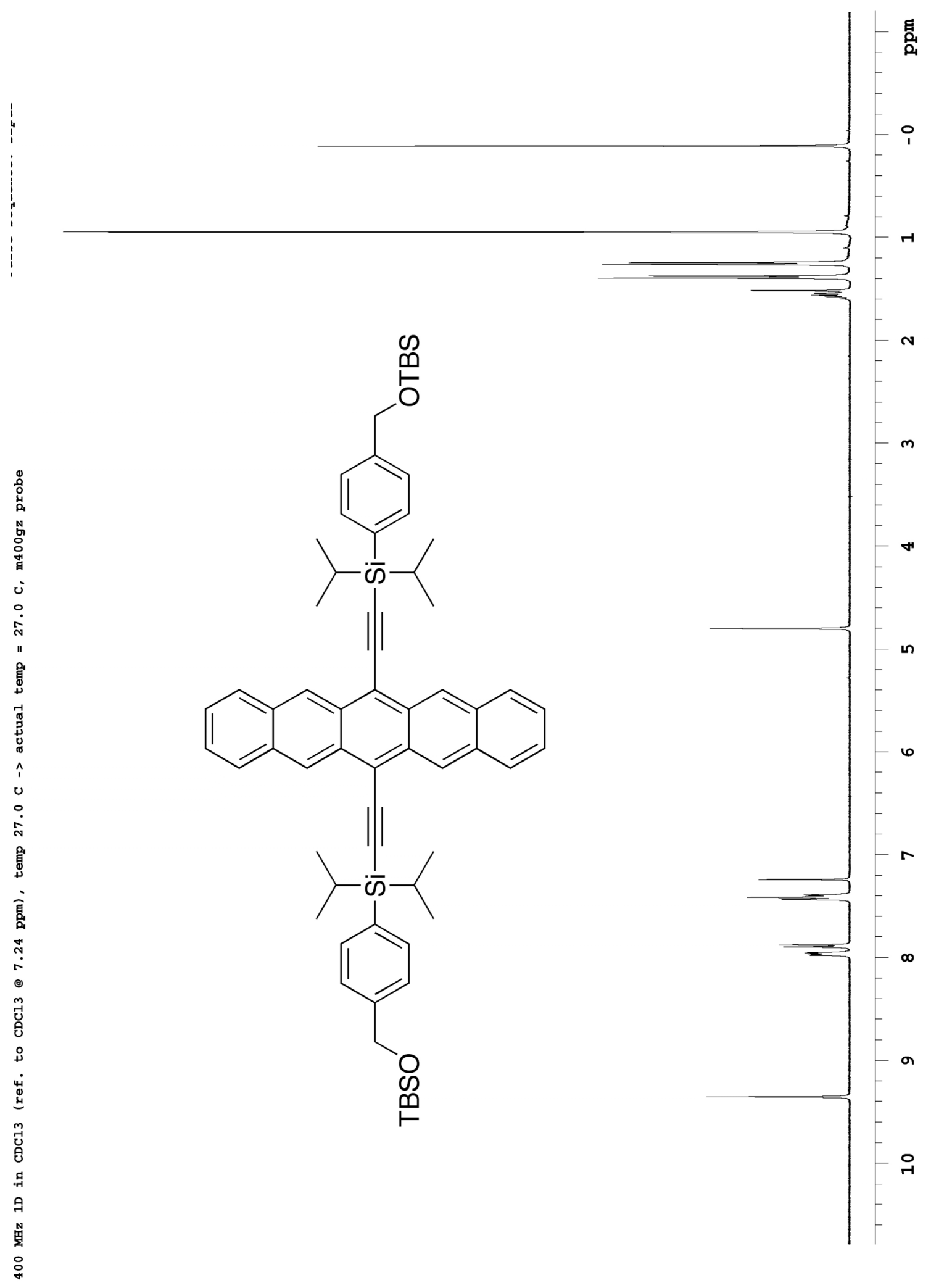

Figure S18: ${ }^{1} \mathrm{H}$ NMR spectrum of compound 7. 


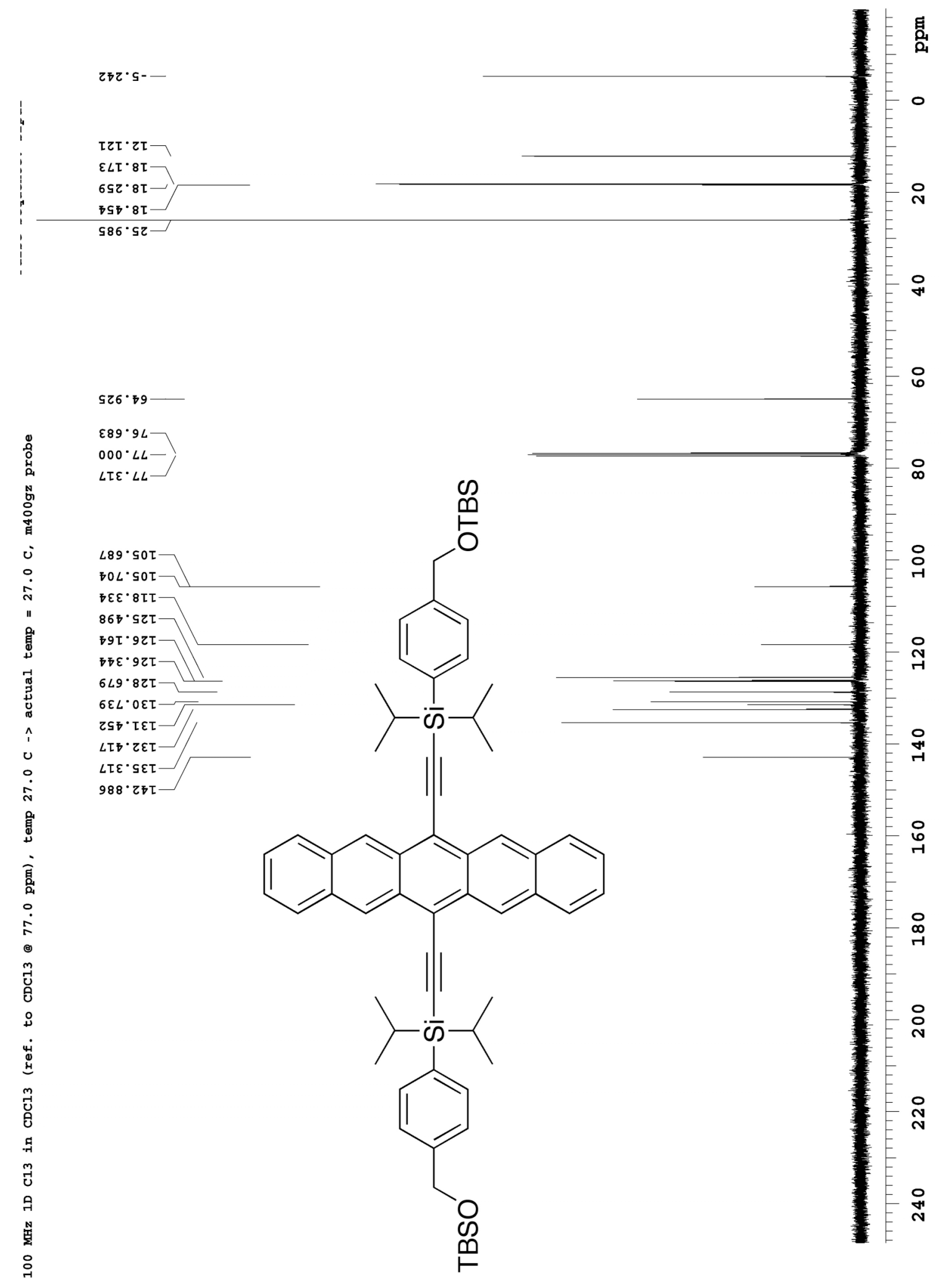

Figure S19: ${ }^{13} \mathrm{C}$ NMR spectrum of compound 7. 